\title{
TAP FREE ENERGY, SPIN GLASSES AND VARIATIONAL INFERENCE
}

\author{
By ZHOU FAN ${ }^{1}$, SONG MEI ${ }^{2}$ AND ANDREA MONTANARI ${ }^{3}$ \\ ${ }^{1}$ Department of Statistics and Data Science, Yale University, zhou.fan@yale.edu \\ ${ }^{2}$ Department of Statistics, University of California, Berkeley, songmei@berkeley.edu \\ ${ }^{3}$ Department of Statistics and Department of Electrical Engineering, Stanford University, montanari@stanford.edu
}

We consider the Sherrington-Kirkpatrick model of spin glasses with ferromagnetically biased couplings. For a specific choice of the couplings mean, the resulting Gibbs measure is equivalent to the Bayesian posterior for a highdimensional estimation problem known as " $\mathbb{Z}_{2}$ synchronization." Statistical physics suggests to compute the expectation with respect to this Gibbs measure (the posterior mean in the synchronization problem), by minimizing the so-called Thouless-Anderson-Palmer (TAP) free energy, instead of the mean field (MF) free energy. We prove that this identification is correct, provided the ferromagnetic bias is larger than a constant (i.e., the noise level is small enough in synchronization). Namely, we prove that the scaled $\ell_{2}$ distance between any low energy local minimizers of the TAP free energy and the mean of the Gibbs measure vanishes in the large size limit. Our proof technique is based on upper bounding the expected number of critical points of the TAP free energy using the Kac-Rice formula.

1. Introduction and main results. Computing expectations of a high-dimensional probability distribution is a central problem in computer science, statistics and statistical physics. While a number of mathematical and computational techniques have been developed for this task, fundamental questions remain unanswered even for seemingly simple models.

Consider the problem of estimating a vector $\boldsymbol{x} \in\{+1,-1\}^{n}$ from the observation $\boldsymbol{Y} \in \mathbb{R}^{n \times n}$ given by

$$
\boldsymbol{Y}=\frac{\lambda}{n} \boldsymbol{x} \boldsymbol{x}^{\top}+\boldsymbol{W} .
$$

Here, $\boldsymbol{W}$ is an unknown noise matrix which is distributed according to the Gaussian orthogonal ensemble (GOE). Namely, $\boldsymbol{W}=\boldsymbol{W}^{\top} \in \mathbb{R}^{n \times n}$ where $\left(W_{i j}\right)_{1 \leq i \leq j \leq n}$ are independent centered Gaussian random variables with off-diagonal entries having variance $1 / n$ and diagonal entries having variance $2 / n$. (In what follows, we will write $\boldsymbol{W} \sim \operatorname{GOE}(n)$.) The parameter $\lambda \in(0, \infty)$ is assumed to be known and corresponds to a signal-to-noise ratio.

This problem is known as $\mathbb{Z}_{2}$ synchronization [61] and is closely related to correlation clustering [10]. It has applications to determining the orientation of a manifold [62] and as a simplified model for the two-groups stochastic block model [39] and for topic models $[15,37] . \mathbb{Z}_{2}$ synchronization is known to undergo a phase transition at $\lambda=1$ : for $\lambda<1$, no estimator can achieve a correlation with the true signal $\boldsymbol{x}$ which is bounded away from zero. Viceversa, for $\lambda>1$, there exist estimators that achieve a strictly positive correlation; see, for example, $[32,49]$.

In this paper we consider the regime $\lambda>1$ and study the optimal estimator. Notice thateven in absence of noise - the vector $\boldsymbol{x}$ can be determined from the observation $\boldsymbol{Y}$ only up to a sign. In order to resolve this ambiguity, we set as our goal to estimate the $n \times n$ matrix of

Received June 2019; revised March 2020.

MSC2020 subject classifications. 60F10.

Key words and phrases. Bayesian inference, TAP complexity, Sherrington-Kirkpatrick model, Kac-Rice formula, free probability. 
relative signs $\boldsymbol{X}=\boldsymbol{x} \boldsymbol{x}^{\top}$. An estimator will be a map $\widehat{\boldsymbol{X}}: \mathbb{R}^{n \times n} \rightarrow \mathbb{R}^{n \times n}, \boldsymbol{Y} \mapsto \widehat{\boldsymbol{X}}(\boldsymbol{Y})$. We will see that the optimal $\widehat{\boldsymbol{X}}$ is approximately of rank one, that is, $\widehat{\boldsymbol{X}}(\boldsymbol{Y}) \approx \boldsymbol{m}_{\star} \boldsymbol{m}_{\star}^{\top}$, and, therefore, $\boldsymbol{m}_{\star} \in \mathbb{R}^{n}$ can be viewed as an estimator of $\pm \boldsymbol{x}$.

By the symmetry of the problem, it is reasonable to consider Bayes estimation with respect to the uniform prior. ${ }^{1}$ Namely, we assume $\boldsymbol{x} \sim \operatorname{Unif}\left(\{+1,-1\}^{n}\right)$ and try to minimize the matrix mean square error, defined by

$$
\operatorname{MMSE}_{n}(\lambda)=\inf _{\widehat{\boldsymbol{X}}} \frac{1}{n^{2}} \mathbb{E}\left[\left\|\widehat{\boldsymbol{X}}(\boldsymbol{Y})-\boldsymbol{x} \boldsymbol{x}^{\top}\right\|_{F}^{2}\right] .
$$

The minimum of $\operatorname{MMSE}_{n}(\lambda)$ is achieved by the posterior mean ${ }^{2}$

$$
\widehat{\boldsymbol{X}}_{\text {Bayes }}=\widehat{\boldsymbol{X}}_{\text {Bayes }}(\boldsymbol{Y})=\mathbb{E}\left[\boldsymbol{x} \boldsymbol{x}^{\top} \mid \boldsymbol{Y}\right] .
$$

The asymptotics of the Bayes risk $\lim _{n \rightarrow \infty} \operatorname{MMSE}_{n}(\lambda)$ was calculated in [32].

Computing the posterior expectation requires summing functions over $\boldsymbol{x} \in\{+1,-1\}^{n}$, an example of the high-dimensional integration problem mentioned above. While exact integration is expected to be intractable, a number of methods have been developed that attempt to compute the posterior expectation approximately. Two main strategies are Markov chain Monte Carlo (see, e.g., the surveys in [4, 34, 60]) and variational inference (e.g., [16, 45, 67]). Markov chain Monte Carlo usually requires a large number of steps to get an accurate approximation. Further, despite remarkable mathematical progress, proving Markov chain mixing remains extremely challenging [44]. For instance, we have near-linear-time algorithms that estimate the signal $\boldsymbol{x} \in\{ \pm 1\}^{n}$ in the model (1.1) (and indeed more generally), with Bayes optimal error [50] for any $\lambda>1$. On the other hand, polynomial mixing for the posterior $p_{\boldsymbol{x} \mid \boldsymbol{Y}}$ is - to the best of our knowledge-open for any $\lambda=O(1)$.

Variational inference attempts to compute the marginals of a high-dimensional distribution by minimizing a suitable "free energy" function. This approach is usually faster than MCMC, leading to a broad range of applications, from topic modeling [15] to computer vision and inference in graphical models [42]. Of course, its accuracy relies in a crucial way on the accuracy of the free energy construction. For instance, several applications make use of the so-called "naive mean field" free energy [15]. However, it was shown in [37] that, for topic modeling (and even the simpler $\mathbb{Z}_{2}$ synchronization problem), naive mean field can return wrong information about the posterior distribution. Theorem 1.3 of this paper confirms this conclusion by showing that naive mean field does not compute the correct posterior mean for $\mathbb{Z}_{2}$ synchronization when $\lambda$ is a large enough constant.

Methods from spin glass theory [46] can overcome these limitations. Consider the Gibbs measure of the Sherrington-Kirkpatrick (SK) model of spin glasses [41], with ferromagnetic bias aligned with $\boldsymbol{x}$,

$$
\begin{aligned}
G_{\beta, \lambda}(\boldsymbol{\sigma}) & =\frac{1}{Z_{n}(\beta, \lambda)} \exp \{\beta\langle\boldsymbol{\sigma}, \boldsymbol{Y} \boldsymbol{\sigma}\rangle / 2\} \\
& =\frac{1}{Z_{n}(\beta, \lambda)} \exp \left\{\beta \lambda\langle\boldsymbol{\sigma}, \boldsymbol{x}\rangle^{2} / 2+\beta\langle\boldsymbol{\sigma}, \boldsymbol{W} \boldsymbol{\sigma}\rangle / 2\right\} .
\end{aligned}
$$

It is easy to check that the posterior distribution of $\boldsymbol{x}$ given observation $\boldsymbol{Y}$ is given by the biased SK measure with $\beta=\lambda$

$$
p(\boldsymbol{\sigma} \mid \boldsymbol{Y})=G_{\lambda, \lambda}(\boldsymbol{\sigma}) \propto \exp \{\lambda\langle\boldsymbol{\sigma}, \boldsymbol{Y} \boldsymbol{\sigma}\rangle / 2\} .
$$

\footnotetext{
${ }^{1}$ The Bayes estimator under uniform prior is minimax optimal over $x \in\{+1,-1\}^{n}$.

${ }^{2}$ This follows from Pythagora's theorem: $\mathbb{E}\left[\left\|\widehat{\boldsymbol{X}}(\boldsymbol{Y})-\boldsymbol{x} \boldsymbol{x}^{\top}\right\|_{F}^{2}\right]=\mathbb{E}\left[\left\|\widehat{\boldsymbol{X}}(\boldsymbol{Y})-\mathbb{E}\left[\boldsymbol{x} \boldsymbol{x}^{\top} \mid \boldsymbol{Y}\right]\right\|_{F}^{2}\right]+\mathbb{E}\left[\| \boldsymbol{x} \boldsymbol{x}^{\top}-\right.$ $\left.\mathbb{E}\left[\boldsymbol{x} \boldsymbol{x}^{\top} \mid \boldsymbol{Y}\right] \|_{F}^{2}\right]$.

${ }^{3}$ In physics language, the condition $\beta=\lambda$ is referred to as the "Nishimori line" [51].
} 
Following the statistical physics terminology, we shall refer to the variables $\sigma_{1}, \ldots, \sigma_{n}$ as "spins."

For the SK model, naive mean field is known to be a poor approximation of the actual free energy which explains the failures mentioned above. In 1977, Thouless, Anderson and Palmer [65] proposed a variational formula for the SK free energy, whose decision variables $\boldsymbol{m}=\left(m_{1}, \ldots, m_{n}\right) \in[-1,1]^{n}$ encode the expectation of the spins $\boldsymbol{\sigma}$. This variational formula is known as the TAP free energy, and its first-order stationarity conditions are known as the TAP equations. The relationship between the TAP equations and the Gibbs measure has been studied in the physics and mathematics literature for the last 40 years and still presents a number of outstanding challenges. A brief overview is presented in Section 2.

Explicitly, the TAP free energy for the SK model (1.4) is the function $\mathcal{F}_{\beta, \lambda}:[-1,1]^{n} \rightarrow \mathbb{R}$, defined by

$$
\mathcal{F}_{\beta, \lambda}(\boldsymbol{m})=-\frac{1}{n} \sum_{i=1}^{n} h\left(m_{i}\right)-\frac{\beta}{2 n}\left\langle\boldsymbol{Y}, \boldsymbol{m} \boldsymbol{m}^{\top}\right\rangle-\frac{\beta^{2}}{4}[1-Q(\boldsymbol{m})]^{2},
$$

where $Q(\boldsymbol{m})=\|\boldsymbol{m}\|_{2}^{2} / n$, and $h:[-1,1] \rightarrow \mathbb{R}$ is the binary entropy function

$$
h(m)=-\frac{1+m}{2} \log \left(\frac{1+m}{2}\right)-\frac{1-m}{2} \log \left(\frac{1-m}{2}\right) .
$$

The corresponding TAP equations (first order stationary condition for $\mathcal{F}_{\beta, \lambda}$ ) are given by

$$
\boldsymbol{m}=\tanh \left(\beta \boldsymbol{Y} \boldsymbol{m}-\beta^{2}[1-Q(\boldsymbol{m})] \boldsymbol{m}\right) .
$$

The first two terms in equation (1.6) correspond to the naive mean field free energy, while the term $-\beta^{2}(1-Q(\boldsymbol{m}))^{2} / 4$ is known as "Onsager's correction" and is the main innovation introduced in [65]. Indeed, heuristically, the TAP free energy can be understood using Plefka's expansion [59] which provides a series expansion of the log partition function. The first three terms of the expansion correspond to $\mathcal{F}_{\beta, \lambda}(\boldsymbol{m})$, and the others are expected to be negligible as $n \rightarrow \infty$.

Statistical physicists suggest an ambitious general conjecture on the role of the TAP free energy [46]. Namely, the Gibbs measure (1.4) is a convex combination of pure states which are well-concentrated probability measures ${ }^{4}$ with each pure state $\alpha$ corresponding to a local minimum $\boldsymbol{m}^{\alpha}$ of the TAP free energy. We refer to Section 2 for further pointers to this line of work.

Understanding the geometry of the critical points of the TAP free energy $\mathcal{F}_{\beta, \lambda}$ would help us elucidate the structure of the Gibbs measure $G_{\beta, \lambda}$. A remarkable sequence of papers within the statistical mechanics literature $[19,23,26-28,55]$ computed the expected number of critical points of the TAP free energy for the SK model $(\lambda=0)$ using nonrigorous but sophisticated tools from physics. More precisely, these authors obtain the exponential growth rate of this expectation, as a function of the free energy (the value of $\mathcal{F}_{\beta, \lambda}(\boldsymbol{m})$ ), a quantity that is known as the "complexity" of the spin model. In our first main result we prove rigorously that the formula given by [28] is indeed an upper bound for the expected number of critical points in the general setting $\lambda \geq 0$. To the best of our knowledge, similar rigorous calculations of the complexity were only obtained so far for (mixed) $p$-spin spherical models and for models on the torus. These models are simpler because of the underlying symmetry [5-7, 13].

In the context of the Bayesian model (1.5), the above picture simplifies. Adopting the jargon of statistical physics, the model is known to be replica symmetric [32] which amounts to

\footnotetext{
${ }^{4}$ A pure state $G_{\beta, \lambda}^{\alpha}$ can be assumed to be a probability measure of the form $G_{\beta, \lambda}^{\alpha}(\boldsymbol{\sigma}) \propto G_{\beta, \lambda}(\boldsymbol{\sigma}) \mathbf{1}_{\sigma \in \Omega_{\alpha}}$.
} 
say that the Gibbs measure $G_{\lambda, \lambda}(\sigma)$ is well approximated by a single pure state. As a consequence, we expect the global minimum of $\mathcal{F}_{\lambda, \lambda}$ to correspond to the posterior expectation. Our second main result proves this conjecture for all $\lambda$ larger than a big enough constant. This implies that minimizing the TAP free energy is a viable approach to optimal estimation in the present model.

While the relevance of the TAP free energy for statistical inference was pointed out several times in the past (see Section 2), this is the first rigorous result confirming this connection.

1.1. The complexity of the TAP free energy. For $U \subseteq \mathbb{R}^{4}$ and $V \subseteq(-1,1)$, define the number of critical points of $\mathcal{F}_{\beta, \lambda}$ with $\boldsymbol{m}$ in the region defined by $U$ and $V$ by

$$
\operatorname{Crit}_{n}(U, V)=\sum_{\boldsymbol{m}: \nabla \mathcal{F}_{\beta, \lambda}(\boldsymbol{m})=\mathbf{0}} \mathbf{1}\left\{(Q(\boldsymbol{m}), M(\boldsymbol{m}), A(\boldsymbol{m}), E(\boldsymbol{m})) \in U, \boldsymbol{m} \in V^{n}\right\},
$$

where $Q, M, A, E$ are the functions

$$
\begin{aligned}
Q(\boldsymbol{m}) & =\frac{1}{n}\|\boldsymbol{m}\|_{2}^{2}, \\
M(\boldsymbol{m}) & =\frac{1}{n}\langle\boldsymbol{x}, \boldsymbol{m}\rangle, \\
A(\boldsymbol{m}) & =\frac{1}{n} \sum_{i=1}^{n} m_{i} \operatorname{arctanh}\left(m_{i}\right), \\
E(\boldsymbol{m}) & =-\frac{1}{n} \sum_{i=1}^{n}\left[h\left(m_{i}\right)+\frac{1}{2} m_{i} \operatorname{arctanh}\left(m_{i}\right)\right]-\frac{\beta^{2}}{4}\left(1-Q(\boldsymbol{m})^{2}\right) .
\end{aligned}
$$

Note that at any point $\boldsymbol{m}$ where $\nabla \mathcal{F}_{\beta, \lambda}(\boldsymbol{m})=\mathbf{0}$, we have from equation (1.7) that $\mathcal{F}_{\beta, \lambda}(\boldsymbol{m})=$ $E(\boldsymbol{m})$.

Define the function $S_{\star}:(0,1] \times[-1,1] \times \mathbb{R}^{2} \rightarrow \mathbb{R}$ by

$$
S_{\star}(q, \varphi, a, e)=\inf _{(\mu, \nu, \tau, \gamma) \in \mathbb{R}^{4}} S(q, \varphi, a, e ; \mu, \nu, \tau, \gamma),
$$

where

$$
S(q, \varphi, a, e ; \mu, v, \tau, \gamma)=\frac{1}{4 \beta^{2}}\left[\frac{a}{q}-\frac{\beta \lambda \varphi^{2}}{q}-\beta^{2}(1-q)\right]^{2}
$$

$$
-q \mu-\varphi v-a \tau-[u(q, a)-e] \gamma+\log I(q, \varphi, \mu, v, \tau, \gamma),
$$

and

$$
u(q, a)=-\frac{\beta^{2}}{4}\left(1-q^{2}\right)+\frac{a}{2}
$$

$$
\begin{aligned}
& I(q, \varphi, \mu, \nu, \tau, \gamma) \\
& =\int_{-\infty}^{\infty} \frac{1}{\left(2 \pi \beta^{2} q\right)^{1 / 2}} \exp \left\{-\frac{(x-\beta \lambda \varphi)^{2}}{2 \beta^{2} q}\right. \\
& \left.\quad+\mu \tanh ^{2}(x)+v \tanh (x)+\tau x \tanh (x)+\gamma \log [2 \cosh (x)]\right\} \mathrm{d} x .
\end{aligned}
$$

TheOREM 1.1. Fix any $\beta>0, \lambda \geq 0, \eta, b \in(0,1)$ and closed set $U \subseteq[\eta, 1] \times \mathbb{R}^{3}$. Let $V_{n}=\left[-1+e^{-n^{b}}, 1-e^{-n^{b}}\right]$. Then,

$$
\limsup _{n \rightarrow \infty} n^{-1} \log \mathbb{E}\left[\operatorname{Crit}_{n}\left(U, V_{n}\right)\right] \leq \sup _{(q, \varphi, a, e) \in U} S_{\star}(q, \varphi, a, e) .
$$


The main proof strategy of Theorem 1.1 is to calculate $\mathbb{E}\left[\mathrm{Crit}_{n}\left(U, V_{n}\right)\right]$ using the KacRice formula [1], Theorem 11.2.1. This is the same strategy as pioneered by Auffinger, Ben Arous and Černỳ in [7] (building on early insights by Fyodorov [36]) in the context of the spherical $p$-spin model. However, several new challenges arise. First of all, we cannot adopt [1], Theorem 11.2.1, directly because of a degeneracy of the conditional Hessian. We present a proof for the Kac-Rice upper bound with degeneracy in Appendix A.

We then evaluate the Kac-Rice formula to leading exponential order. As usual, the most difficult step requires to evaluate the expected determinant of the Hessian. In the spherical model this is distributed as the determinant of a Wigner matrix, shifted by a term proportional to the identity, and an exact formula was given in [7]. A slightly more complicated calculation arises for the $p$-spin spherical model with biased coupling (see [13]), where the Hessian is a low-rank deformation of a Wigner matrix shifted by a term proportional to the identity. In the present case the Hessian is distributed as a low-rank deformation of a Wigner matrix plus a diagonal matrix which depends on the point $\boldsymbol{m} \in[-1,+1]^{n}$. Unlike in earlier work, the bulk of the spectral distribution of the Hessian depends now on the point $\boldsymbol{m}$ (instead of $\|\boldsymbol{m}\|_{2}$ ). We give an upper bound of the expected determinant of the Hessian using free probability theory in Section 4. Finally, we use this expression to upper bound the exponential growth rate of $\mathbb{E}\left[\mathrm{Crit}_{n}\left(U, V_{n}\right)\right]$ using Sanov's theorem and Varadhan's lemma in Appendix B. We refer to Section 3 for details of the proof.

REMARK 1.1. We impose the technical conditions that $U \subseteq[\eta, 1] \times \mathbb{R}^{3}$ and $V_{n}=[-1+$ $e^{-n^{b}}, 1-e^{-n^{b}}$ ] because of the singular behaviors of the TAP free energy at zero and at the boundary which are subtle to deal with using the Kac-Rice formula. Instead, the behaviors of $\mathcal{F}_{\beta, \lambda}$ near zero and near the boundary can be easily analyzed by direct arguments.

Indeed, $\boldsymbol{m}=\mathbf{0}$ is always a critical point of $\mathcal{F}_{\beta, \lambda}$. In one interesting case $\beta>1$ and $\lambda=0$, it can be easily shown that $\mathcal{F}_{\beta, \lambda}$ is strongly convex over $D_{\eta} \equiv\left\{\boldsymbol{m} \in[-1,1]^{n}: Q(\boldsymbol{m}) \leq \eta\right\}$ with high probability, so that there are no other critical points of $\mathcal{F}_{\beta, \lambda}$ inside $D_{\eta}$. In another interesting case, namely, when $\beta=\lambda$ are large enough, the critical points inside $D_{\eta}$ have high function value with high probability (see Lemma C.1), so that they are not relevant.

Near the boundary, $\nabla \mathcal{F}_{\beta, \lambda}$ diverges due to the entropy term. It is easy to show that there are no critical points of $\mathcal{F}_{\beta, \lambda}$ within the region $\left\{\boldsymbol{m}:\left|\|\boldsymbol{m}\|_{\infty}-1\right| \leq \exp \left(-n^{2 / 3}\right)\right\}$ with high probability for sufficiently large $n$. (See Lemma 5.4 for the proof of the case $\beta=\lambda$.)

1.2. Consequences for $S K$ model with zero-mean couplings $(\lambda=0)$. It is interesting to specialize Theorem 1.1 to $\lambda=0$ (the SK model with zero-mean couplings) and, analytically, maximize over $\varphi$ (the value of the magnetization) which is equivalent to setting $v=\varphi=0$. Finally, we replace the variable $a$ by $\Delta$ via $a=\beta^{2} q(1-q)+2 q \Delta$. This results in the reduced expression

$$
\begin{aligned}
S_{0, \star, \beta}(q, \Delta, e)= & \inf _{(\mu, \tau, \gamma) \in \mathbb{R}^{3}} S_{0}(q, \Delta, e ; \mu, \tau, \gamma), \\
S_{0}(q, \Delta, e ; \mu, \tau, \gamma)= & \frac{\Delta^{2}}{\beta^{2}}-q \mu+e \gamma-\left(\tau+\frac{\gamma}{2}\right)\left(\beta^{2} q(1-q)+2 q \Delta\right) \\
& +\frac{\beta^{2}}{4}\left(1-q^{2}\right) \gamma+\log I_{0}(q, \mu, \tau, \gamma), \\
I_{0}(q, \mu, \tau, \gamma)= & \int_{-\infty}^{\infty} \frac{1}{\left(2 \pi \beta^{2} q\right)^{1 / 2}} \exp \left\{-\frac{x^{2}}{2 \beta^{2} q}\right. \\
& \left.+\mu \tanh ^{2}(x)+\tau x \tanh (x)+\gamma \log [2 \cosh (x)]\right\} \mathrm{d} x .
\end{aligned}
$$


This coincides with the expression in the statistical physics literature; cf. [19, 23, 2628, 55]. We refer in particular to [26] which compares different theoretical physics approaches. Equations (29)-(31) of [26] can be recovered from the above expression by setting $\mu=\lambda_{\mathrm{CLPR}}-\left(\Delta^{2} /\left(2 \beta^{2} q\right)\right), \gamma=-u_{\mathrm{CLPR}}, \tau=\left(u_{\mathrm{CLPR}} / 2\right)+\left(\Delta /\left(\beta^{2} q\right)\right)$. Minimization over $(\mu, \tau, \gamma)$ is then replaced by minimization over $\left(\lambda_{\mathrm{CLPR}}, u_{\mathrm{CLPR}}\right)$.

Notice that the expression of [26] involves one extra parameter (denoted by $B$ ). However, the authors set it to 0 on the basis of physical considerations motivated by "Plefka's criterion" [59].

Denote by $H_{\mathrm{SK}}(\boldsymbol{\sigma})=-\langle\boldsymbol{\sigma}, \boldsymbol{W} \boldsymbol{\sigma}\rangle / 2$ (where $\sigma \in\{+1,-1\}^{n}$ ) the Hamiltonian of the SK model. Substituting $\boldsymbol{m}=\boldsymbol{\sigma}$ in equation (1.6), we obtain the following lower bound on the ground state energy:

$$
\frac{1}{n} \min _{\sigma \in\{-1,+1\}^{n}} H_{\mathrm{SK}}(\boldsymbol{\sigma}) \geq \frac{1}{\beta} \min _{\boldsymbol{m} \in[-1,+1]^{n}} \mathcal{F}_{\beta, 0}(\boldsymbol{m}) .
$$

Note that $(1 / n) \min _{\sigma \in\{-1,+1\}^{n}} H_{\mathrm{SK}}(\sigma)$ concentrates exponentially around its expectation by Gaussian concentration [18]. Using Markov's inequality and the fact that local minima of $\mathcal{F}_{\beta, 0}(\boldsymbol{m})$ occur in the interior of $[-1,+1]^{n}$ (see Lemma 5.4), we get the following lower bound on the expected ground state energy:

$$
\begin{aligned}
& \lim \inf _{n \rightarrow \infty} \frac{1}{n} \mathbb{E} \min _{\sigma \in\{-1,+1\}^{n}} H_{\mathrm{SK}}(\sigma) \\
& \geq F_{1 \mathrm{RSB}}(\beta) \equiv \frac{1}{\beta} \inf \left\{e: \sup _{q, \Delta} S_{0, \star, \beta}(q, \Delta, e) \geq 0\right\} .
\end{aligned}
$$

(Here, $S_{0, \star, \beta}(q, \Delta, e)$ is given by equation (1.14).) In [23], Cavagna, Giardina, Parisi and Mézard identify $F_{1 \mathrm{RSB}}(\beta)$ with the "one step replica symmetry breaking" (1RSB) formula for the free energy of the SK model. Hence, our result provides an alternative route to prove the celebrated 1RSB lower bound-first established by Guerra in [38] — in the zero temperature $(\beta=\infty)$ case.

1.3. Bayes estimation in $\mathbb{Z}_{2}$ synchronization. We now return to the $\mathbb{Z}_{2}$ synchronization model of equation (1.1). As mentioned above, the posterior distribution of $\boldsymbol{x}$ given observation $\boldsymbol{Y}$ is given (under the uniform prior) by the biased SK Gibbs measure (1.4) with $\beta=\lambda$; cf. equation (1.5). Accordingly, we fix $\beta=\lambda$ throughout this section.

Given the relation between the TAP free energy and the Bayes posterior, it is natural to consider the estimator

$$
\widehat{\boldsymbol{x}}^{\mathrm{TAP}}(\boldsymbol{Y}) \equiv \underset{\boldsymbol{m} \in[-1,+1]^{n}}{\arg \min } \mathcal{F}_{\lambda, \lambda}(\boldsymbol{m}) .
$$

Our next theorem provides a characterization of the landscape of the TAP free energy $\mathcal{F}_{\lambda, \lambda}$ for $\lambda$ a sufficiently large constant, implying in particular that $\widehat{\boldsymbol{x}}^{\mathrm{TAP}}(\boldsymbol{Y})$ is close to the Bayes optimal estimator. More precisely, we show that any critical point $\boldsymbol{m}$ for which $\mathcal{F}_{\lambda, \lambda}(\boldsymbol{m})$ is below a certain constant energy value is such that $\boldsymbol{m} \boldsymbol{m}^{\top} \approx \widehat{\boldsymbol{X}}_{\text {Bayes. }}$. This implies that any such $\mathbf{m} \boldsymbol{m}^{\top}$ also asymptotically achieves the Bayes risk $\operatorname{MMSE}(\lambda)$.

\section{THEOREM 1.2. Denote}

$$
\mathcal{C}_{\lambda, n}=\left\{\boldsymbol{m} \in[-1,1]^{n}: \nabla \mathcal{F}_{\lambda, \lambda}(\boldsymbol{m})=\mathbf{0}, \mathcal{F}_{\lambda, \lambda}(\boldsymbol{m}) \leq-\lambda^{2} / 3\right\} .
$$

There exists a constant $\lambda_{0}>0$ such that, for any constant $\lambda>\lambda_{0}$, we have $\mathcal{C}_{\lambda, n} \neq \varnothing$ with high probability, and

$$
\lim _{n \rightarrow \infty} \mathbb{E}\left[\left(\sup _{\boldsymbol{m} \in \mathcal{C}_{\lambda, n}} \frac{1}{n^{2}}\left\|\boldsymbol{m} \boldsymbol{m}^{\top}-\widehat{\boldsymbol{X}}_{\text {Bayes }}\right\|_{F}^{2}\right) \mathbf{1}\left\{\mathcal{C}_{\lambda, n} \neq \varnothing\right\}\right]=0 .
$$


We refer to Section 5 for the proof of this theorem.

This theorem suggests that a good minimizer of $\mathcal{F}_{\lambda, \lambda}$ can be computed by applying a gradient based optimization algorithm, provided the initialization has a value of $\mathcal{F}_{\lambda, \lambda}$ below a constant. It is easy to construct an initialization satisfying the condition required here. For instance, if $\boldsymbol{v}_{1}(\boldsymbol{Y})$ is the principal eigenvector of $\boldsymbol{Y}$ with unit norm, we can set $\boldsymbol{m}^{0}=\operatorname{sign}\left(\boldsymbol{v}_{1}(\boldsymbol{Y})\right)$. A simple calculation yields $\mathcal{F}_{\lambda, \lambda}\left(\boldsymbol{m}^{0}\right)=-(\lambda / 2 n)\left\langle\boldsymbol{Y}, \boldsymbol{m}^{0}\left(\boldsymbol{m}^{0}\right)^{\top}\right\rangle \leq$ $-\left(\lambda^{2} / 2 n^{2}\right)\left\langle\boldsymbol{m}^{0}, \boldsymbol{x}\right\rangle^{2}+\lambda$ (where the upper bound holds with high probability, from $\|\boldsymbol{W}\|_{\text {op }} \leq 2$ [35]). Using standard results on the eigenvectors of deformed GOE matrices [9], we get $\left|\left\langle\boldsymbol{m}^{0}, \boldsymbol{x}\right\rangle\right| / n \geq 1-\varepsilon$ for all $\lambda>\lambda_{0}(\varepsilon)$ whence $\mathcal{F}_{\lambda, \lambda}\left(\boldsymbol{m}^{0}\right)<-\lambda^{2} / 3$ with high probability for all $\lambda$ large enough.

REMARK 1.2. Since the TAP free energy is nonconvex, the above does not prove that it can be optimized efficiently; further information about its Hessian would be required to establish this. However, the papers $[32,50]$ develop an approximate message passing (AMP) algorithm that attempts to construct the solution of the TAP equation (1.7). The algorithm is iterative and proceeds as follows, with $\boldsymbol{m}^{-1}=\mathbf{0}$ :

$$
\begin{aligned}
\boldsymbol{m}^{0} & =\tanh \left(c_{0}(\lambda) \sqrt{n} \boldsymbol{v}_{1}(\boldsymbol{Y})\right), \\
\boldsymbol{m}^{k+1} & =\tanh \left(\lambda \boldsymbol{Y} \boldsymbol{m}^{k}-\lambda^{2}\left[1-Q\left(\boldsymbol{m}^{k}\right)\right] \boldsymbol{m}^{k-1}\right) .
\end{aligned}
$$

Here, $\boldsymbol{v}_{1}(\boldsymbol{Y})$ is the principal eigenvector of $\boldsymbol{Y}$ with unit norm, and $c_{0}(\lambda)$ is a suitable normalization (see [50]). Building on earlier work by Bolthausen [17] as well as on [32], it is shown in [50] that the iterates $\boldsymbol{m}^{k}$ asymptotically achieve the Bayes risk, for any $\lambda>1$,

$$
\lim _{k \rightarrow \infty} \lim _{n \rightarrow \infty} \frac{1}{n^{2}}\left\|\boldsymbol{m}^{k}\left(\boldsymbol{m}^{k}\right)^{\top}-\boldsymbol{x} \boldsymbol{x}^{\top}\right\|_{F}^{2}=\lim _{n \rightarrow \infty} \operatorname{MMSE}_{n}(\lambda) .
$$

Together with Theorem 1.2, this result implies that the algorithm is constructing an approximation of $\widehat{\boldsymbol{x}}^{\mathrm{TAP}}(\boldsymbol{Y})$,

$$
\lim _{k \rightarrow \infty} \lim _{n \rightarrow \infty} \mathbb{E}\left[\frac{1}{n^{2}}\left\|\boldsymbol{m}^{k}\left(\boldsymbol{m}^{k}\right)^{\top}-\widehat{\boldsymbol{x}}^{\mathrm{TAP}}\left(\widehat{\boldsymbol{x}}^{\mathrm{TAP}}\right)^{\top}\right\|_{F}^{2}\right]=0 .
$$

This leaves open two interesting questions: $(i)$ Can we construct an algorithm that converges to $\widehat{\boldsymbol{x}}^{\mathrm{TAP}}(\boldsymbol{Y})$, for fixed $n$ (i.e.. can we invert the order of limits in the last equation)? (ii) Do generic optimization algorithms (e.g., gradient descent) converge to $\widehat{\boldsymbol{x}}^{\mathrm{TAP}}(\boldsymbol{Y})$ when applied to $\mathcal{F}_{\lambda, \lambda}$ ?

REMARK 1.3. While-as discussed in the last remark-the AMP algorithm of [50] achieves the Bayes risk, the optimization-based formulation of equation (1.19) has important practical advantages. In particular, we do not know how robust is AMP with respect to deviations from the model (1.1). On the other hand, we expect $\widehat{\boldsymbol{x}}^{\mathrm{TAP}}(\boldsymbol{Y})$ to be robust and meaningful even if the model for the data $\boldsymbol{Y}$ is incorrect.

The TAP free energy (1.6) may be contrasted with the "mean field free energy"

$$
\mathcal{F}_{\mathrm{MF}}(\boldsymbol{m})=-\frac{1}{n} \sum_{i=1}^{n} h\left(m_{i}\right)-\frac{\lambda}{2 n}\langle\boldsymbol{m}, \boldsymbol{Y} \boldsymbol{m}\rangle,
$$

whose stationary points satisfy the mean field equations

$$
\boldsymbol{m}=\tanh (\lambda \boldsymbol{Y} \boldsymbol{m}) .
$$

Notice that the mean field equations omit the Onsager correction term $-\lambda^{2}[1-Q(\boldsymbol{m})] \boldsymbol{m}$. To clarify the importance of this correction term, we establish the following negative result for critical points $\boldsymbol{m}$ of $\mathcal{F}_{\mathrm{MF}}$. 
THEOREM 1.3. Denote $\mathcal{S}_{\lambda, n}=\left\{\boldsymbol{m} \in(-1,1)^{n}: \nabla \mathcal{F}_{\mathrm{MF}}(\boldsymbol{m})=\mathbf{0}\right\}$. There exists a constant $\lambda_{0}>0$ and a constant $\varepsilon(\lambda)>0$ for every $\lambda>\lambda_{0}$, such that

$$
\lim _{n \rightarrow \infty} \mathbb{P}\left(\inf _{\boldsymbol{m} \in \mathcal{S}_{\lambda, n}} \frac{1}{n^{2}}\left\|\boldsymbol{m m}^{\top}-\widehat{\boldsymbol{X}}_{\text {Bayes }}\right\|_{F}^{2} \geq \varepsilon(\lambda)\right)=1 .
$$

The proof of this theorem is presented in Appendix D.

1.4. Notation. We use boldface for vectors and matrices, for example, $\boldsymbol{X}$ for a matrix and $\boldsymbol{x}$ for a vector. For a univariate function $f: \mathbb{R} \rightarrow \mathbb{R}$ and a vector $\boldsymbol{x}=\left(x_{1}, x_{2}, \ldots, x_{n}\right)^{\top} \in \mathbb{R}^{n}$, we define the function $f: \mathbb{R}^{n} \rightarrow \mathbb{R}^{n}$ by $f(\boldsymbol{x})=\left(f\left(x_{1}\right), f\left(x_{2}\right), \ldots, f\left(x_{n}\right)\right)^{\top}$. For example, we write $\boldsymbol{x}^{2}=\left(x_{1}^{2}, x_{2}^{2}, \ldots, x_{n}^{2}\right)^{\mathrm{T}}$. We denote $\mathrm{B}^{n}(\boldsymbol{x}, r)$ to be the Euclidean ball in $\mathbb{R}^{n}$ centered at $\boldsymbol{x} \in \mathbb{R}^{n}$ with radius $r$.

2. Related literature. The Sherrington-Kirkpatrick (SK) model [41] is a mean field model of spin glasses. The asymptotics of its $\log$-partition function $\lim _{n \rightarrow \infty} n^{-1} \log Z_{n}(\beta, \lambda)$ (cf. equation (1.4)) was first computed using the replica method [52-54] and is expressed using the so-called Parisi's formula. Thouless, Anderson and Palmer [65] proposed a variational principle in terms of the TAP free energy of equation (1.6).

The relationship between the Gibbs measure (1.4) (and the partition function) and the TAP free energy has been studied in the physics literature [20, 23, 30]. The local minimizers of the TAP free energy are interpreted as the local magnetizations of pure states. As a partial confirmation of this prediction, Talagrand [64] and Chatterjee [24] proved that the TAP equation holds for local magnetizations at high temperature (up to a small error term). Bolthausen [17] derived an iterative algorithm for solving the TAP equations at high temperature. This is an instance of a broader class of iterative algorithms that are now known as approximate message passing (AMP); see, for example, [12]. At the low temperature regime, Auffinger and Jagannath [8] proved that the TAP equations are satisfied, approximately, by local magnetizations of pure states. Recently, Chen and Panchenko [25] proved that the minimum of the TAP free energy (under a suitable constraint on $\boldsymbol{m}$ ) coincides indeed with the normalized $\log$ partition function $n^{-1} \log Z_{n}(\beta, \lambda)$ up to an error vanishing as $n \rightarrow \infty$.

The exponential growth rate of the expected number of critical points of the TAP free energy is also known as the "annealed complexity." It was first computed by Bray and Moore in [19] using the replica method, while an alternative "supersymmetric" approach was developed in [23]. The two methods give equivalent formal expressions which correspond to the function $S_{0}$ of equation (1.15). These two papers, however, report different results for the overall complexity as a function of the free energy which follow from two different ways to set the Lagrange parameters in $S_{0}$. This discrepancy was further investigated in [26] and [28] which suggest that the specific choice should depend on the value of the free energy. This is consistent with the prescription of our Theorem 1.1.

So far, the annealed complexity function has been computed rigorously for the spherical $p$-spin model [7], for the spherical mixed $p$-spin model [6] and for certain Gaussian energy functions over the torus [5]. For the spherical $p$-spin model, Subag [63] used the second moment method to show that the typical number of critical points coincides with its expected value for energies in a certain interval. A version of the spherical $p$-spin model with nonvanishing mean was studied in [13]. This model has a statistical interpretation analogous to the one of model (1.1). As mentioned above, the main technical challenge in extending these results to the SK model is to compute the conditional expectation of the absolute value of the determinant of the Hessian. (Let us mention that — apart from using nonrigorous methodsall of the physics derivations drop this absolute value, without justification.) Our calculation of this quantity relies on tools from free probability theory $[14,22,66]$. 
The $\mathbb{Z}_{2}$ synchronization problem has proven to be a useful testing ground for comparing different approaches. The Bayes risk was computed in [32]. In particular, a nontrivial correlation with the signal can only be achieved for $\lambda>1$. The ideal threshold $\lambda=1$ is achieved both by spectral methods [9] and semidefinite programming [40, 49]. However, neither of these approaches achieves the Bayes optimal error.

Over the last few years a substantial amount of work has been devoted to models that generalize $\mathbb{Z}_{2}$ synchronization (1.1) in significant ways. We refer to [2, 11, 33, 43, 47, 50, 58] for a few pointers to this literature. Among other settings, these authors consider the model $\boldsymbol{Y}=(\lambda / n) \boldsymbol{X} \boldsymbol{X}^{\top}+\boldsymbol{W}$, where $\boldsymbol{X} \in \mathbb{R}^{n \times r}$ is a signal with fixed rank $r$ and i.i.d. rows $\left(\boldsymbol{X}_{\cdot, i}\right)_{i \leq n}$ with known distribution $p_{X}$ over $\mathbb{R}^{r}$. Both Bayes optimal error and the performance of optimal message passing algorithms have been characterized in this context. The goal of the present paper is quite different: we want to understand the optimality of an optimizationbased method which uses the TAP free energy as the cost function. As explained in Remark 1.3, such an approach has significant practical advantages.

Variational methods based on free-energy approximations have been the object of recent interest within the statistics literature. Zhang and Zhou [68] study mean field variational inference applied to the stochastic block model. They prove optimal error rates in a regime that is equivalent to the $\lambda \rightarrow \infty$ regime for $\mathbb{Z}_{2}$ synchronization. In the opposite direction, [37] shows that, for $\lambda \in(1 / 2,1)$, the minimizer of the mean field free energy does not approximate the Bayes posterior mean. Replacing the mean field with the TAP free energy solves this problem.

3. Proof of Theorem 1.1. In this section we prove Theorem 1.1. By symmetry with respect to the sign change for any coordinate

$$
x_{i} \mapsto-x_{i}, \quad m_{i} \mapsto-m_{i},
$$

we may assume without loss of generality throughout the proof of Theorem 1.1 that $\boldsymbol{x}=\mathbf{1}$. Let $f_{n}(\boldsymbol{m})=n \mathcal{F}_{\beta, \lambda}(\boldsymbol{m})$ be the scaled TAP free energy. Then, $f_{n}$ and its gradient and Hessian are given by

$$
f_{n}(\boldsymbol{m})=-\sum_{i=1}^{n} h\left(m_{i}\right)-\frac{\beta \lambda}{2 n}\langle\mathbf{1}, \boldsymbol{m}\rangle^{2}-\frac{\beta}{2}\left\langle\boldsymbol{W}, \boldsymbol{m} \boldsymbol{m}^{\top}\right\rangle-\frac{n \beta^{2}}{4}[1-Q(\boldsymbol{m})]^{2},
$$

$$
\begin{aligned}
& \boldsymbol{g}_{n}(\boldsymbol{m})=\operatorname{arctanh}(\boldsymbol{m})-\frac{\beta \lambda}{n}\langle\mathbf{1}, \boldsymbol{m}\rangle \mathbf{1}-\beta \boldsymbol{W} \boldsymbol{m}+\beta^{2}[1-Q(\boldsymbol{m})] \boldsymbol{m} \\
& \boldsymbol{H}_{n}(\boldsymbol{m})=\operatorname{diag}\left(1 /\left(1-\boldsymbol{m}^{2}\right)\right)-\frac{\beta \lambda}{n} \mathbf{1 1}^{\top}-\beta \boldsymbol{W}+\beta^{2}[1-Q(\boldsymbol{m})] \mathbf{I}_{n}-\frac{2 \beta^{2}}{n} \boldsymbol{m}^{\top}
\end{aligned}
$$

3.1. Kac-Rice formula. Using a version of the Kac-Rice formula, we prove the following statement in Appendix A.

Proposition 3.1. Fix any $\beta>0$ and $\lambda \geq 0$. Denote by $p_{\boldsymbol{m}}(\mathbf{0})$ the Lebesgue density of $\boldsymbol{g}_{n}(\boldsymbol{m})$ at $\mathbf{0}$. Then, for any $\delta \equiv \delta_{n}>0$ and any $U \subseteq[\delta, 1] \times \mathbb{R}^{3}$ and $V \subseteq[-1+\delta, 1-\delta]$, we have

$$
\begin{aligned}
\mathbb{E}\left[\operatorname{Crit}_{n}(U, V)\right] \leq & \int_{V^{n}} \mathbf{1}_{\{(Q(\boldsymbol{m}), M(\boldsymbol{m}), A(\boldsymbol{m}), E(\boldsymbol{m})) \in U\}} \\
& \times \mathbb{E}\left[\left|\operatorname{det}\left(\boldsymbol{H}_{n}(\boldsymbol{m})\right)\right| \mid \boldsymbol{g}_{n}(\boldsymbol{m})=\mathbf{0}\right] p_{\boldsymbol{m}}(\mathbf{0}) \mathrm{d} \boldsymbol{m} .
\end{aligned}
$$


3.2. Determinant of Hessian and density of gradient. We next analyze the expectation on the right side of (3.4) using techniques from random matrix theory. The proof of the following result is deferred to Section 4.

Proposition 3.2. Fix $\beta>0, \lambda \geq 0$ and $\eta, b \in(0,1)$. Then, there are constants $n_{0} \equiv$ $n_{0}(\beta, \lambda, \eta, b)>0$ and $C_{0} \equiv C_{0}(\beta, \lambda, \eta, b)$, such that the following holds for all $n \geq n_{0}$. Let $\boldsymbol{m}$ satisfy $\|\boldsymbol{m}\|_{\infty} \leq 1-e^{-n^{b}}$ and $Q(\boldsymbol{m}) \in[\eta, 1]$. Define

$$
L(\boldsymbol{m})=\frac{\beta^{2}[1-Q(\boldsymbol{m})]^{2}}{2}+\frac{1}{n} \sum_{i=1}^{n} \log \left(\frac{1}{1-m_{i}^{2}}\right) .
$$

Then,

$$
\mathbb{E}\left[\left|\operatorname{det}\left(\boldsymbol{H}_{n}(\boldsymbol{m})\right)\right| \mid \boldsymbol{g}_{n}(\boldsymbol{m})=\mathbf{0}\right] \leq \exp \left(n \cdot L(\boldsymbol{m})+C_{0} \cdot n^{\max (0.9, b)}\right) .
$$

Define

$$
\begin{aligned}
\boldsymbol{u}(\boldsymbol{m}) & =\left[u_{1}(\boldsymbol{m}), \ldots, u_{n}(\boldsymbol{m})\right]^{\top} \\
& =\operatorname{arctanh}(\boldsymbol{m})-\beta \lambda / n \cdot\langle\mathbf{1}, \boldsymbol{m}\rangle \cdot \mathbf{1}+\beta^{2}[1-Q(\boldsymbol{m})] \boldsymbol{m} .
\end{aligned}
$$

The quantity $p_{\boldsymbol{m}}(\mathbf{0})$ in (3.4) may also be evaluated as follows.

LEMMA 3.3.

$$
\begin{aligned}
p_{\boldsymbol{m}}(\mathbf{0})= & \int_{\mathbb{R}} \frac{1}{\left\{2 \pi \beta^{2} Q(\boldsymbol{m})\right\}^{n / 2}} \exp \left\{-\sum_{i=1}^{n} \frac{\left(u_{i}(\boldsymbol{m})-y m_{i}\right)^{2}}{2 \beta^{2} Q(\boldsymbol{m})}\right\} \\
& \cdot \sqrt{\frac{n}{2 \pi \beta^{2}}} \exp \left\{-\frac{n y^{2}}{2 \beta^{2}}\right\} \mathrm{d} y .
\end{aligned}
$$

PROOF. First, we observe that

$$
\mathbb{E}\left[(\boldsymbol{W} \boldsymbol{m})_{i}(\boldsymbol{W} \boldsymbol{m})_{j}\right]=Q(\boldsymbol{m}) \cdot \mathbf{1}\{i=j\}+m_{i} m_{j} / n .
$$

Therefore, we have

$$
\boldsymbol{W} \boldsymbol{m} \stackrel{d}{=} Q(\boldsymbol{m})^{1 / 2} \boldsymbol{v}+Y \cdot \boldsymbol{m},
$$

where $\boldsymbol{v} \sim \mathcal{N}\left(0, \mathbf{I}_{n}\right)$ and $Y \sim \mathcal{N}(0,1 / n)$ are independent. As a consequence, by (3.2),

$$
\boldsymbol{g}_{n}(\boldsymbol{m}) \stackrel{d}{=} \boldsymbol{u}(\boldsymbol{m})-\beta\left(Q(\boldsymbol{m})^{1 / 2} \boldsymbol{v}+Y \cdot \boldsymbol{m}\right),
$$

and the form of $p_{\boldsymbol{m}}(\mathbf{0})$ follows by first writing the density of $\boldsymbol{g}_{n}(\boldsymbol{m})$ conditional on $\beta Y$ and then integrating over the law of $\beta Y$.

Combining Proposition 3.1, Proposition 3.2 and Lemma 3.3 above, we have the following immediate corollary.

Corollary 3.4. Fix $\beta>0, \lambda \geq 0$ and $\eta, b \in(0,1)$. There exist $n_{0} \equiv n_{0}(\beta, \lambda, \eta, b)$ and $C_{0} \equiv C_{0}(\beta, \lambda, \eta, b)$ such that, for all $n \geq n_{0}$, any $U \subseteq[\eta, 1] \times \mathbb{R}^{3}$ and $V_{n}=\left[-1+e^{-n^{b}}, 1-\right.$ $\left.e^{-n^{b}}\right]$, we have

$$
\mathbb{E}\left[\operatorname{Crit}_{n}\left(U, V_{n}\right)\right] \leq T\left(U, V_{n}\right),
$$


where

$$
\begin{aligned}
T\left(U, V_{n}\right)= & \sqrt{\frac{n}{2 \pi \beta^{2}}} \int_{(-1,1)^{n} \times \mathbb{R}} \exp \left(n J(\boldsymbol{m}, y)-\frac{n y^{2}}{2 \beta^{2}}+C_{0} \cdot n^{\max (0.9, b)}\right) \\
& \cdot \mathbf{1}_{(Q(\boldsymbol{m}), M(\boldsymbol{m}), A(\boldsymbol{m}), E(\boldsymbol{m})) \in U} \mathrm{~d} \boldsymbol{m} \mathrm{d} y, \\
J(\boldsymbol{m}, y)= & \frac{\beta^{2}(1-Q(\boldsymbol{m}))^{2}}{2}-\frac{1}{2} \log \left(2 \pi \beta^{2} Q(\boldsymbol{m})\right) \\
& +\frac{1}{n} \sum_{i=1}^{n} \log g\left(m_{i} ; M(\boldsymbol{m}), Q(\boldsymbol{m}), y\right), \\
g(x ; \varphi, q, y)= & \frac{1}{1-x^{2}} \cdot \exp \left(-\frac{\left(\operatorname{arctanh}(x)-\beta \lambda \varphi+\beta^{2}(1-q) x-y x\right)^{2}}{2 \beta^{2} q}\right) .
\end{aligned}
$$

3.3. Variational upper bound. We next analyze the integral $T\left(U, V_{n}\right)$ in Corollary 3.4 using large deviations techniques. Let $\mathcal{P}$ be the space of Borel probability measures on $(-1,1)$. For $\rho \in \mathcal{P}$, define

$$
\begin{aligned}
& A(\rho)=\int_{-1}^{1} x \operatorname{arctanh}(x) \rho(\mathrm{d} x), \quad M(\rho)=\int_{-1}^{1} x \rho(\mathrm{d} x), \quad Q(\rho)=\int_{-1}^{1} x^{2} \rho(\mathrm{d} x), \\
& E(\rho)=-\int_{-1}^{1}\left[h(x)+\frac{1}{2} x \operatorname{arctanh}(x)\right] \rho(\mathrm{d} x)-\frac{\beta^{2}}{4}\left(1-Q(\rho)^{2}\right) .
\end{aligned}
$$

Here, $A(\rho)$ may equal $\infty$, and $E(\rho)$ may equal $-\infty$. For $\rho \in \mathcal{P}$ such that $Q(\rho)>0$ and for $y \in \mathbb{R}$, define the extended real-valued functional

$$
J(\rho, y)=\frac{\beta^{2}(1-Q(\rho))^{2}}{2}-\frac{1}{2} \log \left(2 \pi \beta^{2} Q(\rho)\right)+\int_{-1}^{1} \log g(x ; M(\rho), Q(\rho), y) \rho(\mathrm{d} x) .
$$

Note that $\log \left[1 /\left(1-x^{2}\right)\right]$ grows at a slower rate than $\operatorname{arctanh}(x)^{2}$ as $x \rightarrow \pm 1$, so $J(\rho, y)<$ $\infty$ always, and $J(\rho, y)>-\infty$ when $\int_{-1}^{1} \operatorname{arctanh}(x)^{2} \rho(\mathrm{d} x)<\infty$. These functionals extend the previous definitions upon identifying $\boldsymbol{m} \in(-1,1)^{n}$ with the empirical distribution of its coordinates. Further recall the definition of relative entropy between two Borel probability measures $\mu$ and $\pi$ on $\mathbb{R}$,

$$
H(\mu \mid \pi):= \begin{cases}\int \log \left(\frac{\mathrm{d} \mu}{\mathrm{d} \pi}(x)\right) \mu(\mathrm{d} x) & \text { if } \mu \ll \pi, \\ +\infty & \text { otherwise. }\end{cases}
$$

We establish the following upper bound in Appendix B.

Proposition 3.5. Fix $\beta>0, \lambda \geq 0, \eta, b \in(0,1)$ and any closed set $U \subseteq[\eta, 1] \times \mathbb{R}^{3}$. Let $V_{n}$ and $T\left(U, V_{n}\right)$ be as in Corollary 3.4 ; let $\pi_{0}$ be the uniform distribution on $(-1,1)$. Then,

$$
\begin{aligned}
& \limsup _{n} \frac{1}{n} \log T\left(U, V_{n}\right) \\
& \quad \leq \sup _{\rho \in \mathcal{P}:(Q(\rho), M(\rho), A(\rho), E(\rho)) \in U} \sup _{y \in \mathbb{R}}\left\{J(\rho, y)-H\left(\rho \mid \pi_{0}\right)-\frac{y^{2}}{2 \beta^{2}}+\log 2\right\} .
\end{aligned}
$$


3.4. Proof of Theorem 1.1. Finally, we conclude the proof of Theorem 1.1 by analyzing the optimization problem in Proposition 3.5 over $y$ and $\rho$.

ProOF. Fix $\rho \in \mathcal{P}$ such that $\sup _{y \in \mathbb{R}}\left[J(\rho, y)-H\left(\rho \mid \pi_{0}\right)\right]>-\infty$. This implies $J(\rho, y)>$ $-\infty$ for some $y$, so $\int_{-1}^{1} \operatorname{arctanh}(x)^{2} \rho(\mathrm{d} x)<\infty$. Furthermore, $H\left(\rho \mid \pi_{0}\right)<\infty$, so, in particular, $\rho(\mathrm{d} x)=f(x) \mathrm{d} x$ for some density function $f(x)$ on $(-1,1)$. Let $(q, \varphi, a, e)=$ $(Q(\rho), M(\rho), A(\rho), E(\rho))$. Then,

$$
\int \log g(x ; \varphi, q, y) \rho(\mathrm{d} x)=\chi_{1} y^{2}+\chi_{2} y+\chi_{3},
$$

where

$$
\begin{aligned}
\chi_{1}= & -\int \frac{x^{2}}{2 \beta^{2} q} \rho(\mathrm{d} x)=-\frac{1}{2 \beta^{2}}, \\
\chi_{2}= & \int \frac{x\left(\operatorname{arctanh}(x)-\beta \lambda \varphi+\beta^{2}(1-q) x\right)}{\beta^{2} q} \rho(\mathrm{d} x)=\frac{a}{\beta^{2} q}-\frac{\lambda}{\beta} \frac{\varphi^{2}}{q}+1-q, \\
\chi_{3}= & \int\left(\log \frac{1}{1-x^{2}}-\frac{\operatorname{arctanh}(x)^{2}}{2 \beta^{2} q}+\frac{\lambda \varphi \operatorname{arctanh}(x)}{\beta q}\right) \rho(\mathrm{d} x) \\
& +\frac{\left(2 \beta \lambda(1-q)-\lambda^{2}\right) \varphi^{2}-2 a(1-q)}{2 q}-\frac{\beta^{2}(1-q)^{2}}{2} .
\end{aligned}
$$

Let $\rho_{\varphi, q}$ be the law of $\tanh (z)$ where $z \sim \mathcal{N}\left(\beta \lambda \varphi, \beta^{2} q\right)$. Note that

$$
\begin{aligned}
& \int_{-1}^{1} \frac{1}{1-x^{2}} \exp \left(-\frac{\operatorname{arctanh}(x)^{2}}{2 \beta^{2} q}+\frac{\lambda \varphi \operatorname{arctanh}(x)}{\beta q}\right) \mathrm{d} x \\
& =\int_{\mathbb{R}} e^{-y^{2} /\left(2 \beta^{2} q\right)+\lambda \varphi y /(\beta q)} d y=e^{\lambda^{2} \varphi^{2} /(2 q)}\left(2 \pi \beta^{2} q\right)^{1 / 2},
\end{aligned}
$$

and

$$
h_{\varphi, q}(x)=e^{-\lambda^{2} \varphi^{2} /(2 q)}\left(2 \pi \beta^{2} q\right)^{-1 / 2} \frac{1}{1-x^{2}} \exp \left(-\frac{\operatorname{arctanh}(x)^{2}}{2 \beta^{2} q}+\frac{\lambda \varphi \operatorname{arctanh}(x)}{\beta q}\right)
$$

is the density function of the law $\rho_{\varphi, q}$. Then, $\chi_{3}=\int \log h_{\varphi, q}(x) \rho(\mathrm{d} x)+\frac{1}{2} \log \left(2 \pi \beta^{2} q\right)+\chi_{4}$ where

$$
\chi_{4}=\frac{\left(\beta \lambda \varphi^{2}-a\right)(1-q)}{q}-\frac{\beta^{2}(1-q)^{2}}{2}
$$

Note further that

$$
\sup _{y \in \mathbb{R}}\left[\left(\chi_{1}-\frac{1}{2 \beta^{2}}\right) y^{2}+\chi_{2} y\right]=\sup _{y \in \mathbb{R}}\left[-\frac{1}{\beta^{2}} y^{2}+\chi_{2} y\right]=\frac{\beta^{2} \chi_{2}^{2}}{4}
$$

and $H\left(\rho \mid \pi_{0}\right)=\log 2+\int_{-1}^{1} \log f(x) \rho(\mathrm{d} x)$, where $f(x)$ is the density of $\rho$. We hence obtain

$$
\begin{aligned}
\sup _{y \in \mathbb{R}} & {\left[J(\rho, y)-H\left(\rho \mid \pi_{0}\right)-y^{2} /\left(2 \beta^{2}\right)+\log 2\right] } \\
& =\frac{\beta^{2}(1-q)^{2}}{2}+\frac{\beta^{2} \chi_{2}^{2}}{4}+\chi_{4}-\int_{-1}^{1} \log \frac{f(x)}{h_{\varphi, q}(x)} \rho(\mathrm{d} x) \\
& =\frac{1}{4 \beta^{2}}\left(\beta^{2}(1-q)+\frac{\beta \lambda \varphi^{2}}{q}-\frac{a}{q}\right)^{2}-H\left(\rho \mid \rho_{\varphi, q}\right),
\end{aligned}
$$


where the second line simplifies $\beta^{2}(1-q)^{2} / 2+\beta^{2} \chi_{2}^{2} / 4+\chi_{4}$. Then, combining this with Corollary 3.4 and Proposition 3.5,

$$
\begin{aligned}
& \limsup n^{-1} \log \mathbb{E}\left[\operatorname{Crit}_{n}\left(U, V_{n}\right)\right] \\
& \quad \leq \sup _{(q, \varphi, a, e) \in U}\left[\frac{1}{4 \beta^{2}}\left(\beta^{2}(1-q)+\frac{\beta \lambda \varphi^{2}}{q}-\frac{a}{q}\right)^{2}-\inf _{\rho \in \mathcal{P}(q, \varphi, a, e)} H\left(\rho \mid \rho_{\varphi, q}\right)\right],
\end{aligned}
$$

where $\mathcal{P}(q, \varphi, a, e)=\{\rho \in \mathcal{P}:(Q(\rho), M(\rho), A(\rho), E(\rho))=(q, \varphi, a, e)\}$.

Recalling $u(q, a)$ from (1.12) and introducing Lagrange multipliers for these constraints, we have

$$
\begin{aligned}
\inf _{\rho \in \mathcal{P}(q, \varphi, a, e)} H\left(\rho \mid \rho_{\varphi, q}\right)= & \inf _{\rho \in \mathcal{P}} \sup _{\mu, \nu, \tau, \gamma} H\left(\rho \mid \rho_{\varphi, q}\right)-\mu(Q(\rho)-q)-v(M(\rho)-\varphi) \\
& -\tau(A(\rho)-a)-\gamma[u(Q(\rho), A(\rho))-E(\rho)-u(q, a)+e] \\
\geq & \sup _{\mu, \nu, \tau, \gamma} \mu q+v \varphi+\tau a+\gamma[u(q, a)-e]+\inf _{\rho \in \mathcal{P}} F(\rho ; \mu, \nu, \tau, \gamma),
\end{aligned}
$$

where

$$
\begin{aligned}
F & (\rho ; \mu, \nu, \tau, \gamma) \\
& =H\left(\rho \mid \rho_{\varphi, q}\right)-\mu Q(\rho)-v M(\rho)-\tau A(\rho)-\gamma[u(Q(\rho), A(\rho))-E(\rho)] \\
& =\int_{-1}^{1}\left(\log \frac{f(x)}{h_{\varphi, q}(x)}-\mu x^{2}-v x-\tau x \operatorname{arctanh}(x)+\frac{\gamma}{2}\left(\log \frac{1+x}{2}+\log \frac{1-x}{2}\right)\right) \rho(\mathrm{d} x) \\
& =H\left(\rho \mid \rho_{\mu, v, \tau, \gamma}\right)-\log I .
\end{aligned}
$$

Here,

$$
\begin{aligned}
\rho_{\mu, \nu, \tau, \gamma}(\mathrm{d} x)= & I^{-1} \exp \left(\mu x^{2}+v x+\tau x \operatorname{arctanh}(x)\right. \\
& \left.+\frac{\gamma}{2}\left(\log \frac{1+x}{2}+\log \frac{1-x}{2}\right)\right) h_{\varphi, q}(x) \mathrm{d} x
\end{aligned}
$$

is a suitably defined exponential family density with base measure $h_{\varphi, q}(x) \mathrm{d} x$, and $I$, as defined in (1.13), is the normalizing constant for this density. Thus

$$
\inf _{\rho \in \mathcal{P}} F(\rho ; \mu, \nu, \tau, \gamma)=-\log I
$$

with the infimum achieved at $\rho=\rho_{\mu, \nu, \tau, \gamma}$, and Theorem 1.1 follows.

4. Proof of Proposition 3.2. In this section we prove Proposition 3.2 which analyzes $\operatorname{det} \boldsymbol{H}_{n}(\boldsymbol{m})$. The following lemma is standard and can be found, for example, in [12], Lemma 11.

LEMMA 4.1. Let $\boldsymbol{W} \sim \operatorname{GOE}(n)$ and $\boldsymbol{x}, \boldsymbol{y} \in \mathbb{R}^{n}$. Denote by $\mathrm{P}_{\boldsymbol{x}}^{\perp}=\mathbf{I}-\boldsymbol{x} \boldsymbol{x}^{\top} /\|\boldsymbol{x}\|_{2}^{2}$ the projection orthogonal to $\boldsymbol{x}$. Then, we have

$$
\left.\boldsymbol{W}\right|_{\boldsymbol{W} x=y} \stackrel{d}{=} \mathrm{P}_{\boldsymbol{x}}^{\perp} \boldsymbol{W} \mathrm{P}_{\boldsymbol{x}}^{\perp}+\left(\boldsymbol{x} \boldsymbol{y}^{\top}+\boldsymbol{y} \boldsymbol{x}^{\top}\right) /\|\boldsymbol{x}\|_{2}^{2}-\boldsymbol{x} \boldsymbol{x}^{\top}\langle\boldsymbol{x}, \boldsymbol{y}\rangle /\|\boldsymbol{x}\|_{2}^{4} .
$$

Denoting $\boldsymbol{u} \equiv \boldsymbol{u}(\boldsymbol{m})=\operatorname{arctanh}(\boldsymbol{m})-\beta \lambda / n \cdot\langle\mathbf{1}, \boldsymbol{m}\rangle \mathbf{1}+\beta^{2}[1-Q(\boldsymbol{m})] \boldsymbol{m}$, the condition $\boldsymbol{g}_{n}(\boldsymbol{m})=\mathbf{0}$ is equivalent to $\boldsymbol{W} \boldsymbol{m}=\beta^{-1} \boldsymbol{u}$. Then, by equations (3.2)-(3.3), conditional on $\boldsymbol{g}_{n}(\boldsymbol{m})=\mathbf{0}$, the Hessian $\boldsymbol{H}_{n}(\boldsymbol{m})$ is equal in law to

$$
\boldsymbol{Z}(\boldsymbol{m})=\boldsymbol{D}(\boldsymbol{m})-\beta \boldsymbol{W}+\Delta(\boldsymbol{m}),
$$


where

$$
\boldsymbol{D}(\boldsymbol{m})=\operatorname{diag}\left(d_{1}, \ldots, d_{n}\right), \quad d_{i}=\frac{1}{1-m_{i}^{2}}+\beta^{2}[1-Q(\boldsymbol{m})]
$$

and

$$
\begin{aligned}
\Delta(\boldsymbol{m})= & \beta \boldsymbol{W} \boldsymbol{m} \boldsymbol{m}^{\top} /\|\boldsymbol{m}\|_{2}^{2}+\beta \boldsymbol{m} \boldsymbol{m}^{\top} \boldsymbol{W} /\|\boldsymbol{m}\|_{2}^{2}-\beta \boldsymbol{m} \boldsymbol{m}^{\top}\langle\boldsymbol{m}, \boldsymbol{W} \boldsymbol{m}\rangle /\|\boldsymbol{m}\|_{2}^{4} \\
& -\frac{\beta \lambda}{n} \cdot \mathbf{1 1}^{\top}-2 \beta^{2} / n \cdot \boldsymbol{m} \boldsymbol{m}^{\top}-\left(\boldsymbol{m} \boldsymbol{u}^{\top}+\boldsymbol{u} \boldsymbol{m}^{\top}\right) /\|\boldsymbol{m}\|_{2}^{2}+\boldsymbol{m} \boldsymbol{m}^{\top}\langle\boldsymbol{m}, \boldsymbol{u}\rangle /\|\boldsymbol{m}\|_{2}^{4} .
\end{aligned}
$$

Let $\sigma_{\beta}$ be the semicircle law with support $[-2 \beta, 2 \beta]$, let $\mu_{\boldsymbol{D}(\boldsymbol{m})}$ be the empirical spectral distribution of $\boldsymbol{D}(\boldsymbol{m})$ and define their additive free convolution [66]

$$
v_{\boldsymbol{m}}=\mu_{\boldsymbol{D}(\boldsymbol{m})} \boxplus \sigma_{\beta} .
$$

We expect $v_{\boldsymbol{m}}$ to approximate the bulk spectral distribution of $\boldsymbol{Z}(\boldsymbol{m})$ for large $n$ [56]. Denote $\mathbf{i}=\sqrt{-1}$, and let log denote the complex logarithm with branch cut on the negative real axis. For $\varepsilon>0$, let

$$
l_{\varepsilon}(x)=\log |x-\mathbf{i} \varepsilon|=\operatorname{Re} \log (x-\mathbf{i} \varepsilon) .
$$

Denote

$$
\operatorname{Tr} l_{\varepsilon}(\boldsymbol{Z})=\sum_{i=1}^{n} l_{\varepsilon}\left(\lambda_{i}(\boldsymbol{Z})\right)
$$

where $\lambda_{1}(\boldsymbol{Z}), \ldots, \lambda_{n}(\boldsymbol{Z})$ are the eigenvalues of $\boldsymbol{Z}$.

Recall $L(\boldsymbol{m})$ as defined in (3.5). We prove Proposition 3.2 via the following three lemmas. We defer the proofs of the first two to Sections 4.1 and 4.2 :

Lemma 4.2. Fix $\beta>0, \lambda \geq 0, \eta, b \in(0,1)$ and $a \in(0,1 / 9)$. Let $\boldsymbol{m}$ satisfy $\|\boldsymbol{m}\|_{\infty} \leq 1-$ $e^{-n^{b}}$ and $Q(\boldsymbol{m}) \in[\eta, 1]$, and set $\varepsilon=n^{-a}$. Then, there exist constants $C \equiv C(\beta, \lambda, \eta, b, a)>$ 0 and $n_{0} \equiv n_{0}(\beta, \lambda, \eta, b, a)>0$ such that, for all $n \geq n_{0}$,

$$
\mathbb{E}\left[\operatorname{Tr} l_{\varepsilon}(\boldsymbol{Z}(\boldsymbol{m}))\right] \leq n \int l_{\varepsilon}(x) v_{\boldsymbol{m}}(\mathrm{d} x)+C\left(\varepsilon^{-5}+n^{b}+\log n\right) .
$$

LEMMA 4.3. For any $\beta>0$ and $\boldsymbol{m} \in(-1,1)^{n}$, there exists a constant $C \equiv C(\beta)>0$ depending only on $\beta$ such that, for any $\varepsilon>0$,

$$
\int l_{\varepsilon}(x) v_{\boldsymbol{m}}(\mathrm{d} x) \leq L(\boldsymbol{m})+C \varepsilon
$$

LEMMA 4.4. For any $\varepsilon>0$, any $t>0$, any $\boldsymbol{m} \in(-1,1)^{n}$ and all $n \geq 1$,

$$
\mathbb{P}\left[\left|\operatorname{Tr} l_{\varepsilon}(\boldsymbol{Z}(\boldsymbol{m}))-\mathbb{E} \operatorname{Tr} l_{\varepsilon}(\boldsymbol{Z}(\boldsymbol{m}))\right| \geq n t\right] \leq 2 \exp \left(-n^{2} \varepsilon^{2} t^{2} /\left(8 \beta^{2}\right)\right) .
$$

Proof of Lemma 4.4. By [3], Lemma 2.3.1, if $f: \mathbb{R} \rightarrow \mathbb{R}$ is $L$-Lipschitz, then the map $\boldsymbol{Z} \mapsto \operatorname{Tr} f(\boldsymbol{Z})$ is $L \sqrt{2 n}$-Lipschitz with respect to the Frobenius norm of $\boldsymbol{Z}$. Then, for each fixed $\boldsymbol{m}$, the quantity $\operatorname{Tr} f(\boldsymbol{Z}(\boldsymbol{m}))$ is a $2 L \beta$-Lipschitz function of the $n(n-1) / 2$ standard Gaussian variables which parametrize $\mathrm{P}_{m}^{\perp} \boldsymbol{W} \mathrm{P}_{\boldsymbol{m}}^{\perp}$. By Gaussian concentration of measure, for any $t>0$, we have

$$
\mathbb{P}[|\operatorname{Tr} f(\boldsymbol{Z}(\boldsymbol{m}))-\mathbb{E} \operatorname{Tr} f(\boldsymbol{Z}(\boldsymbol{m}))| \geq n t] \leq 2 \exp \left(-\frac{n^{2} t^{2}}{8 L^{2} \beta^{2}}\right) .
$$


The result follows from observing that $l_{\varepsilon}$ is differentiable, with derivative at each $x \in \mathbb{R}$ satisfying

$$
\left|\frac{\mathrm{d}}{\mathrm{d} x} l_{\varepsilon}(x)\right|=\left|\frac{\mathrm{d}}{\mathrm{d} x} \operatorname{Re} \log (x-\mathbf{i} \varepsilon)\right|=\left|\operatorname{Re} \frac{1}{x-\mathbf{i} \varepsilon}\right| \leq \frac{1}{\varepsilon} .
$$

Proof of Proposition 3.2. Taking $\varepsilon=n^{-0.11}$, we obtain from Lemmas 4.2, 4.3 and 4.4 , for some constants $C, c, n_{0}>0$, all $n \geq n_{0}$ and all $t>0$,

$$
\mathbb{P}\left[\operatorname{Tr} l_{\varepsilon}(\boldsymbol{Z}(\boldsymbol{m})) \geq n L(\boldsymbol{m})+C n^{\max (0.89, b)}+n t\right] \leq 2 \exp \left(-c n^{1.78} t^{2}\right) .
$$

Then, setting $c_{n}=n L(\boldsymbol{m})+C n^{\max (0.89, b)}$,

$$
\begin{aligned}
\mathbb{E}[|\operatorname{det} \boldsymbol{Z}(\boldsymbol{m})|] & \leq \mathbb{E}\left[\exp \left(\operatorname{Tr} l_{\varepsilon}(\boldsymbol{Z}(\boldsymbol{m}))\right)\right] \\
& \leq \exp \left(c_{n}+n^{0.89}\right)+\int_{\exp \left(c_{n}+n^{0.89}\right)}^{\infty} \mathbb{P}\left[\exp \left(\operatorname{Tr} l_{\varepsilon}(\boldsymbol{Z}(\boldsymbol{m}))\right) \geq t\right] \mathrm{d} t \\
& =\exp \left(c_{n}+n^{0.89}\right)+e^{c_{n}} \int_{n^{0.89}}^{\infty} e^{s} \mathbb{P}\left[\operatorname{Tr} l_{\varepsilon}(\boldsymbol{Z}(\boldsymbol{m})) \geq c_{n}+s\right] \mathrm{d} s \\
& \leq \exp \left(c_{n}+n^{0.89}\right)+e^{c_{n}} \int_{n^{0.89}}^{\infty} 2 \exp \left(s-c s^{2} / n^{0.22}\right) \mathrm{d} s \\
& <\exp \left(n L(\boldsymbol{m})+C_{0} \cdot n^{\max (0.9, b)}\right),
\end{aligned}
$$

the last line holding for sufficiently large $n_{0}$ and $C_{0}$.

In the remainder of this section, we prove Lemmas 4.2 and 4.3.

4.1. Proof of Lemma 4.2. We first establish an analogue of Lemma 4.2 for a matrix of the form $\boldsymbol{D}-\beta \boldsymbol{W}$.

LEMMA 4.5. Fix $\beta>0$ and $a \in(0,1 / 9)$, and let $\varepsilon=n^{-a}$. Then, there exist constants $C \equiv C(\beta, a)>0$ and $n_{0} \equiv n_{0}(\beta, a)>0$ such that, for all $n \geq n_{0}$, the following holds: Let $\boldsymbol{D} \in \mathbb{R}^{n \times n}$ be any real symmetric matrix, let $\boldsymbol{H}=\boldsymbol{D}-\beta \boldsymbol{W}$ where $\boldsymbol{W} \sim \operatorname{GOE}(n)$ and let $v=\mu_{\boldsymbol{D}} \boxplus \sigma_{\beta}$. Then,

$$
\mathbb{E}\left[\operatorname{Tr} l_{\varepsilon}(\boldsymbol{H})\right] \leq n \int l_{\varepsilon}(x) v(\mathrm{~d} x)+C\left(\varepsilon^{-5}+\log \left(\|\boldsymbol{D}\|_{\text {op }}+1\right)+\log n\right) .
$$

By the results of [56], the Stieltjes transform

$$
g(z)=\int \frac{1}{x-z} v(\mathrm{~d} x)
$$

of the above measure $v$ satisfies, for each $z \in \mathbb{C}^{+}$, the fixed-point equation

$$
g(z)=\frac{1}{n} \sum_{i=1}^{n} \frac{1}{d_{i}-z-\beta^{2} g(z)}
$$

with $\left(d_{i}\right)_{i \leq n}$ denoting the eigenvalues of $\boldsymbol{D}$. We first provide a quantitative estimate of the approximation of the spectral law of $\boldsymbol{H}$ by $v$, in terms of their Stieltjes transforms at points $z \in \mathbb{C}^{+}$where $\operatorname{Im} z \gtrsim n^{-1 / 9}$. 
Lemma 4.6. Fix $\beta>0$, let $\boldsymbol{D} \in \mathbb{R}^{n \times n}$ be any real symmetric matrix and let $\boldsymbol{H}$ and $v$ be as in Lemma 4.5. For $z \in \mathbb{C}^{+}$, denote the Stieltjes transforms of $\boldsymbol{H}$ and $v$ as

$$
\hat{g}(z)=\frac{1}{n} \operatorname{Tr}(\boldsymbol{H}-z \mathbf{I})^{-1}, \quad g(z)=\int \frac{1}{x-z} v(\mathrm{~d} x) .
$$

If $n \geq 40 \max (\beta / \operatorname{Im} z, 1)^{9}$, then

$$
|\mathbb{E} \hat{g}(z)-g(z)|<\frac{12}{n \operatorname{Im} z} \max (\beta / \operatorname{Im} z, 1)^{5} .
$$

PROOF. We follow the approach of [57], Theorem 3.1, applying integration by parts and the Poincaré inequality. Fix $z \in \mathbb{C}^{+}$, and denote $\eta=\operatorname{Im} z$. Denote the resolvents of $\boldsymbol{H}$ and $\boldsymbol{D}$ as

$$
G_{\boldsymbol{H}}(z)=(\boldsymbol{H}-z \mathbf{I})^{-1}, \quad G_{\boldsymbol{D}}(z)=(\boldsymbol{D}-z \mathbf{I})^{-1} .
$$

Applying $\boldsymbol{A}^{-1}-\boldsymbol{B}^{-1}=\boldsymbol{A}^{-1}(\boldsymbol{B}-\boldsymbol{A}) \boldsymbol{B}^{-1}$, we obtain

$$
G_{\boldsymbol{H}}(z)-G_{\boldsymbol{D}}(z)=\beta G_{\boldsymbol{H}}(z) \boldsymbol{W} G_{\boldsymbol{D}}(z) .
$$

As $G_{D}(z)$ is deterministic, this yields

$$
\mathbb{E} G_{\boldsymbol{H}}(z)=G_{\boldsymbol{D}}(z)+\beta \mathbb{E}\left[G_{\boldsymbol{H}}(z) \boldsymbol{W}\right] G_{\boldsymbol{D}}(z) .
$$

We use integration by parts on the term $\mathbb{E}\left[G_{\boldsymbol{H}}(z) \boldsymbol{W}\right]$. For any differentiable bounded function $f: \mathbb{R} \rightarrow \mathbb{R}$, when $\xi \sim \mathcal{N}\left(0, \sigma^{2}\right)$,

$$
\mathbb{E}[\xi f(\xi)]=\sigma^{2} \mathbb{E}\left[f^{\prime}(\xi)\right] .
$$

Consider indices $i, j, k \in[n]$. If $j<k$, then setting $\boldsymbol{X}$ as the matrix with $(j, k)$ and $(k, j)$ entries equal to 1 and remaining entries 0 , we have

$$
\begin{aligned}
\frac{\partial G_{\boldsymbol{H}}(z)_{i j}}{\partial W_{j k}} & =\lim _{\delta \rightarrow 0} \delta^{-1}\left((\boldsymbol{H}-z \mathbf{I}-\beta \delta \boldsymbol{X})^{-1}-(\boldsymbol{H}-z \mathbf{I})^{-1}\right)_{i j} \\
& =\beta\left(G_{\boldsymbol{H}}(z) \boldsymbol{X} G_{\boldsymbol{H}}(z)\right)_{i j}=\beta\left(G_{\boldsymbol{H}}(z)_{i j} G_{\boldsymbol{H}}(z)_{k j}+G_{\boldsymbol{H}}(z)_{i k} G_{\boldsymbol{H}}(z)_{j j}\right) .
\end{aligned}
$$

For $z \in \mathbb{C}^{+}$with $\operatorname{Im} z=\eta$, we have $\left|G_{\boldsymbol{H}}(z)_{i j}\right| \leq 1 / \eta$. Then, (4.6) yields

$$
\mathbb{E}\left[G_{\boldsymbol{H}}(z)_{i j} W_{j k}\right]=\frac{1}{n} \mathbb{E}\left[\frac{\partial G_{\boldsymbol{H}}(z)_{i j}}{\partial W_{j k}}\right]=\frac{\beta}{n} \mathbb{E}\left[G_{\boldsymbol{H}}(z)_{i j} G_{\boldsymbol{H}}(z)_{k j}+G_{\boldsymbol{H}}(z)_{i k} G_{\boldsymbol{H}}(z)_{j j}\right] .
$$

Similar arguments yield the same identity when $j=k$. Then, applying $G_{\boldsymbol{H}}(z)_{k j}=G_{\boldsymbol{H}}(z)_{j k}$ and summing over $j$, we obtain

$$
\mathbb{E}\left[G_{\boldsymbol{H}}(z) \boldsymbol{W}\right]=\frac{\beta}{n} \mathbb{E}\left[G_{\boldsymbol{H}}(z)^{2}\right]+\beta \mathbb{E}\left[\hat{g}(z) G_{\boldsymbol{H}}(z)\right] .
$$

Denoting $\delta(z)=\hat{g}(z)-\mathbb{E} \hat{g}(z)$ and substituting the above into (4.5),

$$
\left(\mathbb{E} G_{\boldsymbol{H}}(z)\right)\left[\mathbf{I}-\beta^{2}(\mathbb{E} \hat{g}(z)) G_{\boldsymbol{D}}(z)\right]=\left(\mathbf{I}+\frac{\beta^{2}}{n} \mathbb{E}\left[G_{\boldsymbol{H}}(z)^{2}\right]+\beta^{2} \mathbb{E}\left[\delta(z) G_{\boldsymbol{H}}(z)\right]\right) G_{\boldsymbol{D}}(z) .
$$

Multiplying on the right by $G_{D}(z)^{-1}=\boldsymbol{D}-z \mathbf{I}$,

$$
\left(\mathbb{E} G_{\boldsymbol{H}}(z)\right)\left[\boldsymbol{D}-z \mathbf{I}-\beta^{2}(\mathbb{E} \hat{g}(z)) \mathbf{I}\right]=\mathbf{I}+\frac{\beta^{2}}{n} \mathbb{E}\left[G_{\boldsymbol{H}}(z)^{2}\right]+\beta^{2} \mathbb{E}\left[\delta(z) G_{\boldsymbol{H}}(z)\right] .
$$


Now, multiplying on the right by $\left[\boldsymbol{D}-z \mathbf{I}-\left(\beta^{2} \mathbb{E} \hat{g}(z)\right) \mathbf{I}\right]^{-1}=G_{\boldsymbol{D}}\left(z+\beta^{2} \mathbb{E} \hat{g}(z)\right)$, taking $n^{-1} \mathrm{Tr}$ and rearranging,

$$
\mathbb{E} \hat{g}(z)-\frac{1}{n} \sum_{i=1}^{n} \frac{1}{d_{i}-z-\beta^{2} \mathbb{E} \hat{g}(z)}=r(z),
$$

where

$$
\begin{aligned}
r(z)= & \frac{\beta^{2}}{n^{2}} \mathbb{E} \operatorname{Tr}\left(G_{\boldsymbol{H}}(z)^{2} G_{\boldsymbol{D}}\left(z+\beta^{2} \mathbb{E} \hat{g}(z)\right)\right) \\
& +\frac{\beta^{2}}{n} \mathbb{E}\left[\delta(z) \operatorname{Tr}\left(G_{\boldsymbol{H}}(z) G_{\boldsymbol{D}}\left(z+\beta^{2} \mathbb{E} \hat{g}(z)\right)\right)\right] \\
= & : \mathrm{I}+\mathrm{II} .
\end{aligned}
$$

Let us bound this remainder $r(z)$. Noting $\left\|(X-z \mathbf{I})^{-1}\right\|_{\text {op }} \leq 1 / \operatorname{Im} z$ for any real-symmetric $\boldsymbol{X}$, observing $\operatorname{Im} z+\beta^{2} \mathbb{E} \hat{g}(z) \geq \operatorname{Im} z$ and applying $|\operatorname{Tr} \boldsymbol{X}| \leq n\|\boldsymbol{X}\|_{\text {op }}$, we obtain

$$
|\mathrm{I}| \leq \frac{\beta^{2}}{n \eta^{3}}
$$

For II, applying these bounds and Cauchy-Schwarz,

$$
|\mathrm{II}| \leq \frac{\beta^{2}}{n} \mathbb{E}\left[|\delta(z)|^{2}\right]^{1 / 2} \frac{n}{\eta^{2}}=\frac{\beta^{2}}{\eta^{2}} \operatorname{Var}(\hat{g}(z))^{1 / 2},
$$

where $\operatorname{Var}(\hat{g})=\mathbb{E}\left[|\hat{g}-\mathbb{E} \hat{g}|^{2}\right]=\mathbb{E}\left[|\hat{g}|^{2}\right]-|\mathbb{E} \hat{g}|^{2}$. We apply the Poincaré inequality to bound $\operatorname{Var}(\hat{g}(z))$. For i.i.d. $\mathcal{N}(0,1)$ variables $\xi_{1}, \ldots, \xi_{k}$ and $f: \mathbb{R}^{k} \rightarrow \mathbb{C}$ with bounded partial derivatives,

$$
\operatorname{Var}\left(f\left(\xi_{1}, \ldots, \xi_{k}\right)\right) \leq \mathbb{E}\left[\left\|\nabla f\left(\xi_{1}, \ldots, \xi_{k}\right)\right\|_{2}^{2}\right]
$$

For $j<k$, a computation similar to (4.7) yields

$$
\begin{aligned}
& \frac{1}{\sqrt{n}} \frac{\partial \hat{g}(z)}{\partial W_{j k}}=\frac{1}{n^{3 / 2}} \sum_{i=1}^{n} \frac{\partial G_{\boldsymbol{H}}(z)_{i i}}{\partial W_{j k}}=\frac{2 \beta}{n^{3 / 2}}\left[G_{\boldsymbol{H}}(z)^{2}\right]_{j k}, \\
& \frac{1}{\sqrt{n / 2}} \frac{\partial \hat{g}(z)}{\partial W_{j j}}=\frac{\sqrt{2} \beta}{n^{3 / 2}}\left[G_{\boldsymbol{H}}(z)^{2}\right]_{j j} .
\end{aligned}
$$

Noting $\left(\sqrt{n / 2} W_{j j}: j \in[n]\right) \cup\left(\sqrt{n} W_{j k}: 1 \leq j<k \leq n\right)$ are i.i.d. $\mathcal{N}(0,1)$ variables, (4.9) yields

$$
\begin{aligned}
\operatorname{Var}(\hat{g}(z)) & \leq \mathbb{E}\left[\sum_{j=1}^{n} \frac{2 \beta^{2}}{n^{3}}\left|\left[G_{\boldsymbol{H}}(z)^{2}\right]_{j j}\right|^{2}+\sum_{j<k} \frac{4 \beta^{2}}{n^{3}}\left|\left[G_{\boldsymbol{H}}(z)^{2}\right]_{j k}\right|^{2}\right] \\
& =\frac{2 \beta^{2}}{n^{3}} \mathbb{E} \operatorname{Tr}\left(G_{\boldsymbol{H}}(z)^{2}{\overline{\left(G_{\boldsymbol{H}}(z)^{2}\right)}}^{\top}\right) \leq \frac{2 \beta^{2}}{n^{2} \eta^{4}} .
\end{aligned}
$$

Combining the above,

$$
|r(z)| \leq \frac{\beta^{2}}{n \eta^{4}}(\eta+\sqrt{2} \beta) .
$$


Finally, we compare (4.8) with the fixed-point equation (4.4) satisfied by $g(z)$ to obtain a bound on $\mathbb{E} \hat{g}(z)-g(z)$ : Denoting $(x)_{+}=\max (x, 0)$, we have

$$
\begin{aligned}
|r(z)|= & \left|\mathbb{E} \hat{g}(z)-g(z)-\frac{1}{n} \sum_{i=1}^{n}\left(\frac{1}{d_{i}-z-\beta^{2} \mathbb{E} \hat{g}(z)}-\frac{1}{d_{i}-z-\beta^{2} g(z)}\right)\right| \\
= & |\mathbb{E} \hat{g}(z)-g(z)|\left|1-\frac{1}{n} \sum_{i=1}^{n} \frac{\beta^{2}}{\left(d_{i}-z-\beta^{2} \mathbb{E} \hat{g}(z)\right)\left(d_{i}-z-\beta^{2} g(z)\right)}\right| \\
\geq & |\mathbb{E} \hat{g}(z)-g(z)| \\
& \times\left(1-\sqrt{\frac{1}{n} \sum_{i=1}^{n} \frac{\beta^{2}}{\left|d_{i}-z-\beta^{2} \mathbb{E} \hat{g}(z)\right|^{2}}} \sqrt{\left.\frac{1}{n} \sum_{i=1}^{n} \frac{\beta^{2}}{\left|d_{i}-z-\beta^{2} g(z)\right|^{2}}\right)_{+},}\right.
\end{aligned}
$$

the last step applying $|1-x| \geq(1-|x|)_{+}$and Cauchy-Schwarz. Taking the imaginary part of (4.4) and rearranging,

$$
\frac{\beta^{2} \operatorname{Im} g(z)}{\eta+\beta^{2} \operatorname{Im} g(z)}=\frac{1}{n} \sum_{i=1}^{n} \frac{\beta^{2}}{\left|d_{i}-z-\beta^{2} g(z)\right|^{2}} .
$$

We have

$$
\operatorname{Im} g(z)=\operatorname{Im} \int \frac{1}{x-z} v(\mathrm{~d} x)=\int \frac{\eta}{|x-z|^{2}} v(\mathrm{~d} x) \leq 1 / \eta
$$

and hence

$$
\frac{1}{n} \sum_{i=1}^{n} \frac{\beta^{2}}{\left|d_{i}-z-\beta^{2} g(z)\right|^{2}} \leq \frac{\beta^{2}}{\eta^{2}+\beta^{2}}
$$

Similarly, taking imaginary parts of (4.8) and rearranging,

$$
\frac{1}{n} \sum_{i=1}^{n} \frac{\beta^{2}}{\left|d_{i}-z-\beta^{2} \mathbb{E} \hat{g}(z)\right|^{2}}=\frac{\beta^{2} \operatorname{Im} \mathbb{E} \hat{g}(z)-\beta^{2} \operatorname{Im} r(z)}{\eta+\beta^{2} \operatorname{Im} \mathbb{E} \hat{g}(z)} \leq \frac{\beta^{2}}{\eta^{2}+\beta^{2}}+\frac{\beta^{2}|r(z)|}{\eta} .
$$

Applying $\sqrt{a+b} \leq \sqrt{a}+\sqrt{b}$ and the above bounds to (4.11), we obtain

$$
\begin{aligned}
|r(z)| & \geq|\mathbb{E} \hat{g}(z)-g(z)|\left(1-\frac{\beta^{2}}{\eta^{2}+\beta^{2}}-\sqrt{\frac{\beta^{2}}{\eta^{2}+\beta^{2}} \cdot \frac{\beta^{2}|r(z)|}{\eta}}\right)_{+} \\
& \geq|\mathbb{E} \hat{g}(z)-g(z)|\left(\frac{\eta^{2}}{\eta^{2}+\beta^{2}}-\sqrt{\frac{\beta^{2}|r(z)|}{\eta}}\right)_{+}
\end{aligned}
$$

When

$$
n \geq 40 \max \left(\frac{\beta}{\eta}, 1\right)^{9} \geq 20\left(\frac{\beta^{4}}{\eta^{4}}+\frac{\beta^{9}}{\eta^{9}}\right)>\frac{4 \beta^{4}(\eta+\sqrt{2} \beta)\left(\eta^{2}+\beta^{2}\right)^{2}}{\eta^{9}},
$$

we verify from (4.10) that $\sqrt{\beta^{2}|r(z)| / \eta} \leq \eta^{2} /\left(2\left(\eta^{2}+\beta^{2}\right)\right)$. Then, the above implies

$$
\begin{aligned}
|\mathbb{E} \hat{g}(z)-g(z)| & \leq \frac{2|r(z)|\left(\eta^{2}+\beta^{2}\right)}{\eta^{2}} \leq \frac{2 \beta^{2}(\eta+\sqrt{2} \beta)\left(\eta^{2}+\beta^{2}\right)}{n \eta^{6}} \\
& <\frac{6}{n \eta}\left(\frac{\beta^{2}}{\eta^{2}}+\frac{\beta^{5}}{\eta^{5}}\right) \leq \frac{12}{n \eta} \max \left(\frac{\beta}{\eta}, 1\right)^{5} .
\end{aligned}
$$


Proof of Lemma 4.5. Denote by $\hat{v}$ the empirical spectral measure of $\boldsymbol{H}$ and by $\hat{g}(z)$ its Stieltjes transform. Let $t_{n}=n\left(\|\boldsymbol{D}\|_{\text {op }}+\beta+1\right)$. Then, we have

$$
\begin{aligned}
\operatorname{Tr} l_{\varepsilon}(\boldsymbol{H}) & =n \int \operatorname{Re} \log (x-\mathbf{i} \varepsilon) \hat{v}(\mathrm{~d} x) \\
& =n \int \operatorname{Re}\left(\log \left(x-\mathbf{i} t_{n}\right)-\int_{\varepsilon}^{t_{n}} \frac{-\mathbf{i}}{x-\mathbf{i} t} \mathrm{~d} t\right) \hat{v}(\mathrm{~d} x) \\
& =n \int \log \left|x-\mathbf{i} t_{n}\right| \hat{v}(\mathrm{~d} x)+n \operatorname{Re}\left(\mathbf{i} \int_{\varepsilon}^{t_{n}} \hat{g}(\mathbf{i} t) \mathrm{d} t\right) .
\end{aligned}
$$

For $\varepsilon=n^{-a}$ where $a \in(0,1 / 9)$, we may apply Lemma 4.6 to get

$$
\begin{aligned}
\left|\int_{\varepsilon}^{t_{n}} \mathbb{E}[\hat{g}(\mathbf{i} t)] \mathrm{d} t-\int_{\varepsilon}^{t_{n}} g(\mathbf{i} t) \mathrm{d} t\right| & \leq \frac{12}{n}\left(\int_{\varepsilon}^{\beta} \frac{\beta^{5}}{t^{6}} \mathrm{~d} t+\int_{\beta}^{t_{n}} \frac{1}{t} \mathrm{~d} t\right) \\
& \leq C\left(\frac{\varepsilon^{-5}+\log \left(\|\boldsymbol{D}\|_{\mathrm{op}}+1\right)+\log n}{n}\right)
\end{aligned}
$$

for some $(\beta, a)$-dependent constants $C, n_{0}>0$ and all $n \geq n_{0}$. Reversing the above steps, we may write

$$
\operatorname{Re}\left(\mathbf{i} \int_{\varepsilon}^{t_{n}} g(\mathbf{i} t) \mathrm{d} t\right)=\int\left(-\log \left|x-\mathbf{i} t_{n}\right|+\log |x-\mathbf{i} \varepsilon|\right) v(\mathrm{~d} x),
$$

so combining the above yields

$$
\mathbb{E} \operatorname{Tr} l_{\varepsilon}(\boldsymbol{H}) \leq n \int l_{\varepsilon}(x) v(\mathrm{~d} x)+r_{1}+r_{2},
$$

where

$$
\begin{aligned}
& r_{1}=\mathbb{E}\left[n \int \log \left|x-\mathbf{i} t_{n}\right| \hat{v}(\mathrm{~d} x)\right]-n \int \log \left|x-\mathbf{i} t_{n}\right| v(\mathrm{~d} x), \\
& r_{2}=C\left(\varepsilon^{-5}+\log \left(\|\boldsymbol{D}\|_{\text {op }}+1\right)+\log n\right) .
\end{aligned}
$$

To bound $r_{1}$, note that, since $v=\mu_{\boldsymbol{D}} \boxplus \sigma_{\beta}$, we have $\sup \{|x|: x \in \operatorname{supp}(v)\} \leq\|\boldsymbol{D}\|_{\text {op }}+2 \beta$. Applying

$$
\log \left|x-\mathbf{i} t_{n}\right|-\log t_{n}=\operatorname{Re} \log \left(x-\mathbf{i} t_{n}\right)-\operatorname{Re} \log \left(-\mathbf{i} t_{n}\right)=\operatorname{Re} \frac{x}{\tilde{x}-\mathbf{i} t_{n}}
$$

for some $\tilde{x} \in \mathbb{R}$ with $|\tilde{x}| \leq|x|$, we obtain

$$
\left|\int \log \right| x-\mathbf{i} t_{n}\left|v(\mathrm{~d} x)-\log t_{n}\right| \leq \frac{\|\boldsymbol{D}\|_{\text {op }}+2 \beta}{t_{n}} \leq \frac{2}{n} .
$$

A similar argument holds for $\hat{v}$. Indeed, for all $t>0$, we have

$$
\mathbb{P}\left[\|\boldsymbol{H}\|_{\text {op }}>\|\boldsymbol{D}\|_{\text {op }}+2 \beta+t\right] \leq \mathbb{P}\left[\beta\|\boldsymbol{W}\|_{\text {op }}>2 \beta+t\right] \leq 2 \exp \left(-\frac{n t^{2}}{4 \beta^{2}}\right) ;
$$

see, for example, [29], Theorem II.11. Let $\mathcal{E}$ be the event where $\|\boldsymbol{H}\|_{\text {op }} \leq\|\boldsymbol{D}\|_{\text {op }}+2 \beta+1$. Then, we have

$$
\begin{aligned}
& S_{1}:=\left|\mathbb{E}\left[\int \log \left|x-\mathbf{i} t_{n}\right| \hat{v}(\mathrm{~d} x) \mid \mathcal{E}\right]-\log t_{n}\right| \leq \frac{\|\boldsymbol{D}\|_{\mathrm{op}}+2 \beta+1}{t_{n}} \leq \frac{2}{n}, \\
& S_{2}:=\mathbb{E}\left[\int \log \left|x-\mathbf{i} t_{n}\right| \hat{v}(\mathrm{~d} x) \mid \mathcal{E}^{c}\right] \leq \mathbb{E}\left[\log \left(\|\boldsymbol{H}\|_{\mathrm{op}}\right) \mid \mathcal{E}^{c}\right]+\log \left(t_{n}\right) .
\end{aligned}
$$


Note that, by (4.14),

$$
\begin{aligned}
\mathbb{E} & {\left[\log \left(\|\boldsymbol{H}\|_{\text {op }}\right) \mid \mathcal{E}^{c}\right] \mathbb{P}\left[\mathcal{E}^{c}\right] } \\
& =\mathbb{E}\left[\log \left(\|\boldsymbol{H}\|_{\text {op }}\right) \mathbf{1}\left\{\mathcal{E}^{c}\right\}\right] \\
& =\int_{0}^{\infty} \mathbb{P}\left[\|\boldsymbol{H}\|_{\text {op }} \geq \max \left(e^{t},\|\boldsymbol{D}\|_{\text {op }}+2 \beta+1\right)\right] \mathrm{d} t \\
& \leq \log \left(\|\boldsymbol{D}\|_{\text {op }}+2 \beta+1\right) \cdot 2 e^{-n /\left(4 \beta^{2}\right)}+\int_{\log \left(\|\boldsymbol{D}\|_{\text {op }}+2 \beta+1\right)}^{\infty} \mathbb{P}\left[\|\boldsymbol{H}\|_{\text {op }}>e^{t}\right] \mathrm{d} t \\
& <\left[\log \left(\|\boldsymbol{D}\|_{\text {op }}+1\right)+\log n\right] \cdot e^{-c n}
\end{aligned}
$$

for a constant $c \equiv c(\beta)>0$ and $n \geq n_{0}(\beta)>0$. Then,

$$
\begin{aligned}
\left|\mathbb{E} \int \log \right| x-\mathbf{i} t_{n}\left|\hat{v}(\mathrm{~d} x)-\log t_{n}\right| & \leq S_{1} \cdot \mathbb{P}[\mathcal{E}]+\left[S_{2}+\log \left(t_{n}\right)\right] \cdot \mathbb{P}\left[\mathcal{E}^{c}\right] \\
& \leq \frac{2}{n}+\left[\log \left(\|\boldsymbol{D}\|_{\text {op }}+1\right)+\log n\right] \cdot e^{-c n} .
\end{aligned}
$$

Combining this bound with equation (4.13), we obtain $\left|r_{1}\right| \leq \log \left(\|\boldsymbol{D}\|_{\text {op }}+1\right)+\log n$ for sufficiently large $n_{0}$ and all $n \geq n_{0}$. Then, the lemma follows from (4.12).

Proof of Lemma 4.2. Fix $\boldsymbol{m}$ and denote $\boldsymbol{H}=\boldsymbol{D}-\beta \boldsymbol{W}$, so that $\boldsymbol{Z}=\boldsymbol{H}+\Delta$. Lemma 4.5 gives

$$
\mathbb{E}\left[\operatorname{Tr} l_{\varepsilon}(\boldsymbol{H})\right] \leq n \int l_{\varepsilon}(x) v(\mathrm{~d} x)+C\left(\varepsilon^{-5}+\log \left(\|\boldsymbol{D}\|_{\text {op }}+1\right)+\log n\right) .
$$

From (4.2) the operator norm of $\Delta$ can be bounded by

$$
\begin{aligned}
\|\Delta\|_{\mathrm{op}}= & \left\|\beta \boldsymbol{W} \boldsymbol{m} \boldsymbol{m}^{\top} /\right\| \boldsymbol{m}\left\|_{2}^{2}\right\|_{\mathrm{op}}+\left\|\beta \boldsymbol{m} \boldsymbol{m}^{\top} \boldsymbol{W} /\right\| \boldsymbol{m}\left\|_{2}^{2}\right\|_{\mathrm{op}} \\
& +\left\|\beta \boldsymbol{m} \boldsymbol{m}^{\top}\langle\boldsymbol{m}, \boldsymbol{W} \boldsymbol{m}\rangle /\right\| \boldsymbol{m}\left\|_{2}^{4}\right\|_{\mathrm{op}}+\left\|\beta \lambda / n \cdot \mathbf{1 1}^{\top}\right\|_{\mathrm{op}}+\left\|2 \beta^{2} / n \cdot \boldsymbol{m} \boldsymbol{m}^{\top}\right\|_{\mathrm{op}} \\
& +\left\|\left(\boldsymbol{m} \boldsymbol{u}^{\top}+\boldsymbol{u} \boldsymbol{m}^{\top}\right) /\right\| \boldsymbol{m}\left\|_{2}^{2}\right\|_{F}+\left\|\boldsymbol{m} \boldsymbol{m}^{\top}\langle\boldsymbol{m}, \boldsymbol{u}\rangle /\right\| \boldsymbol{m}\left\|_{2}^{4}\right\|_{F} \\
\leq & 3 \beta\|\boldsymbol{W}\|_{\mathrm{op}}+2 \beta^{2}+\beta \lambda+3\|\boldsymbol{u}\|_{2} /\|\boldsymbol{m}\|_{2} .
\end{aligned}
$$

For $Q(\boldsymbol{m}) \in[\eta, 1]$ and $\boldsymbol{m} \in\left[-1+e^{-n^{b}}, 1-e^{-n^{b}}\right]$, we have

$$
\begin{aligned}
\|\boldsymbol{u}\|_{2} & \leq\|\operatorname{arctanh}(\boldsymbol{m})\|_{2}+\|\beta \lambda / n \cdot\langle\mathbf{1}, \boldsymbol{m}\rangle \mathbf{1}\|_{2}+\left\|\beta^{2}(1-Q(\boldsymbol{m})) \boldsymbol{m}\right\|_{2} \\
& \leq \sqrt{n}\|\operatorname{arctanh}(\boldsymbol{m})\|_{\infty}+\left(\beta^{2}+\beta \lambda\right) \sqrt{n} \leq \sqrt{n}\left(n^{b}+\left(\beta^{2}+\beta \lambda\right)\right)
\end{aligned}
$$

and $\|\boldsymbol{m}\|_{2} \geq \sqrt{n \eta}$. Accordingly, we have

$$
\|\Delta\|_{\text {op }} \leq 3 \lambda\|\boldsymbol{W}\|_{\text {op }}+\left(2 \beta^{2}+\beta \lambda\right)+3\left(n^{b}+\left(\beta^{2}+\beta \lambda\right)\right) / \sqrt{\eta} .
$$

Note that $\Delta$ is given in equation (4.2) which has rank at most 8. Suppose it has $r_{+}$positive eigenvalues and $r_{-}$negative eigenvalues, where $r_{+}+r_{-} \leq 8$ (it is possible that $r_{+}$or $r_{-}$ is zero). Denote the eigenvalues of $\boldsymbol{H}$ as $\lambda_{1}(\boldsymbol{H}) \geq \lambda_{2}(\boldsymbol{H}) \geq \cdots \geq \lambda_{k}(\boldsymbol{H}) \geq 0>\lambda_{k+1}(\boldsymbol{H}) \geq$ $\cdots \geq \lambda_{n}(\boldsymbol{H})$ and those of $\boldsymbol{Z}$ as $\lambda_{1}(\boldsymbol{Z}) \geq \lambda_{2}(\boldsymbol{Z}) \geq \cdots \geq \lambda_{n}(\boldsymbol{Z})$. We apply the following bounds (we use the convention that, if $k=0$, the set $\{1,2, \ldots, k\}=\varnothing$ ):

- For $i \in S \equiv\left\{1, \ldots, r_{+}\right\} \cup\left\{n+1-r_{-}, \ldots, n\right\} \cup\left\{k-r_{-}+1, \ldots, k+r_{+}\right\}$, we have

$$
\left|\lambda_{i}(\boldsymbol{Z})\right| \leq\|\boldsymbol{H}\|_{\text {op }}+\|\Delta\|_{\text {op }}
$$


- The rest of the eigenvalues of $\boldsymbol{Z}$ satisfy, by Weyl's eigenvalue interlacing,

$$
\begin{array}{ll}
\lambda_{i-r_{+}}(\boldsymbol{H}) \geq \lambda_{i}(\boldsymbol{Z}) \geq 0, & i \in\left\{r_{+}+1, r_{+}+2, \ldots, k-r_{-}\right\}, \\
\lambda_{i+r_{-}}(\boldsymbol{H}) \leq \lambda_{i}(\boldsymbol{Z}) \leq 0, & i \in\left\{k+r_{+}+1, k+r_{+}+2, \ldots, n-r_{-}\right\} .
\end{array}
$$

Then,

$$
\begin{aligned}
\operatorname{Tr} l_{\varepsilon}(\boldsymbol{Z}) & \leq \operatorname{Tr} l_{\varepsilon}(\boldsymbol{H})-\sum_{i \in S} l_{\varepsilon}\left(\lambda_{i}(\boldsymbol{H})\right)+16 \log \left(\|\boldsymbol{H}\|_{\text {op }}+\|\Delta\|_{\text {op }}\right) \\
& \leq \operatorname{Tr} l_{\varepsilon}(\boldsymbol{H})-16 \log \varepsilon+16 \log \left(\|\boldsymbol{D}\|_{\text {op }}+\lambda\|\boldsymbol{W}\|_{\text {op }}+\|\Delta\|_{\text {op }}\right) .
\end{aligned}
$$

Note that for $\boldsymbol{m}$ with $\|\boldsymbol{m}\|_{\infty} \leq 1-e^{-n^{b}}$, we have $\|\boldsymbol{D}\|_{\text {op }} \leq e^{2 n^{b}}+\beta^{2}$. Then, taking expectations of the above and applying Jensen's inequality, the result follows from equations (4.15) and (4.16), and $\mathbb{E}\left[\|\boldsymbol{W}\|_{\text {op }}\right] \leq 3$.

4.2. Proof of Lemma 4.3. Fix $\lambda>0$ and $\boldsymbol{m} \in(-1,1)^{n}$, and write as shorthand $v \equiv v_{m}$. Lemma 4.3 follows from several properties of $v$ and its support.

LEMMA 4.7. The Stieltjes transform $g(z)$ of $v$ extends continuously from $\mathbb{C}^{+}$to $\mathbb{C}^{+} \cup \mathbb{R}$. Denoting this extension also by $g(z)$, the measure $v$ admits a continuous density given by

$$
f(z)=\frac{1}{\pi} \operatorname{Im} g(z), \quad z \in \mathbb{R} .
$$

At each $z \in \mathbb{R}$, letting $z_{0} \in \mathbb{R}$ denote the closest point where $f\left(z_{0}\right)=0$, we have

$$
f(z) \leq\left(\frac{3}{4 \pi^{3} \beta}\left|z-z_{0}\right|\right)^{1 / 3} .
$$

Proof. See [14], Corollaries 1, 2, 5.

LEMMA 4.8. The support of $v$ is contained in $\left[0, d_{\max }+2 \beta\right]$, where $d_{\max }=\max _{i \leq n} d_{i}$.

Proof. We note that (4.4) holds also for $z \in \mathbb{R}$ by continuity, where $g(z)$ for $z \in \mathbb{R}$ is the continuous extension defined in Lemma 4.7. Fix any $z \leq 0$, and consider the function

$$
F(g)=\frac{1}{n} \sum_{i=1}^{n} \frac{1}{d_{i}-z-\beta^{2} g}
$$

defining the right side of (4.4). Let $p_{1}<\cdots<p_{k}$ be the distinct values among

$$
\left\{\left(d_{i}-z\right) / \beta^{2}: i=1, \ldots, n\right\} .
$$

Then, $F(g)$ is a rational function with poles $p_{1}, \ldots, p_{k}$.

The equation $g=F(g)$ is rearranged as a polynomial equation of degree $k+1$, and hence it is as most $k+1$ complex roots counting multiplicity. From the graph of $F(g)$, there is at least one real root between $p_{j}$ and $p_{j+1}$ for each $j=1, \ldots, k-1$. Furthermore, when $z \leq 0$, we have $p_{1}>1-Q(\boldsymbol{m})$ and

$$
F(1-Q(\boldsymbol{m}))=\frac{1}{n} \sum_{i=1}^{n} \frac{1}{\left(1-m_{i}^{2}\right)^{-1}-z} \leq \frac{1}{n} \sum_{i=1}^{n}\left(1-m_{i}^{2}\right)=1-Q(\boldsymbol{m}) .
$$

Then, from the graph of $F(g)$ on $g \in\left(0, p_{1}\right)$, the equation $g=F(g)$ has at least two real roots in this interval, counting multiplicity. So all $k+1$ roots of $g=F(g)$ are real; there is exactly one root in each interval $\left(p_{j}, p_{j+1}\right)$ and exactly two roots in $\left(0, p_{1}\right)$. In particular, $g(z)$ is real. Then, by Lemma 4.7 the density of $v$ is 0 for all $z \leq 0$, so $v$ is supported on $[0, \infty)$. The upper bound $d_{\max }+2 \beta$ follows from $\sup \{|x|: x \in \operatorname{supp}(v)\} \leq\|\boldsymbol{D}\|_{\text {op }}+2 \beta$, as $v=\mu_{\boldsymbol{D}} \boxplus \sigma_{\beta}$. 
LEMMA 4.9. At $z=0$, the Stieltjes transform $g(0)$ is the smallest real root of (4.4) and satisfies $0<g(0) \leq 1-Q(\boldsymbol{m})$. Furthermore, for $L(\boldsymbol{m})$ defined in (3.5), we have

$$
\int \log (x) v(\mathrm{~d} x) \leq L(\boldsymbol{m}) .
$$

PROOF. From the proof of Lemma 4.8, the equation $g=F(g)$ has exactly one root $g \in$ $\left(p_{j}, p_{j+1}\right)$ for each $j=1, \ldots, k-1$ and exactly two roots $g \in\left(0, p_{1}\right)$ counting multiplicity. From the graph of $F$, this implies $F^{\prime}(g)>1$ for each root $g \in\left(p_{j}, p_{j+1}\right)$, and also the two roots $g_{1} \leq g_{2}$ in $\left(0, p_{1}\right)$ satisfy $F^{\prime}\left(g_{1}\right) \leq 1$ and $F^{\prime}\left(g_{2}\right) \geq 1$, with $F^{\prime}\left(g_{2}\right)=1$ if and only if $g_{1}=g_{2}$. At any $z<0$, since $z \notin \operatorname{supp}(v), g(z)$ is analytic in a neighborhood of $z$ and is given by (4.3). Differentiating (4.3) in $z$, we verify $g^{\prime}(z)>0$. On the other hand, differentiating (4.4) in $z$, we obtain

$$
g^{\prime}(z)=\frac{1}{n} \sum_{i=1}^{n} \frac{1+\beta^{2} g^{\prime}(z)}{\left(d_{i}-z-\beta^{2} g(z)\right)^{2}},
$$

so the condition $g^{\prime}(z)>0$ implies

$$
1-\frac{1}{n} \sum_{i=1}^{n} \frac{\beta^{2}}{\left(d_{i}-z-\beta^{2} g(z)\right)^{2}}>0 .
$$

Thus, $F^{\prime}(g(z))<1$. This holds for all $z<0$, so we must have $F^{\prime}(g(0)) \leq 1$ at $z=0$ by continuity. Then, $g(0)$ is the smallest real root to $g=F(g)$ at $z=0$. Since $1-Q(\boldsymbol{m})$ is one such root by (4.18), we have $0<g(0) \leq 1-Q(\boldsymbol{m})$.

To prove the bound on $\int \log (x) v(\mathrm{~d} x)$, we write, for any $T>0$,

$$
\begin{aligned}
\int \log (x) v(\mathrm{~d} x) & =\int\left(\log (x-\mathbf{i} T)-\int_{0}^{T} \frac{-\mathbf{i}}{x-\mathbf{i} t} \mathrm{~d} t\right) v(\mathrm{~d} x) \\
& =\int \log (x-\mathbf{i} T) v(\mathrm{~d} x)+\mathbf{i} \int_{0}^{T} g(\mathbf{i} t) \mathrm{d} t
\end{aligned}
$$

For each $t \in(0, T)$, applying (4.4), we have

$$
\begin{aligned}
g(\mathbf{i} t) & =g(\mathbf{i} t)\left(1+\beta^{2} g^{\prime}(\mathbf{i} t)\right)-\beta^{2} g(\mathbf{i} t) g^{\prime}(\mathbf{i} t) \\
& =\frac{1}{n} \sum_{i=1}^{n} \frac{1+\beta^{2} g^{\prime}(\mathbf{i} t)}{d_{i}-\mathbf{i} t-\beta^{2} g(\mathbf{i} t)}-\beta^{2} g(\mathbf{i} t) g^{\prime}(\mathbf{i} t) .
\end{aligned}
$$

Then, $-\mathbf{i} g(\mathbf{i} t)=B^{\prime}(t)$ for

$$
B(t):=\frac{1}{n} \sum_{i=1}^{n} \log \left(d_{i}-\mathbf{i} t-\beta^{2} g(\mathbf{i} t)\right)+\frac{\beta^{2}}{2} g(\mathbf{i} t)^{2},
$$

so $\mathbf{i} \int_{0}^{T} g(\mathbf{i} t) \mathrm{d} t=B(0)-B(T)$. We next take $T \rightarrow \infty$, using $|g(\mathbf{i} T)| \leq \sup _{x}(1 /|x-i T|) \leq$ $1 / T$ from equation (4.3). Applying a Taylor expansion of $\log (x-\mathbf{i} T)$, we have

$$
\int \log (x-\mathbf{i} T) v(\mathrm{~d} x)=\log (-\mathbf{i} T)+O(1 / T), \quad B(T)=\log (-\mathbf{i} T)+O(1 / T) .
$$

(In the first equation we use the fact that $v$ has bounded support; cf. Lemma 4.8, and in the second we use the definition of $B$, together with $|g(\mathbf{i} T)| \leq 1 / T$.) Combining the above, we obtain $\int \log (x) v(\mathrm{~d} x)=B(0)$. 
Finally, note that for $F$ as defined in the proof of Lemma 4.8, the equation $g=F(g)$ is equivalent to $0=R^{\prime}(g)$ where

$$
R(g)=\frac{\beta^{2} g^{2}}{2}+\frac{1}{n} \sum_{i=1}^{n} \log \left(d_{i}-\beta^{2} g\right) .
$$

Recall that either $g(0)=1-Q(\boldsymbol{m})$ or $g(0)<1-Q(\boldsymbol{m})$, and these are the two roots of this equation $0=R^{\prime}(g)$ in $\left(-\infty, p_{1}\right)$. Since $R(g) \rightarrow \infty$ when $g \rightarrow-\infty$, we obtain in both cases $B(0)=R(g(0)) \leq R(1-Q(\boldsymbol{m}))=L(\boldsymbol{m})$.

Proof of Lemma 4.3. For any $x>0$,

$$
\begin{aligned}
l_{\varepsilon}(x)-\log x & =\operatorname{Re} \log (x-\mathbf{i} \varepsilon)-\operatorname{Re} \log (x) \\
& =\log \sqrt{x^{2}+\varepsilon^{2}}-\log x=\frac{\sqrt{x^{2}+\varepsilon^{2}}-x}{x+\tilde{\varepsilon}}
\end{aligned}
$$

for some $\tilde{\varepsilon} \in\left[0, \sqrt{x^{2}+\varepsilon^{2}}-x\right]$. Then, $\left|l_{\varepsilon}(x)-\log x\right| \leq \varepsilon / x$, so

$$
\int l_{\varepsilon}(x) v(\mathrm{~d} x) \leq \int \log (x) v(\mathrm{~d} x)+\varepsilon \int(1 / x) v(\mathrm{~d} x) .
$$

The result follows from Lemma 4.9, Lemma 4.8 and the density decay condition (4.17) at the smallest edge of $v$ in the case where $0 \in \operatorname{supp}(v)$.

5. Proof of Theorem 1.2. We now prove Theorem 1.2 on the Bayes risk achieved by minimizing the TAP free energy in the $\mathbb{Z}_{2}$ synchronization model, corresponding to $\beta=\lambda$.

Throughout the proof we write $\mathcal{F}$ as shorthand for $\mathcal{F}_{\lambda, \lambda}$. Let $q_{\star}$ be the largest real solution of the equation

$$
q_{\star}=\mathbb{E}_{G}\left[\tanh \left(\lambda^{2} q_{\star}+\sqrt{\lambda^{2} q_{\star}} G\right)^{2}\right],
$$

for $G \sim \mathcal{N}(0,1)$. Note that, when $\lambda>1$, we have $q_{\star}>0$ [32], Lemma 4.2. Denote

$$
\begin{aligned}
& \varphi_{\star}=q_{\star}, \quad a_{\star}=\lambda^{2} q_{\star}, \\
& e_{\star}=-\frac{\lambda^{2}}{4}\left(1-2 q_{\star}-q_{\star}^{2}\right)-\mathbb{E}_{G}\left\{\log \left[2 \cosh \left(\lambda^{2} q_{\star}+\sqrt{\lambda^{2} q_{\star}} G\right)\right]\right\},
\end{aligned}
$$

and recall $Q(\boldsymbol{m})=\|\boldsymbol{m}\|_{2}^{2} / n$ and $M(\boldsymbol{m})=\langle\boldsymbol{x}, \boldsymbol{m}\rangle / n$. Recall $\mathcal{C}_{\lambda, n}$ the set of critical points of $\mathcal{F}$ with energy below $-\lambda^{2} / 3$, and define

$$
\mathcal{C}_{\lambda, n}^{+} \equiv\left\{\boldsymbol{m} \in[-1,1]^{n}: \nabla \mathcal{F}(\boldsymbol{m})=\mathbf{0}, \mathcal{F}(\boldsymbol{m}) \leq-\lambda^{2} / 3, M(\boldsymbol{m}) \geq 0\right\}
$$

so that $\mathcal{C}_{\lambda, n}=\mathcal{C}_{\lambda, n}^{+} \cup-\mathcal{C}_{\lambda, n}^{+}$.

5.1. Localization of critical points. Denote $\Gamma_{\varepsilon} \subseteq[-1,1]^{n}$ to be the preimage of $(Q, M, \mathcal{F})$ at a neighborhood of $\left(q_{\star}, \varphi_{\star}, e_{\star}\right)$ :

$$
\Gamma_{\varepsilon} \equiv\left\{\boldsymbol{m} \in[-1,1]^{n}:\left|Q(\boldsymbol{m})-q_{\star}\right| \leq \varepsilon,\left|M(\boldsymbol{m})-\varphi_{\star}\right| \leq \varepsilon,\left|\mathcal{F}(\boldsymbol{m})-e_{\star}\right|<\varepsilon\right\} .
$$

We first prove that $\varnothing \neq \mathcal{C}_{\lambda, n}^{+} \subseteq \Gamma_{\varepsilon}$ with high probability.

Proposition 5.1. There exists $\lambda_{0}<\infty$ such that, for any $\lambda \geq \lambda_{0}$ and $\varepsilon>0$, for some constants $n_{0} \equiv n_{0}(\lambda, \varepsilon)$ and $c_{0} \equiv c_{0}(\lambda, \varepsilon)$, with probability at least $1-e^{-c_{0} n}$ for all $n \geq n_{0}$, we have: $\mathcal{C}_{\lambda, n}^{+} \subseteq \Gamma_{\varepsilon}$, and some global minimizer $\boldsymbol{m}_{\star}$ of $\mathcal{F}$ satisfies $\boldsymbol{m}_{\star} \in \mathcal{C}_{\lambda, n}^{+} \subseteq \Gamma_{\varepsilon}$. 
Recall that in Theorem 1.1 , for $\lambda>0$, choosing $\beta=\lambda$, taking $\eta, b \in(0,1)$ and taking closed sets $U \subseteq[\eta, 1] \times \mathbb{R}^{3}, V_{n}=\left[-1+e^{-n^{b}}, 1-e^{-n^{b}}\right]$, we have

$$
\limsup _{n \rightarrow \infty} n^{-1} \log \mathbb{E}\left[\operatorname{Crit}_{n}\left(U, V_{n}\right)\right] \leq \sup _{(q, \varphi, a, e) \in U} S_{\star}(q, \varphi, a, e) .
$$

To prove Proposition 5.1, the following first bounds the function $S_{\star}(q, \varphi, a, e)$ for $(q, \varphi)$ in a small neighborhood of $\left(q_{\star}, \varphi_{\star}\right)$. We defer its proof to Section 6 .

Proposition 5.2. For any $\lambda>0$, we have

$$
S_{\star}\left(q_{\star}, \varphi_{\star}, a_{\star}, e_{\star}\right)=0 .
$$

Furthermore, there exist constants $c_{0}, \lambda_{0}>0$ and a function $c(\lambda)>0$ such that, for any $\lambda \geq \lambda_{0}$ and any $(q, \varphi)$ satisfying $\left|q-q_{\star}\right|,\left|\varphi-\varphi_{\star}\right| \leq c_{0} / \lambda^{2}$, we have

$$
S_{\star}(q, \varphi, a, e) \leq-c(\lambda)\left[\left(a-a_{\star}\right)^{2}+\left(q-q_{\star}\right)^{2}+\left(\varphi-\varphi_{\star}\right)^{2}+\left(e-e_{\star}\right)^{2}\right] .
$$

Note that, for $c_{0}, \lambda_{0}$ as above and $\lambda \geq \lambda_{0}$, this implies

$$
\sup \left\{S_{\star}(q, \varphi, a, e):\left|q-q_{\star}\right|,\left|\varphi-\varphi_{\star}\right| \leq c_{0} / \lambda^{2},(a, e) \in \mathbb{R}^{2}\right\} \leq 0,
$$

with the supremum uniquely attained at $\left(q_{\star}, \varphi_{\star}, a_{\star}, e_{\star}\right)$. The following then localizes any critical point of $\mathcal{F}$ satisfying Theorem 5.1 to the above neighborhood of $\left(q_{\star}, \varphi_{\star}\right)$. Its proof is in Appendix C.

Proposition 5.3. Fix any positive integer $k$. Then, there exist $\lambda_{0}, C_{0}>0$ and functions $c_{0}(\lambda), n_{0}(\lambda)>0$ such that, for all $\lambda \geq \lambda_{0}$ and $n \geq n_{0}(\lambda)$ with probability at least $1-e^{-c_{0}(\lambda) n}$, any $\boldsymbol{m} \in \mathcal{C}_{\lambda, n}^{+}$also satisfies $Q(\boldsymbol{m}), M(\boldsymbol{m}) \geq 1-C_{0} / \lambda^{k}$.

Finally, the following shows that, with high probability, there are no critical points with any coordinate outside the set $V_{n}$ in Theorem 1.1 .

LEMMA 5.4. There exist constants $n_{0}$, and $c_{0}>0$ so that, for $n \geq n_{0}$ with probability at least $1-e^{-c_{0} n}$, all points $\boldsymbol{m} \in(-1,1)^{n}$ satisfying $\boldsymbol{g}_{n}(\boldsymbol{m})=\mathbf{0}$ also satisfy

$$
\|\boldsymbol{m}\|_{\infty} \leq 1-e^{-4 \lambda^{2}-6 \lambda \sqrt{n}}
$$

PROOF. Note that, when $\boldsymbol{g}_{n}(\boldsymbol{m})=\mathbf{0}$, we have

$$
\begin{aligned}
\|\operatorname{arctanh}(\boldsymbol{m})\|_{\infty} & =\left\|-\lambda^{2} / n \cdot\langle\mathbf{1}, \boldsymbol{m}\rangle \mathbf{1}-\lambda \cdot \boldsymbol{W} \boldsymbol{m}+\lambda^{2}[1-Q(\boldsymbol{m})] \boldsymbol{m}\right\|_{\infty} \\
& \leq 2 \lambda^{2}+\lambda\|\boldsymbol{W}\|_{\text {op }} \sqrt{n} .
\end{aligned}
$$

With probability at least $1-e^{-c_{0} n}$ for $n \geq n_{0}$ and some $n_{0}>0$ and $c_{0}>0$, we have $\|\boldsymbol{W}\|_{\text {op }} \leq$ 3 , and hence $\|\operatorname{arctanh}(\boldsymbol{m})\|_{\infty} \leq 2 \lambda^{2}+3 \lambda \sqrt{n}$. Applying $\tanh (x) \leq 1-e^{-2 x}$ for $x \geq 0$, with probability at least $1-e^{-c_{0} n}$, we have

$$
\|\boldsymbol{m}\|_{\infty} \leq \tanh \left(\|\operatorname{arctanh}(\boldsymbol{m})\|_{\infty}\right) \leq 1-e^{-4 \lambda^{2}-6 \lambda \sqrt{n}}
$$

Proof of Proposition 5.1. According to Lemma 5.4 and Proposition 5.3, there exist constants $\lambda_{0}, C_{0}$ and functions $c_{0}(\lambda), n_{0}(\lambda)$ such that, for $\lambda \geq \lambda_{0}$ and $n \geq n_{0}(\lambda)$, the following happens with probability at least $1-e^{-c_{0} n}$ : For any $\boldsymbol{m} \in \mathcal{C}_{\lambda, n}^{+}$, we have $\|\boldsymbol{m}\|_{\infty} \leq 1-e^{-4 \lambda^{2}-6 \lambda \sqrt{n}} \leq 1-e^{-\lambda^{2} \sqrt{n}}$ and $Q(\boldsymbol{m}), M(\boldsymbol{m}) \geq 1-C_{0} / \lambda^{3}$. 
Define

$$
\begin{aligned}
U_{\varepsilon}= & \left\{(q, \varphi, a, e):(q, \varphi) \in\left[1-C_{0} / \lambda^{3}, 1\right]^{2},(a, e) \in \mathbb{R}^{2}\right\} \\
& \cap\left\{(q, \varphi, a, e): \max \left\{\left|q-q_{\star}\right|,\left|\varphi-\varphi_{\star}\right|,\left|e-e_{\star}\right|\right\} \geq \varepsilon\right\}
\end{aligned}
$$

and $V_{n}=\left[-1+e^{-\lambda^{2} \sqrt{n}}, 1-e^{-\lambda^{2} \sqrt{n}}\right]$. According to Theorem 1.1, we have

$$
\limsup _{n \rightarrow \infty} n^{-1} \log \mathbb{E}\left[\operatorname{Crit}_{n}\left(U_{\varepsilon}, V_{n}\right)\right] \leq \sup _{(q, \varphi, a, e) \in U_{\varepsilon}} S_{\star}(q, \varphi, a, e) .
$$

According to Proposition 5.2, for $\lambda \geq \lambda_{0}$ with $\lambda_{0}$ large enough, we have

$$
0>\sup _{(q, \varphi, a, e) \in U_{\varepsilon}} S_{\star}(q, \varphi, a, e) \equiv-s_{0}(\lambda, \varepsilon) .
$$

Therefore, there exists $n_{0}(\lambda, \varepsilon)$ large enough so that, for $n \geq n_{0}(\lambda, \varepsilon)$,

$$
n^{-1} \log \mathbb{E}\left[\operatorname{Crit}_{n}\left(U_{\varepsilon}, V_{n}\right)\right] \leq-s_{0}(\lambda, \varepsilon) / 2
$$

Accordingly, by Markov's inequality, we have

$$
\mathbb{P}\left(\operatorname{Crit}_{n}\left(U_{\varepsilon}, V_{n}\right) \geq 1\right) \leq \mathbb{E}\left[\operatorname{Crit}_{n}\left(U_{\varepsilon}, V_{n}\right)\right] \leq \exp \left\{-s_{0} n / 2\right\} .
$$

Combining the above statements concludes that $\mathcal{C}_{\lambda, n}^{+} \subseteq \Gamma_{\varepsilon}$.

Next, we show that a global minimizer $\boldsymbol{m}_{\star} \in \mathcal{C}_{\lambda, n}^{+}$. The gradient of $\mathcal{F}$ diverges at the boundary of $[-1,1]^{n}$ and points outside $[-1,1]^{n}$ because of the entropy term. Hence, any global minimizer $\boldsymbol{m}_{\star}$ belongs to $(-1,1)^{n}$ and satisfies $\nabla \mathcal{F}\left(\boldsymbol{m}_{\star}\right)=\mathbf{0}$. Furthermore, note $\mathcal{F}(\boldsymbol{x})=$ $-\lambda^{2} / 2-\lambda\langle\boldsymbol{x}, \boldsymbol{W} \boldsymbol{x}\rangle /(2 n) \leq-\lambda^{2} / 2+\lambda\|\boldsymbol{W}\|_{\text {op }} / 2$, and $\mathbb{P}\left(\|\boldsymbol{W}\|_{\text {op }} \leq 3\right) \geq 1-e^{-c_{0} n}$ for $n \geq n_{0}$ with some constant $c_{0}$ and $n_{0}$. Accordingly, we have $\mathcal{F}\left(\boldsymbol{m}_{\star}\right) \leq \mathcal{F}(\boldsymbol{x}) \leq-\lambda^{2} / 2+3 \lambda / 2 \leq$ $-\lambda^{2} / 3$ for $\lambda \geq 9$ and $n \geq n_{0}$ with probability at least $1-e^{-c_{0} n}$. Taking $-\boldsymbol{m}_{\star}$ in place of $\boldsymbol{m}_{\star}$ if necessary, we ensure $M\left(\boldsymbol{m}_{\star}\right) \geq 0$.

5.2. Proof of Theorem 1.2. In this regime, [32] showed that $\operatorname{MMSE}_{n}(\lambda)$ converges to a limiting value which admits the following characterization.

PROPOSITION 5.5 ([32]). Let $\lambda>0$ be a fixed constant. Let $q_{\star}$ be the largest nonnegative solution to the equation

$$
q_{\star}=\mathbb{E}_{G \sim \mathcal{N}(0,1)}\left[\tanh \left(\lambda^{2} q_{\star}+\sqrt{\lambda^{2} q_{\star}} G\right)^{2}\right] .
$$

Then, $\lim _{n \rightarrow \infty} \operatorname{MMSE}_{n}(\lambda)=1-q_{\star}^{2}$. The value $q_{\star}$ is monotonically increasing in $\lambda$, with $q_{\star}=0$ when $\lambda \leq 1$ and $q_{\star} \in(0,1)$ when $\lambda>1$.

Proof of Proposition 5.5. See [32], equations (167) and (143) and Lemma 4.2. (The notational identification with [32] is $\gamma_{*} / \lambda \leftrightarrow q_{\star}$ and $\lambda \leftrightarrow \lambda^{2}$. [32] establishes this result with additional direct observations under a binary erasure channel with erasure probability $1-\varepsilon$, and the statement for $\varepsilon=0$ follows from continuity in $\varepsilon$.)

Proof OF TheOREM 1.2. Note that $\mathcal{C}_{\lambda, n}$ is compact. Hence, when $\mathcal{C}_{\lambda, n} \neq \varnothing$, the maximum value of $\left\|\boldsymbol{m} \boldsymbol{m}^{\top}-\widehat{\boldsymbol{X}}_{\text {Bayes }}\right\|_{F}^{2}$ over $\mathcal{C}_{\lambda, n}$ can be attained by some $\boldsymbol{m} \in[-1,1]^{n}$. We denote $\boldsymbol{m}_{\star} \in \mathbb{R}^{n}$ to be a random element within the set $\arg \max _{\boldsymbol{m} \in \mathcal{C}_{\lambda, n}}\left\|\boldsymbol{m} \boldsymbol{m}^{\top}-\widehat{\boldsymbol{X}}_{\text {Bayes }}\right\|_{F}^{2}$ if $\mathcal{C}_{\lambda, n} \neq \varnothing$, or $\boldsymbol{m}_{\star}=\mathbf{0}$ if $\mathcal{C}_{\lambda, n}=\varnothing$. Since $\mathcal{C}_{\lambda, n}=\mathcal{C}_{\lambda, n}(\boldsymbol{Y})$ is uniquely defined in terms of $\mathcal{F}_{\lambda, \beta}$, which itself depends in a measurable way on $\boldsymbol{Y}$ (see equation (1.6)), it follows that $\boldsymbol{m}_{\star}$ can be taken to be a measurable function of $\boldsymbol{Y}$. (Measurability follows from [21], Theorem 1, using the fact that $\left\{(\boldsymbol{m}, \boldsymbol{Y}): \boldsymbol{m} \in \mathcal{C}_{\lambda, n}(\boldsymbol{Y})\right\}$ is Borel.) In particular, $\boldsymbol{m}_{\star}$ is independent of $\boldsymbol{x}$ conditional on $\boldsymbol{Y}$. 
By Proposition 5.1, for any constants $\varepsilon, \delta>0$, for sufficiently large $n$, with probability at least $1-\delta$, we have $\mathcal{C}_{\lambda, n} \neq \varnothing$ and

$$
n^{-1}\left\|\boldsymbol{m}_{\star}\right\|^{2} \in\left[q_{\star}-\varepsilon, q_{\star}+\varepsilon\right], \quad n^{-1}\left|\left\langle\boldsymbol{m}_{\star}, \boldsymbol{x}\right\rangle\right| \in\left[q_{\star}-\varepsilon, q_{\star}+\varepsilon\right] .
$$

Denoting the above good event to be $\mathcal{E}$, we have

$$
\begin{aligned}
n^{-2} \mathbb{E}\left[\left\|\boldsymbol{m}_{\star} \boldsymbol{m}_{\star}^{\top}-\boldsymbol{x} \boldsymbol{x}^{\top}\right\|_{F}^{2} \mid \mathcal{E}\right] & =n^{-2} \mathbb{E}\left[\|\boldsymbol{x}\|^{4}+\left\|\boldsymbol{m}_{\star}\right\|^{4}-2\left|\left\langle\boldsymbol{m}_{\star}, \boldsymbol{x}\right\rangle\right|^{2} \mid \mathcal{E}\right] \\
& \leq\left(1+\left(q_{\star}+\varepsilon\right)^{2}-2\left(q_{\star}-\varepsilon\right)^{2}\right) \leq 1-q_{\star}^{2}+6 \varepsilon,
\end{aligned}
$$

so that

$$
\begin{aligned}
n^{-2} \mathbb{E}\left[\left\|\boldsymbol{m}_{\star} \boldsymbol{m}_{\star}^{\top}-\boldsymbol{x} \boldsymbol{x}^{\top}\right\|_{F}^{2}\right] & \leq n^{-2} \mathbb{E}\left[\left\|\boldsymbol{m}_{\star} \boldsymbol{m}_{\star}^{\top}-\boldsymbol{x} \boldsymbol{x}^{\top}\right\|_{F}^{2} \mid \mathcal{E}\right] \mathbb{P}(\mathcal{E})+2 \cdot \mathbb{P}\left(\mathcal{E}^{c}\right) \\
& \leq 1-q_{\star}^{2}+6 \varepsilon+2 \delta
\end{aligned}
$$

As $\boldsymbol{m}_{\star}$ is a measurable function $\boldsymbol{Y}$, orthogonanality of $\left(\boldsymbol{x} \boldsymbol{x}^{\top}-\widehat{\boldsymbol{X}}_{\text {Bayes }}\right)=\left(\boldsymbol{x} \boldsymbol{x}^{\top}-\mathbb{E}\left[\boldsymbol{x} \boldsymbol{x}^{\top} \mid \boldsymbol{Y}\right]\right)$ with respect to any function measurable on $\boldsymbol{Y}$, implies

$$
n^{-2} \mathbb{E}\left[\left\|\boldsymbol{m}_{\star} \boldsymbol{m}_{\star}^{\top}-\boldsymbol{x} \boldsymbol{x}^{\top}\right\|_{F}^{2}\right]=n^{-2} \mathbb{E}\left[\left\|\boldsymbol{m}_{\star} \boldsymbol{m}_{\star}^{\top}-\widehat{\boldsymbol{X}}_{\text {Bayes }}\right\|_{F}^{2}\right]+\operatorname{MMSE}_{n}(\lambda) .
$$

Then, Proposition 5.5 implies, for all $n \geq n_{0}(\lambda, \varepsilon, \delta)$,

$$
n^{-2} \mathbb{E}\left[\left\|\boldsymbol{m}_{\star} \boldsymbol{m}_{\star}^{\top}-\widehat{\boldsymbol{X}}_{\text {Bayes }}\right\|_{F}^{2}\right]<7 \varepsilon+2 \delta .
$$

Then, by the definition of $\boldsymbol{m}_{\star}$, we have

$$
\left(n^{-2} \sup _{\boldsymbol{m} \in \mathcal{C}_{\lambda, n}}\left\|\boldsymbol{m} \boldsymbol{m}^{\top}-\widehat{\boldsymbol{X}}_{\text {Bayes }}\right\|_{F}^{2}\right) \mathbf{1}\left\{\mathcal{C}_{\lambda, n} \neq \varnothing\right\} \leq n^{-2}\left\|\boldsymbol{m}_{\star} \boldsymbol{m}_{\star}^{\top}-\widehat{\boldsymbol{X}}_{\text {Bayes }}\right\|_{F}^{2},
$$

so that (1.20) holds.

6. Proof of Proposition 5.2. Denote $\xi \sim \mathcal{N}\left(\lambda^{2} \varphi_{\star}, \lambda^{2} q_{\star}\right)$. Note that $\varphi_{\star}=q_{\star}$ implies $\mathbb{E}\{f(-\xi)\}=\mathbb{E}\left\{e^{-2 \xi} f(\xi)\right\}$ whence $\mathbb{E}\left\{\tanh (\xi)^{2}\right\}=\mathbb{E}\{\tanh (\xi)\}$. Applying Gaussian integration by parts, we may verify from (5.7) and (5.2) that

$$
\begin{aligned}
\varphi_{\star} & =\mathbb{E}[\tanh (\xi)], \quad q_{\star}=\mathbb{E}\left[\tanh ^{2}(\xi)\right], \quad a_{\star}=\mathbb{E}[\xi \tanh (\xi)], \\
u\left(q_{\star}, a_{\star}\right)-e_{\star} & =\mathbb{E}[\log 2 \cosh (\xi)] .
\end{aligned}
$$

Note that

$$
S\left(q_{\star}, \varphi_{\star}, a_{\star}, e_{\star} ; \mu, \nu, \tau, \gamma\right)=-q_{\star} \mu-\varphi_{\star} \nu-a_{\star} \tau-\left[u\left(q_{\star}, a_{\star}\right)-e_{\star}\right] \gamma+\log I,
$$

where

$$
I=\mathbb{E}\left[\exp \left(\mu \tanh ^{2}(\xi)+v \tanh (\xi)+\tau \xi \tanh (\xi)+\gamma \log 2 \cosh (\xi)\right)\right] .
$$

Then, $S\left(q_{\star}, \varphi_{\star}, a_{\star}, e_{\star} ; \mu, v, \tau, \gamma\right)$ is a convex function of $(\mu, v, \tau, \gamma)$, with derivative in $(\mu, \nu, \tau, \gamma)$ equal to 0 at $(\mu, v, \tau, \gamma)=0$. Thus, $S_{\star}\left(q_{\star}, \varphi_{\star}, a_{\star}, e_{\star}\right)=0$ follows.

In the remainder of this section, we establish equation (5.4). Note that $q_{\star} \rightarrow 1$ as $\lambda \rightarrow \infty$ (see, e.g., [32], Lemma 3.2). Throughout the proof we assume $\lambda_{0}$ is large enough such that the conditions of the proposition guarantee $(q, \varphi) \in[1 /(1+\varepsilon), 1]^{2}$ for $\varepsilon \equiv 0.01$. Denote

$$
\begin{aligned}
S_{+}(q, \varphi, a) & =\frac{1}{4 \lambda^{2} q^{2}}\left[a-\lambda^{2} \varphi^{2}-\lambda^{2}(1-q) q\right]^{2}, \\
S_{-}(q, \varphi, a, e ; \mu, \nu, \tau, \gamma) & =-q \mu-\varphi \nu-a \tau-[u(q, a)-e] \gamma+\log I,
\end{aligned}
$$


so that $S=S_{+}+S_{-}$and

$$
S_{\star}(q, \varphi, a, e)=S_{+}(q, \varphi, a)+\inf _{\mu, \nu, \tau, \gamma} S_{-}(q, \varphi, a, e ; \mu, \nu, \tau, \gamma) .
$$

Denote $Q(x)=\tanh ^{2} x, M(x)=\tanh x, A(x)=x \tanh x$ and $U(x)=\log [2 \cosh (x)]$.

Step 1. Upper bound $S_{+}$. Noting $a_{\star}=\lambda^{2} \varphi_{\star}^{2}+\lambda^{2} q_{\star}\left(1-q_{\star}\right)$, we have

$$
S_{+}(q, \varphi, a)=\frac{1}{4 \lambda^{2} q^{2}}\left\{\left(a-a_{\star}\right)-\left(\lambda^{2} \varphi^{2}-\lambda^{2} \varphi_{\star}^{2}\right)-\left[\lambda^{2} q(1-q)-\lambda^{2} q_{\star}\left(1-q_{\star}\right)\right]\right\}^{2} .
$$

By the inequality

$$
\left(x_{1}+x_{2}+x_{3}\right)^{2} \leq(1+\varepsilon)^{2} x_{1}^{2}+(2+2 / \varepsilon)^{2} x_{2}^{2}+(2+2 / \varepsilon)^{2} x_{3}^{2}
$$

and the conditions $q, \varphi \in[1 /(1+\varepsilon), 1]$ and $\varepsilon=0.01$, we have

$$
\begin{aligned}
S_{+}(q, \varphi, a) \leq & (1+\varepsilon)^{2}\left(a-a_{\star}\right)^{2} /\left(4 \lambda^{2} q^{2}\right) \\
& +(2+2 / \varepsilon)^{2} \lambda^{4}\left\{\left(\varphi^{2}-\varphi_{\star}^{2}\right)^{2}+\left[q(1-q)-q_{\star}\left(1-q_{\star}\right)\right]^{2}\right\} /\left(4 \lambda^{2} q^{2}\right) \\
\leq & (1+\varepsilon)^{4}\left(a-a_{\star}\right)^{2} /\left(4 \lambda^{2}\right)+\left(10 / \varepsilon^{2}\right) \lambda^{2}\left(\varphi-\varphi_{\star}\right)^{2}+\left(10 / \varepsilon^{2}\right) \lambda^{2}\left(q-q_{\star}\right)^{2} .
\end{aligned}
$$

Step 2. Bound the moment generating function of $Q, M, A$ and $U$. Let $x \sim \mathcal{N}\left(\lambda^{2} \varphi, \lambda^{2} q\right)$. Let $\mathbb{E}$ and Var be taken with respect to the randomness of $x$. We bound $\mathbb{E}\left[e^{\mu(Q-\mathbb{E} Q)}\right]$, $\mathbb{E}\left[e^{\nu(M-\mathbb{E} M)}\right], \mathbb{E}\left[e^{\tau(A-\mathbb{E} A)}\right]$ and $\mathbb{E}\left[e^{\gamma(U-\mathbb{E} U)}\right]$.

Since $|Q| \leq 1$, we have, for $|\mu|<1$,

$$
\begin{aligned}
& \mathbb{E}[\exp \{\mu(Q-\mathbb{E} Q)\}] \\
& \quad \leq 1+\frac{1}{2} \mu^{2} \operatorname{Var}(Q)+\sum_{k=3}^{\infty} \frac{1}{k !}|\mu|^{k} \mathbb{E}\left[|Q-\mathbb{E} Q|^{k}\right] \\
& \quad \leq 1+\frac{1}{2} \mu^{2} \operatorname{Var}(Q) \sum_{k=2}^{\infty}|\mu|^{k-2} \leq \exp \left\{\mu^{2} \operatorname{Var}(Q) /[2(1-|\mu|)]\right\}
\end{aligned}
$$

Similarly, we have, for $|\nu|<1$,

$$
\mathbb{E}[\exp \{v(M-\mathbb{E} M)\}] \leq \exp \left\{v^{2} \operatorname{Var}(M) /[2(1-|v|)]\right\} .
$$

To bound $\mathbb{E}\left[e^{\tau(A-\mathbb{E} A)}\right]$, note that $A(x)=x \tanh (x)$ is $L_{a}$-Lipschitz. Indeed, simple calculus shows that $\sup _{t} A^{\prime}(t)=A^{\prime}\left(A^{-1}(1)\right)=A^{-1}(1)$, whence $L_{a}=A^{-1}(1) \leq 1.2$. Applying Gaussian concentration of measure (see, e.g., [18], Theorem 5.5), we have

$$
\mathbb{E}[\exp \{\tau(A-\mathbb{E} A)\}] \leq \exp \left\{\lambda^{2} q \tau^{2} L_{a}^{2} / 2\right\} \leq \exp \left\{\lambda^{2} \tau^{2} L_{a}^{2} / 2\right\} .
$$

Similarly, to bound $\mathbb{E}\left[e^{\gamma(U-\mathbb{E} U)}\right]$, note $U(x)=\log [2 \cosh (x)]$ is 1 -Lipschitz. Hence, we have

$$
\mathbb{E}[\exp \{\gamma(U-\mathbb{E} U)\}] \leq \exp \left\{\lambda^{2} q \gamma^{2} / 2\right\} \leq \exp \left\{\lambda^{2} \gamma^{2} / 2\right\}
$$

Step 3. Upper bound $S_{-}$. Set $\alpha=1+\varepsilon$ and $\kappa=(3+3 \varepsilon) / \varepsilon$, where $\varepsilon=0.01$ as above. By Holder's inequality,

$$
\begin{aligned}
& \mathbb{E}\left[e^{\mu(Q-\mathbb{E} Q)+v(M-\mathbb{E} M)+\tau(A-\mathbb{E} A)+\gamma(U-\mathbb{E} U)}\right] \\
& \quad \leq \mathbb{E}\left[e^{\alpha \tau(A-\mathbb{E} A)}\right]^{1 / \alpha} \mathbb{E}\left[e^{\kappa \mu(Q-\mathbb{E} Q)}\right]^{1 / \kappa} \mathbb{E}\left[e^{\kappa \nu(M-\mathbb{E} M)}\right]^{1 / \kappa} \mathbb{E}\left[e^{\kappa \gamma(U-\mathbb{E} U)}\right]^{1 / \kappa} .
\end{aligned}
$$


Given estimates (6.3), (6.4), (6.5) and (6.6), we have, for $|\mu|,|\nu|<1 / \kappa$,

$$
\begin{aligned}
\mathbb{E} & {\left[e^{\mu(Q-\mathbb{E} Q)+v(M-\mathbb{E} M)+\tau(A-\mathbb{E} A)+\gamma(U-\mathbb{E} U)}\right] } \\
& \leq \exp \left\{\frac{\alpha \tau^{2} \lambda^{2} L_{a}^{2}}{2}+\frac{\kappa \mu^{2} \operatorname{Var}(Q)}{2(1-\kappa|\mu|)}+\frac{\kappa \nu^{2} \operatorname{Var}(M)}{2(1-\kappa|v|)}+\frac{\kappa \gamma^{2} \lambda^{2}}{2}\right\},
\end{aligned}
$$

and hence

$$
\begin{aligned}
S_{-} & (q, \varphi, a, e ; \mu, \nu, \tau, \gamma) \\
\leq & -\tau(a-\mathbb{E}[A])+\frac{\alpha \tau^{2} \lambda^{2} L_{a}^{2}}{2}-\mu(q-\mathbb{E}[Q])+\frac{\kappa \mu^{2} \operatorname{Var}(Q)}{2(1-\kappa|\mu|)} \\
& -v(\varphi-\mathbb{E}[M])+\frac{\kappa v^{2} \operatorname{Var}(M)}{2(1-\kappa|v|)}-\gamma(u(q, a)-e-\mathbb{E}[U])+\frac{\kappa \gamma^{2} \lambda^{2}}{2} .
\end{aligned}
$$

To bound $\operatorname{Var}(Q)$ and $\operatorname{Var}(M)$, note that $\tanh (x) \geq 1-e^{-x}$. Then, for $(q, \varphi) \in[1 /(1+$ $\varepsilon), 1]^{2}$ with $\varepsilon=0.01$, there exists a universal constant $\lambda_{0}$ such that, when $\lambda \geq \lambda_{0}$, we have (denoting $\phi(x)=\mathbb{P}(|G| \geq x)$ for $G \sim \mathcal{N}(0,1)$ )

$$
\begin{aligned}
\mathbb{E}[1-\tanh x] & =\mathbb{E}\left[(1-\tanh x) \mathbf{1}\left\{x \geq \lambda^{2} / 2\right\}\right]+\mathbb{E}\left[(1-\tanh x) \mathbf{1}\left\{x<\lambda^{2} / 2\right\}\right] \\
& \leq e^{-\lambda^{2} / 2}+2 \mathbb{P}\left(x<\lambda^{2} / 2\right) \leq e^{-\lambda^{2} / 2}+\phi(\lambda(\varphi-1 / 2)) \leq e^{-\lambda^{2} / 5}
\end{aligned}
$$

and hence

$$
\operatorname{Var}(M)=\mathbb{E}\left[\tanh ^{2} x\right]-\mathbb{E}[\tanh x]^{2} \leq(1+\mathbb{E}[\tanh x])(1-\mathbb{E}[\tanh x]) \leq e^{-\lambda^{2} / 10} .
$$

Similarly, $\operatorname{Var}(Q) \leq e^{-\lambda^{2} / 10}$.

Now, we take $L_{q}^{2} \geq e^{-\lambda^{2} / 10}$ to be determined, and we take $\tau, \mu, v, \gamma$ to be

$$
\tau=\frac{a-\mathbb{E}[A]}{\alpha \lambda^{2} L_{a}^{2}}, \quad \mu=\frac{q-\mathbb{E}[Q]}{2 \kappa L_{q}^{2}}, \quad \nu=\frac{\varphi-\mathbb{E}[M]}{2 \kappa L_{q}^{2}}, \quad \gamma=\frac{u(q, a)-e-\mathbb{E}[U]}{\kappa \lambda^{2}} .
$$

Then, as long as

$$
\max \{|q-\mathbb{E}[Q]|,|\varphi-\mathbb{E}[M]|\} \leq L_{q}^{2},
$$

we have $|\mu|,|\nu| \leq 1 /(2 \kappa)$, and, according to equation (6.7), we have

$$
\begin{aligned}
& \inf _{\mu, \nu, \tau, \gamma} S_{-}(q, \varphi, a, e ; \mu, v, \tau, \gamma) \\
& \quad \leq-\frac{(a-\mathbb{E}[A])^{2}}{2 \alpha \lambda^{2} L_{a}^{2}}-\frac{(q-\mathbb{E}[Q])^{2}}{4 \kappa L_{q}^{2}}-\frac{(\varphi-\mathbb{E}[M])^{2}}{4 \kappa L_{q}^{2}}-\frac{(u(q, a)-e-\mathbb{E}[U])^{2}}{2 \kappa \lambda^{2}} .
\end{aligned}
$$

Step 4. Bound $\mathbb{E} A-a_{\star}, \mathbb{E} Q-q_{\star}, \mathbb{E} M-\varphi_{\star}$ and $\mathbb{E} U-\left(u\left(q_{\star}, a_{\star}\right)-e_{\star}\right)$. Recall that the expectations in (6.10) are with respect to $x \sim \mathcal{N}\left(\lambda^{2} \varphi, \lambda^{2} q\right)$ while the quantities of (6.1) are defined with $\xi \sim \mathcal{N}\left(\lambda^{2} \varphi_{\star}, \lambda^{2} q_{\star}\right)$. To bound this difference, define $D_{F, \#}=$ $\left.\sup _{q, \varphi \in[0.9,1]^{2}} \mid(\mathrm{d} / \mathrm{d} \#) \mathbb{E}_{G}\left[F\left(\lambda^{2} \varphi+\sqrt{\lambda^{2} q} G\right)\right]\right) \mid$ for $F=A, Q, M, U$ and $\#=q, \varphi$, where $G \sim \mathcal{N}(0,1)$. Now, we bound $D_{F}, \#$. We denote $x=\lambda^{2} \varphi+\sqrt{\lambda^{2} q} G$, where (to simplify notation) in each line below $\varphi, q \geq 0.9$ are chosen to maximize the corresponding expression. Applying (6.8) and Gaussian integration by parts, we obtain

$$
\begin{aligned}
& D_{A, \varphi} \leq|(\mathrm{d} / \mathrm{d} \varphi) \mathbb{E}[x \tanh x]|=\lambda^{2}\left|\mathbb{E}\left[\tanh x+x\left(1-\tanh ^{2} x\right)\right]\right| \leq 2 \lambda^{2}, \\
& D_{M, \varphi} \leq|(\mathrm{d} / \mathrm{d} \varphi) \mathbb{E}[\tanh x]| \leq \lambda^{2} \mathbb{E}\left[1-\tanh ^{2} x\right] \leq 2 \lambda^{2} e^{-\lambda^{2} / 5} .
\end{aligned}
$$


Similar arguments, omitted for brevity, show

$$
\begin{aligned}
& D_{A, q}, D_{U, \varphi} \leq \lambda^{2}, \quad D_{M, q} \leq 2 \lambda^{2} e^{-\lambda^{2} / 5}, \\
& D_{Q, \varphi}, D_{Q, q} \leq 4 \lambda^{2} e^{-\lambda^{2} / 5}, \quad D_{U, q} \leq \lambda^{2} e^{-\lambda^{2} / 5} .
\end{aligned}
$$

Moreover, denoting $D_{u, q}$ as the Lipschitz constant of $u(q, a)$ with respect to $q$, we have

$$
D_{u, q} \leq \sup _{q \in[0.9,1]}|(\mathrm{d} / \mathrm{d} q) u(q, a)| \leq \sup _{q \in[0.9,1]} \lambda^{2} q / 2 \leq \lambda^{2} / 2
$$

For $(f, F)=(q, Q)$ or $(\varphi, M)$, we have

$$
|f-\mathbb{E}[F(x)]| \leq\left|f-f_{\star}\right|+D_{F, q}\left|q-q_{\star}\right|+D_{F, \varphi}\left|\varphi-\varphi_{\star}\right| .
$$

For $\lambda \geq 10$, we have $D_{Q, q} \vee D_{Q, \varphi} \vee D_{M, q} \vee D_{M, \varphi} \leq 1 / 2$, and hence

$$
\max \left\{\left|q-q_{\star}\right|,\left|\varphi-\varphi_{\star}\right|\right\} \leq L_{q}^{2} / 2
$$

implies (6.9).

Moreover, for $(f, F)=(q, Q),(\varphi, M)$, or $(a, A)$, we have

$$
\begin{aligned}
(f-\mathbb{E}[F(x)])^{2} & \geq\left(\left|f-f_{\star}\right|-D_{F, q}\left|q-q_{\star}\right|-D_{F, \varphi}\left|\varphi-\varphi_{\star}\right|\right)_{+}^{2} \\
& \geq\left(f-f_{\star}\right)^{2}-2\left|f-f_{\star}\right| \cdot\left(D_{F, q}\left|q-q_{\star}\right|+D_{F, \varphi}\left|\varphi-\varphi_{\star}\right|\right) \\
& \geq(1-\varepsilon)\left(f-f_{\star}\right)^{2}-(1 / \varepsilon)\left(D_{F, q}\left|q-q_{\star}\right|+D_{F, \varphi}\left|\varphi-\varphi_{\star}\right|\right)^{2} \\
& \geq(1-\varepsilon)\left(f-f_{\star}\right)^{2}-(2 / \varepsilon) D_{F, q}^{2}\left(q-q_{\star}\right)^{2}-(2 / \varepsilon) D_{F, \varphi}^{2}\left(\varphi-\varphi_{\star}\right)^{2},
\end{aligned}
$$

and

$$
\begin{aligned}
(u(q, a)-e-\mathbb{E}[U])^{2} & \\
\geq & \left(\left|\left(e-e_{\star}\right)-\left(a-a_{\star}\right) / 2\right|-\left(D_{U, q}+D_{u, q}\right)\left|q-q_{\star}\right|-D_{U, \varphi}\left|\varphi-\varphi_{\star}\right|\right)_{+}^{2} \\
\geq & (1-\varepsilon)\left[\left(e-e_{\star}\right)-\left(a-a_{\star}\right) / 2\right]^{2}-(2 / \varepsilon)\left(D_{U, q}+D_{u, q}\right)^{2}\left(q-q_{\star}\right)^{2} \\
& -(2 / \varepsilon) D_{U, \varphi}^{2}\left(\varphi-\varphi_{\star}\right)^{2} .
\end{aligned}
$$

Accordingly, for $\lambda_{0}$ sufficiently large, using equation (6.10), we obtain

$$
\begin{aligned}
& \inf _{\mu, \nu, \tau, \gamma} S_{-}(q, \varphi, a, e ; \mu, \nu, \tau, \gamma) \\
& \leq- \frac{1-\varepsilon}{2 \alpha L_{a}^{2} \lambda^{2}}\left(a-a_{\star}\right)^{2} \\
&- {\left[\frac{1-\varepsilon}{4 \kappa L_{q}^{2}}-\frac{2 D_{A, q}^{2}}{2 \varepsilon \alpha L_{a}^{2} \lambda^{2}}-\frac{2 D_{Q, q}^{2}}{4 \varepsilon \kappa L_{q}^{2}}-\frac{2 D_{M, q}^{2}}{4 \varepsilon \kappa L_{q}^{2}}-\frac{2\left(D_{U, q}+D_{u, q}\right)^{2}}{2 \varepsilon \kappa \lambda^{2}}\right]\left(q-q_{\star}\right)^{2} } \\
&- {\left[\frac{1-\varepsilon}{4 \kappa L_{q}^{2}}-\frac{2 D_{A, \varphi}^{2}}{2 \varepsilon \alpha L_{a}^{2} \lambda^{2}}-\frac{2 D_{Q, \varphi}^{2}}{4 \varepsilon \kappa L_{q}^{2}}-\frac{2 D_{M, \varphi}^{2}}{4 \varepsilon \kappa L_{q}^{2}}-\frac{2 D_{U, \varphi}^{2}}{2 \varepsilon \kappa \lambda^{2}}\right]\left(\varphi-\varphi_{\star}\right)^{2} } \\
&- \frac{1-\varepsilon}{2 \kappa \lambda^{2}}\left[\left(e-e_{\star}\right)-\left(a-a_{\star}\right) / 2\right]^{2},
\end{aligned}
$$

as long as $(q, \varphi)$ satisfies (6.11).

Step 5. Finish the proof. Let us apply

$$
S_{\star}(q, \varphi, a, e)=S_{+}(q, \varphi, a)+\inf _{\mu, \nu, \tau, \gamma} S_{-}(q, \varphi, a, e ; \mu, v, \tau, \gamma)
$$


and add the upper bounds from (6.2) and (6.12). As $\varepsilon=0.01, \alpha=1+\varepsilon=1.01, \kappa=(3+$ $3 \varepsilon) / \varepsilon=303$ and $L_{a} \leq 1.2$, the coefficient for the $\left(a-a_{\star}\right)^{2}$ term

$$
-\frac{1-\varepsilon}{2 \alpha L_{a}^{2} \lambda^{2}}+\frac{(1+\varepsilon)^{4}}{4 \lambda^{2}}
$$

is a negative function of $\lambda$. Now, take $L_{q}^{2}=2 c_{0} / \lambda^{2}$ for some small constant $c_{0}>0$. For $\lambda_{0}$ large enough, this implies $L_{q}^{2} \geq \exp \left\{-\lambda^{2} / 10\right\}$.

Note that $D_{Q, q}, D_{Q, \varphi}, D_{M, q}, D_{M, \varphi}$ are exponentially small in $\lambda$, while $D_{A, q}, D_{A, \varphi}, D_{U, q}$, $D_{u, q}, D_{U, \varphi} \leq 2 \lambda^{2}$. Hence, for $c_{0}$ small enough and $\lambda_{0}$ large enough, the coefficients for the $\left(q-q_{\star}\right)^{2}$ and $\left(\varphi-\varphi_{\star}\right)^{2}$ terms are also negative functions of $\lambda$; moreover, $\max \{\mid q-$ $\left.q_{\star}|,| \varphi-\varphi_{\star} \mid\right\} \leq c_{0} / \lambda^{2}$ implies (6.11). Finally, observe that, for any $c_{1}(\lambda), c_{2}(\lambda)>0$, there exists $c(\lambda)>0$ such that

$$
-c_{1}(\lambda)\left(a-a_{\star}\right)^{2}-c_{2}(\lambda)\left[\left(e-e_{\star}\right)-\left(a-a_{\star}\right) / 2\right]^{2} \leq-c(\lambda)\left(a-a_{\star}\right)^{2}-c(\lambda)\left(e-e_{\star}\right)^{2} .
$$

This concludes the proof of (5.4).

\section{APPENDIX A: PROOF OF PROPOSITION 3.1}

Fix $n$, and write as shorthand $\boldsymbol{g} \equiv \boldsymbol{g}_{n}$ and $\boldsymbol{H} \equiv \boldsymbol{H}_{n}$. For any measurable $T \subseteq(-1,1)^{n}$, define

$$
\operatorname{Crit}(T)=\sum_{\boldsymbol{m}: \boldsymbol{g}(\boldsymbol{m})=\mathbf{0}} \mathbf{1}\{\boldsymbol{m} \in T\} .
$$

We wish to apply the Kac-Rice formula [1], Theorem 11.2.1, for $\mathbb{E}[\operatorname{Crit}(T)]$. The statement of [1], Theorem 11.2.1, does not directly apply in our setting, as $\boldsymbol{g}(\boldsymbol{m})$ and $\boldsymbol{H}(\boldsymbol{m})=\nabla \boldsymbol{g}(\boldsymbol{m})$ do not admit a joint density on $\mathbb{R}^{n} \times \mathbb{R}^{n(n+1) / 2}$. (Conditional on $\boldsymbol{H}(\boldsymbol{m})$, the gradient $\boldsymbol{g}(\boldsymbol{m})$ is deterministic.) We will instead adapt the proof presented in [1] to handle this technicality. For simplicity, we prove only the upper bound which is all that we require for our application.

Let $\mathrm{B}(\delta)$ be the open ball of radius $\delta$ around $\mathbf{0}$. Proposition 3.1 is an immediate consequence of the following lemma.

LEMMA A.1. Let $p_{\boldsymbol{m}}$ be the Lebesgue density of $\boldsymbol{g}(\boldsymbol{m})$. Let $\delta \equiv \delta_{n}>0$. Then, for any Borel measurable set $T \subseteq V_{\delta} \equiv[-1+\delta, 1-\delta]^{n} \backslash \mathrm{B}(\delta)$,

$$
\mathbb{E}[\operatorname{Crit}(T)] \leq \int \mathbf{1}\{\boldsymbol{m} \in T\} \mathbb{E}[|\operatorname{det} \boldsymbol{H}(\boldsymbol{m})| \mid \boldsymbol{g}(\boldsymbol{m})=\mathbf{0}] p_{\boldsymbol{m}}(\mathbf{0}) \mathrm{d} \boldsymbol{m} .
$$

To prove this lemma, we apply the following result from [1]: Define the smoothed delta function

$$
\delta_{\varepsilon}(\boldsymbol{m})= \begin{cases}\operatorname{Vol}(\mathrm{B}(\varepsilon))^{-1} & \boldsymbol{m} \in \mathrm{B}(\varepsilon), \\ 0 & \boldsymbol{m} \notin \mathrm{B}(\varepsilon)\end{cases}
$$

which integrates to 1 over $\mathbb{R}^{n}$.

LEMMA A.2. Suppose $\mathrm{g}:(-1,1)^{n} \rightarrow \mathbb{R}^{n}$ is deterministic and continuously differentiable, and $T \subset(-1,1)^{n}$ is compact. Suppose, furthermore, that there are no points $\boldsymbol{m} \in T$ satisfying both $\boldsymbol{g}(\boldsymbol{m})=\mathbf{0}$ and det $\nabla \boldsymbol{g}(\boldsymbol{m})=\mathbf{0}$ and also no points $\boldsymbol{m} \in \partial T$ satisfying $\boldsymbol{g}(\boldsymbol{m})=\mathbf{0}$. Then,

$$
\operatorname{Crit}(T)=\lim _{\varepsilon \rightarrow 0} \int \mathbf{1}\{\boldsymbol{m} \in T\} \delta_{\varepsilon}(\boldsymbol{g}(\boldsymbol{m}))|\operatorname{det} \nabla \boldsymbol{g}(\boldsymbol{m})| \mathrm{d} \boldsymbol{m}
$$


ProOF. See [1], Theorem 11.2.3.

The below verifies that the conditions required for Lemma A.2 hold almost surely.

LEMMA A.3. Let $\boldsymbol{g} \equiv \boldsymbol{g}_{n}$ be defined by (3.2), and let $T \subset(-1,1)^{n}$ be compact with $\mathbf{0} \notin T$ and $\partial T$ having Lebesgue measure 0. Then, the conditions of Lemma A.2 hold with probability 1 .

ProOF. Let us first verify that, with probability 1 , no point $\boldsymbol{m} \in \partial T$ satisfies $\boldsymbol{g}(\boldsymbol{m})=$ 0. Fix $C_{0}>0$, and consider the event $\mathcal{E}=\left\{\|\boldsymbol{W}\|_{\text {op }}<C_{0}\right\}$. As $T$ is compact and does not contain $\mathbf{0}$, it belongs to $K_{2 \delta} \equiv[-1+2 \delta, 1-2 \delta]^{n} \backslash(-2 \delta, 2 \delta)^{n}$ for some $(n, T)$-dependent quantity $\delta>0$. Since $\partial T$ has Lebesgue outer measure 0 , for any $\varepsilon>0$ there exists a countable collection of balls $\left\{\mathrm{B}\left(\boldsymbol{m}_{i}, r_{i}\right): i \in \mathcal{I}\right\}$ such that

$$
\sum_{i \in \mathcal{I}} r_{i}^{n}<\varepsilon, \quad \partial T \subset \bigcup_{i \in \mathcal{I}} \mathrm{B}\left(\boldsymbol{m}_{i}, r_{i}\right),
$$

where each $\mathrm{B}\left(\boldsymbol{m}_{i}, r_{i}\right)$ is the open ball of radius $r_{i}$ around $\boldsymbol{m}_{i}$. Taking $\varepsilon<\delta^{n}$ so that $r_{i}<\delta$ for each $i$, we may assume without loss of generality that each center $\boldsymbol{m}_{i}$ belongs to $K_{\delta}$ (because otherwise the ball has empty intersection with $K_{2 \delta}$ and, therefore, with $T$ ). On the event $\mathcal{E}$, $\boldsymbol{g}(\boldsymbol{m})$ is $L$-Lipschitz over $K_{\delta}$ for some $\left(n, \delta, C_{0}\right)$-dependent quantity $L>0$. Hence,

$$
\mathbb{P}[\mathcal{E} \cap\{\text { there exists } \boldsymbol{m} \in \partial T: \boldsymbol{g}(\boldsymbol{m})=0\}] \leq \sum_{i \in \mathcal{I}} \mathbb{P}\left[\left\|\boldsymbol{g}\left(\boldsymbol{m}_{i}\right)\right\|_{2}<L r_{i}\right] .
$$

Observe that, for each fixed $\boldsymbol{m}_{i}$, the vector $\boldsymbol{g}\left(\boldsymbol{m}_{i}\right)$ has a multivariate normal distribution with covariance

$$
\beta^{2}\left(n^{-1}\left\|\boldsymbol{m}_{i}\right\|_{2}^{2} \mathbf{I}+n^{-1} \boldsymbol{m}_{i} \boldsymbol{m}_{i}^{\top}\right) \succeq \beta^{2} \delta^{2} \mathbf{I} .
$$

Then, the density of $\boldsymbol{g}\left(\boldsymbol{m}_{i}\right)$ is bounded as $\varphi_{i}(\boldsymbol{x}) \leq\left(2 \pi \beta^{2} \delta^{2}\right)^{-n / 2}$, and, for some $C=C(\beta)>$ 0 not depending on $\varepsilon, \delta$, we have

$$
\mathbb{P}\left[\left\|\boldsymbol{g}\left(\boldsymbol{m}_{i}\right)\right\|_{2}<L r_{i}\right] \leq\left(\frac{C L r_{i}}{\delta}\right)^{n}
$$

Hence,

$$
\mathbb{P}[\mathcal{E} \cap\{\text { there exists } \boldsymbol{m} \in \partial T: \boldsymbol{g}(\boldsymbol{m})=0\}] \leq \sum_{i \in \mathcal{I}}\left(\frac{C L r_{i}}{\delta}\right)^{n} \leq\left(\frac{C L}{\delta}\right)^{n} \varepsilon .
$$

As $\varepsilon>0$ is arbitrary, the above probability must be 0 . Then,

$$
\mathbb{P}[\text { there exists } \boldsymbol{m} \in \partial T: \boldsymbol{g}(\boldsymbol{m})=0] \leq 1-\mathbb{P}[\mathcal{E}] .
$$

Now, taking $C_{0} \rightarrow \infty$,

$$
\mathbb{P}[\text { there exists } \boldsymbol{m} \in \partial T: \boldsymbol{g}(\boldsymbol{m})=0]=0 .
$$

Next, let us verify that, with probability 1 , no point $\boldsymbol{m} \in T$ satisfies both $\boldsymbol{g}(\boldsymbol{m})=0$ and $\operatorname{det} \boldsymbol{H}(\boldsymbol{m})=0$. Define the set

$$
S=\left\{\boldsymbol{m} \in(-1,1)^{n}: \frac{m_{i}}{1-m_{i}^{2}}-\frac{2 \beta^{2}}{n}\|\boldsymbol{m}\|_{2}^{2} \cdot m_{i}-\operatorname{arctanh}\left(m_{i}\right)=0 \forall i \in[n]\right\},
$$

and suppose first that $T \cap S=\varnothing$. Note that, for any $r>0$, we may construct a maximal packing $\left\{\mathrm{B}\left(\boldsymbol{m}_{i}, r / 2\right): i \in \mathcal{I}\right\}$ where $\boldsymbol{m}_{i} \in T$ for each $i$. Namely, these balls do not intersect, and no additional ball $\mathrm{B}(\boldsymbol{m}, r / 2)$ with $\boldsymbol{m} \in T$ may be added to the packing. Then, $\left\{\mathrm{B}\left(\boldsymbol{m}_{i}, r\right)\right.$ : 
$i \in \mathcal{I}\}$ is a finite cover of $T$, and a volume argument shows $|\mathcal{I}| \leq C / r^{n}$ for an $n$-dependent constant $C>0$. As $T$ is compact and $S$ is closed, $T$ is included in the set

$$
U_{\delta}=\left\{\boldsymbol{m} \in[-1+\delta, 1-\delta]^{n}: \operatorname{dist}(\boldsymbol{m}, S) \geq \delta,\|\boldsymbol{m}\|_{2} \geq \delta\right\}
$$

for some $(n, T)$-dependent $\delta>0$. Fixing $C_{0}>0$ and defining the event $\mathcal{E}=\left\{\|\boldsymbol{W}\|_{\text {op }}<C_{0}\right\}$, we have on $\mathcal{E}$ that $\boldsymbol{g}(\cdot)$ and $\operatorname{det}(\boldsymbol{H}(\cdot))$ are both $L$-Lipschitz over $\boldsymbol{m} \in U_{\delta}$ for some $L=$ $L\left(n, \delta, C_{0}\right)>0$. Hence,

$$
\begin{aligned}
\mathbb{P} & {[\mathcal{E} \cap\{\text { there exists } \boldsymbol{m} \in T: \boldsymbol{g}(\boldsymbol{m})=0, \operatorname{det} \boldsymbol{H}(\boldsymbol{m})=0\}] } \\
& \leq \sum_{i \in \mathcal{I}} \mathbb{P}\left[\mathcal{E},\left\|\boldsymbol{g}\left(\boldsymbol{m}_{i}\right)\right\|_{2}<L r,\left|\operatorname{det} \boldsymbol{H}\left(\boldsymbol{m}_{i}\right)\right|<L r\right] .
\end{aligned}
$$

Consider a fixed index $i$, and let $\boldsymbol{v}$ and $\boldsymbol{A}$ be such that

$$
\boldsymbol{g}\left(\boldsymbol{m}_{i}\right)=-\beta\left(\boldsymbol{W} \boldsymbol{m}_{i}+\boldsymbol{v}\right), \quad \boldsymbol{H}\left(\boldsymbol{m}_{i}\right)=-\beta(\boldsymbol{W}+\boldsymbol{A})
$$

for $\boldsymbol{g}, \boldsymbol{H}$, as defined in (3.2) and (3.3). Since $\boldsymbol{m}_{i} \in U_{\delta}$, the conditions of Lemma A.4 below are satisfied for some quantities $c_{0} \equiv c_{0}(n, \delta, \lambda, \beta)>0$ and $C_{0} \equiv C_{0}(n, \delta, \lambda, \beta)>0$. Then, we obtain, for all $r$ sufficiently small and some $C>0$ independent of $r$,

$$
\mathbb{P}\left[\mathcal{E},\left\|\boldsymbol{g}\left(\boldsymbol{m}_{i}\right)\right\|_{2}<L r,\left|\operatorname{det} \boldsymbol{H}\left(\boldsymbol{m}_{i}\right)\right|<L r\right]<C r^{n+1 /(3 n)} .
$$

Applying this and $|\mathcal{I}| \leq C / r^{n}$ to the above and taking $r \rightarrow 0$ followed by $C_{0} \rightarrow \infty$, we obtain

$$
\mathbb{P}[\text { there exists } \boldsymbol{m} \in T: \boldsymbol{g}(\boldsymbol{m})=0, \operatorname{det} \boldsymbol{H}(\boldsymbol{m})=0]=0 .
$$

If $T \cap S \neq \varnothing$, this argument holds for the compact set $T \backslash\{\boldsymbol{m}: \operatorname{dist}(\boldsymbol{m}, S)<\delta\}$ and any $\delta>0$. Taking a union bound over a countable sequence $\delta \rightarrow 0$ and noting that $T \cap S$ is closed, we obtain

$$
\mathbb{P}[\text { there exists } \boldsymbol{m} \in T \backslash S: \boldsymbol{g}(\boldsymbol{m})=0, \operatorname{det} \boldsymbol{H}(\boldsymbol{m})=0]=0 .
$$

Finally, note that $S$ is the zero set of a nontrivial real analytic function, and thus $S$ has Lebesgue measure 0 [48]. The same argument as for $\partial T$ shows $\mathbb{P}[$ there exists $\boldsymbol{m} \in T \cap S$ : $\boldsymbol{g}(\boldsymbol{m})=0]=0$, and combining the above,

$$
\mathbb{P}[\text { there exists } \boldsymbol{m} \in T: \boldsymbol{g}(\boldsymbol{m})=0, \operatorname{det} \boldsymbol{H}(\boldsymbol{m})=0]=0 .
$$

LEMMA A.4. For any (n-dependent) quantities $c_{0}, C_{0}>0$, there exist $C \equiv C\left(n, c_{0}\right.$, $\left.C_{0}\right)>0$ and $\varepsilon_{0} \equiv \varepsilon_{0}\left(n, c_{0}, C_{0}\right)>0$ such that the following holds: Let $\boldsymbol{v} \in \mathbb{R}^{n}, \boldsymbol{m} \in(-1,1)^{n}$ and $\boldsymbol{A} \in \mathbb{R}^{n \times n}$ be any deterministic vectors/matrices such that $\boldsymbol{A}$ is symmetric, $\|\boldsymbol{A}\|_{\mathrm{op}}<C_{0}$, $\|\boldsymbol{A} \boldsymbol{m}-\boldsymbol{v}\|_{2}>c_{0}$ and $\|\boldsymbol{m}\|_{2}>c_{0}$. Let $\boldsymbol{W} \sim \operatorname{GOE}(n)$. Then, for all $\varepsilon \in\left(0, \varepsilon_{0}\right)$,

$$
\mathbb{P}\left[\|\boldsymbol{W} \boldsymbol{m}+\boldsymbol{v}\|_{2}<\varepsilon \text { and }|\operatorname{det}(\boldsymbol{W}+\boldsymbol{A})|<\varepsilon \text { and }\|\boldsymbol{W}\|_{\text {op }}<C_{0}\right]<C \varepsilon^{n+1 /(3 n)} \text {. }
$$

PROOF. Throughout the proof, $C$ and $c>0$ denote arbitrary $\left(n, c_{0}, C_{0}\right)$-dependent constants that may change from line to line.

Note that $\boldsymbol{W} \boldsymbol{m}+\boldsymbol{v}$ has a multivariate normal distribution with covariance

$$
n^{-1}\|\boldsymbol{m}\|_{2}^{2} \mathbf{I}+n^{-1} \boldsymbol{m} \boldsymbol{m}^{\top} \succeq n^{-1} c_{0}^{2} \mathbf{I} .
$$

Then, the density of $\boldsymbol{W} \boldsymbol{m}+\boldsymbol{v}$ is bounded by an $n$-dependent constant, so $\mathbb{P}\left[\|\boldsymbol{W} \boldsymbol{m}+\boldsymbol{v}\|_{2}<\right.$ $\varepsilon]<C \varepsilon^{n}$. Hence, it suffices to show

$$
\mathbb{P}\left[|\operatorname{det}(\boldsymbol{W}+\boldsymbol{A})|<\varepsilon,\|\boldsymbol{W}\|_{\text {op }}<C_{0} \mid \boldsymbol{W} \boldsymbol{m}=\boldsymbol{w}\right]<C \varepsilon^{1 /(3 n)}
$$

for any deterministic $\boldsymbol{w}$ satisfying $\|\boldsymbol{w}+\boldsymbol{v}\|_{2}<\varepsilon<\varepsilon_{0}$. 
For this, define a deterministic orthogonal matrix $\boldsymbol{O} \in \mathbb{R}^{n \times n}$ such that its first column is $\boldsymbol{m} /\|\boldsymbol{m}\|_{2}$, and the span of its first two columns contains $\boldsymbol{m}$ and $\boldsymbol{A} \boldsymbol{m}+\boldsymbol{w}$. Set

$$
\check{\boldsymbol{W}}=\boldsymbol{O}^{\top} \boldsymbol{W} \boldsymbol{O}, \quad \check{\boldsymbol{w}}=\boldsymbol{O}^{\top} \boldsymbol{w}, \quad \check{\boldsymbol{A}}=\boldsymbol{O}^{\top} \boldsymbol{A} \boldsymbol{O} .
$$

Then, rotating coordinates and denoting by $\boldsymbol{e}_{i}$ the $i$ th standard basis vector,

$$
\begin{aligned}
& \mathbb{P}\left[|\operatorname{det}(\boldsymbol{W}+\boldsymbol{A})|<\varepsilon,\|\boldsymbol{W}\|_{\text {op }}<C_{0} \mid \boldsymbol{W} \boldsymbol{m}=\boldsymbol{w}\right] \\
& =\mathbb{P}\left[|\operatorname{det}(\check{\boldsymbol{W}}+\check{\boldsymbol{A}})|<\varepsilon,\|\check{\boldsymbol{W}}\|_{\text {op }}<C_{0} \mid\|\boldsymbol{m}\|_{2} \check{\boldsymbol{W}} \boldsymbol{e}_{1}=\check{\boldsymbol{w}}\right] .
\end{aligned}
$$

Conditional on $\|\boldsymbol{m}\|_{2} \check{\boldsymbol{W}} \boldsymbol{e}_{1}=\check{\boldsymbol{w}}$, the first column (and also first row) of $\check{\boldsymbol{W}}+\check{\boldsymbol{A}}$ is deterministic and given by

$$
(\check{\boldsymbol{W}}+\check{\boldsymbol{A}}) \boldsymbol{e}_{1}=\frac{\check{\boldsymbol{w}}}{\|\boldsymbol{m}\|_{2}}+\check{\boldsymbol{A}} \boldsymbol{e}_{1}=\frac{1}{\|\boldsymbol{m}\|_{2}} \boldsymbol{O}^{\top}(\boldsymbol{w}+\boldsymbol{A m})=\alpha_{1} \boldsymbol{e}_{1}+\alpha_{2} \boldsymbol{e}_{2},
$$

where the last equality holds by construction of $\boldsymbol{O}$ for some scalars $\alpha_{1}, \alpha_{2}$ which satisfy $\alpha_{1}^{2}+$ $\alpha_{2}^{2}=\|\boldsymbol{A} \boldsymbol{m}+\boldsymbol{w}\|_{2}^{2} /\|\boldsymbol{m}\|_{2}^{2}$. Then, denoting by $\boldsymbol{H}^{(1)}=\check{\boldsymbol{W}}^{(1)}+\check{\boldsymbol{A}}^{(1)}$ and $\boldsymbol{H}^{(12)}=\check{\boldsymbol{W}}^{(12)}+\check{\boldsymbol{A}}^{(12)}$ the lower right $(n-1) \times(n-1)$ and $(n-2) \times(n-2)$ submatrices of $\boldsymbol{W}+\boldsymbol{A}$, and expanding the determinant along the first column,

$$
\operatorname{det}(\check{\boldsymbol{W}}+\check{\boldsymbol{A}})=\alpha_{1} \operatorname{det} \boldsymbol{H}^{(1)}-\alpha_{2}^{2} \operatorname{det} \boldsymbol{H}^{(12)} .
$$

For sufficiently small $\varepsilon_{0}$, the given conditions and $\|\boldsymbol{w}+\boldsymbol{v}\|_{2}<\varepsilon_{0}$ imply $\alpha_{1}^{2}+\alpha_{2}^{2}>c$. We consider two cases:

- Case 1: $\left|\alpha_{1}\right|<\varepsilon^{1 / 3}$. Then, $\alpha_{2}^{2}>c$ for a constant $c>0$. For $\|\boldsymbol{W}\|_{\text {op }} \leq C_{0}$ and $\|\boldsymbol{A}\|_{\text {op }} \leq C_{0}$, we have $\left\|\boldsymbol{H}^{(1)}\right\|_{\text {op }} \leq 2 C_{0}$ and hence $\left|\operatorname{det}\left(\boldsymbol{H}^{(1)}\right)\right| \leq\left(2 C_{0}\right)^{n-1}$. Combining with $\mid \operatorname{det}(\check{\boldsymbol{W}}+$ $\check{\boldsymbol{A}}) \mid<\varepsilon$, we have $\left|\operatorname{det} \boldsymbol{H}^{(12)}\right|<C \varepsilon^{1 / 3}$ for some constant $C>0$. Also, $\|\boldsymbol{W}\|_{\text {op }}<C_{0}$ and $\|\boldsymbol{A}\|_{\text {op }} \leq C_{0}$ imply $\left\|\boldsymbol{H}^{(12)}\right\|<2 C_{0}$. Hence,

$$
\begin{gathered}
\mathbb{P}\left[|\operatorname{det}(\check{\boldsymbol{W}}+\check{\boldsymbol{A}})|<\varepsilon,\|\check{\boldsymbol{W}}\|_{\text {op }}<C_{0} \mid\|\boldsymbol{m}\|_{2} \check{\boldsymbol{W}} \boldsymbol{e}_{1}=\check{\boldsymbol{w}}\right] \\
\leq \mathbb{P}\left[\left|\operatorname{det} \boldsymbol{H}^{(12)}\right|<C \varepsilon^{1 / 3},\left\|\boldsymbol{H}^{(12)}\right\|_{\text {op }}<2 C_{0}\right]
\end{gathered}
$$

for a constant $C>0$ and sufficiently small $\varepsilon$. Writing the spectral decomposition $\boldsymbol{H}^{(12)}=$ $\boldsymbol{U} \boldsymbol{\Lambda} \boldsymbol{U}^{\top}$ where $\Lambda=\operatorname{diag}\left(\lambda_{1}, \ldots, \lambda_{n-2}\right)$ and applying the change of variables $\mathrm{d} \boldsymbol{H}^{(12)}=$ $\left(1 / Z^{\prime}\right) \prod_{i<j}\left|\lambda_{i}-\lambda_{j}\right| \mathrm{d} \Lambda \mathrm{d} \boldsymbol{U}[3]$, the joint density of ordered eigenvalues $\lambda_{1}, \ldots, \lambda_{n-2}$ of $\boldsymbol{H}^{(12)}$ is given by

$$
\begin{aligned}
& (1 / Z) \mathbf{1}\left\{\lambda_{1} \leq \cdots \leq \lambda_{n-2}\right\} \prod_{i<j}\left|\lambda_{j}-\lambda_{i}\right| \\
& \cdot \int \exp \left(-\frac{n}{4} \operatorname{Tr}\left[\left(\boldsymbol{U} \boldsymbol{\Lambda} \boldsymbol{U}^{\top}-\check{\boldsymbol{A}}^{(12)}\right)^{2}\right]\right) \mathrm{d} \boldsymbol{U},
\end{aligned}
$$

where the integral is over the orthogonal group of dimension $n-2$. This density is bounded by an $n$-dependent constant over the set

$$
\left\{\lambda_{1} \leq \cdots \leq \lambda_{n-2}: \prod_{i}\left|\lambda_{i}\right|<C \varepsilon^{1 / 3}, \max _{i}\left|\lambda_{i}\right|<2 C_{0}\right\},
$$

and the Lebesgue volume of this set is bounded by $C \varepsilon^{1 /(3 n)}$ since at least one coordinate $\left|\lambda_{i}\right|$ is less than $C \varepsilon^{1 /(3 n)}$. Then, the right side of (A.2) is at most $C \varepsilon^{1 /(3 n)}$ for a constant $C>0$. 
- Case 2: $\left|\alpha_{1}\right| \geq \varepsilon^{1 / 3}$. Considering separately the events $\left|\operatorname{det} \boldsymbol{H}^{(12)}\right|<\varepsilon^{1 / 3}$ and $\left|\operatorname{det} \boldsymbol{H}^{(12)}\right| \geq$ $\varepsilon^{1 / 3}$ and handling the first event by the argument of Case 1 above, we obtain

$$
\begin{aligned}
& \mathbb{P}\left[|\operatorname{det}(\check{\boldsymbol{W}}+\check{\boldsymbol{A}})|<\varepsilon,\|\check{\boldsymbol{W}}\|_{\mathrm{op}}<C_{0} \mid\|\boldsymbol{m}\|_{2} \check{\boldsymbol{W}} \boldsymbol{e}_{1}=\check{\boldsymbol{w}}\right] \\
& \quad \leq C \varepsilon^{1 /(3 n)}+\mathbb{P}\left[|\operatorname{det}(\check{\boldsymbol{W}}+\check{\boldsymbol{A}})|<\varepsilon,\left|\operatorname{det} \boldsymbol{H}^{(12)}\right| \geq \varepsilon^{1 / 3} \mid\|\boldsymbol{m}\|_{2} \check{\boldsymbol{W}} \boldsymbol{e}_{1}=\check{\boldsymbol{w}}\right] .
\end{aligned}
$$

For the probability on the right side, let us further condition on all entries of $\check{W}$ except $\check{W}_{22}$. We have

$$
\operatorname{det}(\check{\boldsymbol{W}}+\check{\boldsymbol{A}})=\alpha_{1} \check{W}_{22} \operatorname{det} \boldsymbol{H}^{(12)}+\text { const, }
$$

where const denotes a quantity that does not depend on $\check{W}_{22}$. Then, when $\left|\alpha_{1}\right| \geq \varepsilon^{1 / 3}$ and $\left|\operatorname{det} \boldsymbol{H}^{(12)}\right| \geq \varepsilon^{1 / 3}$, the quantity $\operatorname{det}(\check{\boldsymbol{W}}+\check{\boldsymbol{A}}) / \varepsilon$ is conditionally normally distributed with variance at least $2 \varepsilon^{-2 / 3} / n$. This normal distribution has density upper bounded by $C \varepsilon^{1 / 3}$, so

$$
\mathbb{P}\left[|\operatorname{det}(\check{\boldsymbol{W}}+\check{\boldsymbol{A}})|<\varepsilon,\left|\operatorname{det} \boldsymbol{H}^{(12)}\right| \geq \varepsilon^{1 / 3} \mid\|\boldsymbol{m}\|_{2} \check{\boldsymbol{W}} \boldsymbol{e}_{1}=\check{\boldsymbol{w}}\right] \leq C \varepsilon^{1 / 3} .
$$

Combining these cases yields (A.1), as desired.

Proof OF Lemma A.1. First, consider $T \subseteq V_{\delta} \equiv[-1+\delta, 1-\delta]^{n} \backslash \mathrm{B}(\delta)$ to be a closed hyperrectangle. By Lemma A.2, Lemma A.3, Fatou's Lemma and Fubini's Theorem,

$$
\mathbb{E}[\operatorname{Crit}(T)] \leq \liminf _{\varepsilon \rightarrow 0} \int \mathbf{1}\{\boldsymbol{m} \in T\} \mathbb{E}\left[\delta_{\varepsilon}(\boldsymbol{g}(\boldsymbol{m}))|\operatorname{det} \boldsymbol{H}(\boldsymbol{m})|\right] \mathrm{d} \boldsymbol{m} .
$$

Denoting by $p_{\boldsymbol{m}}$ the density of $\boldsymbol{g}(\boldsymbol{m})$, we have

$$
\begin{aligned}
\mathbb{E} & {\left[\delta_{\varepsilon}(\boldsymbol{g}(\boldsymbol{m}))|\operatorname{det} \boldsymbol{H}(\boldsymbol{m})|\right] } \\
& =\operatorname{Vol}(\mathrm{B}(\varepsilon))^{-1} \int_{\boldsymbol{u} \in \mathrm{B}(\varepsilon)} \mathbb{E}[|\operatorname{det} \boldsymbol{H}(\boldsymbol{m})| \mid \boldsymbol{g}(\boldsymbol{m})=\boldsymbol{u}] p_{\boldsymbol{m}}(\boldsymbol{u}) \mathrm{d} \boldsymbol{u} .
\end{aligned}
$$

Define

$$
D(\boldsymbol{u}, \boldsymbol{m}) \equiv \mathbb{E}[\mid \operatorname{det} \boldsymbol{H}(\boldsymbol{m}) \| \boldsymbol{g}(\boldsymbol{m})=\boldsymbol{u}] p_{\boldsymbol{m}}(\boldsymbol{u}) .
$$

For any fixed $\boldsymbol{m} \in V_{\delta}$, the vector $\boldsymbol{g}(\boldsymbol{m})$ is a Gaussian random vector, and $\mathbb{E}[\boldsymbol{g}(\boldsymbol{m})]$ and $\mathbb{E}\left[\boldsymbol{g}(\boldsymbol{m}) \boldsymbol{g}(\boldsymbol{m})^{\top}\right]$ are continuous functions in $\boldsymbol{m}$. Hence, the density $p_{\boldsymbol{m}}(\boldsymbol{u})$ is a continuous function of $(\boldsymbol{u}, \boldsymbol{m}) \in \overline{\mathrm{B}(\varepsilon)} \times T$. Moreover, by Lemma 4.1 we have $[\boldsymbol{H}(\boldsymbol{m}) \mid \boldsymbol{g}(\boldsymbol{m})=$ $\boldsymbol{u}] \stackrel{d}{=}-\beta \mathrm{P}_{\boldsymbol{m}}^{\perp} \boldsymbol{W} \mathrm{P}_{\boldsymbol{m}}^{\perp}+A(\boldsymbol{u}, \boldsymbol{m})$, where $\boldsymbol{W} \sim \mathrm{GOE}(n), \mathrm{P}_{\boldsymbol{m}}^{\perp}$ is the projection orthogonal to $\boldsymbol{m}$, and $A(\boldsymbol{u}, \boldsymbol{m})$ is continuous in $(\boldsymbol{u}, \boldsymbol{m}) \in \overline{\mathrm{B}(\varepsilon)} \times T$. Therefore, $D(\boldsymbol{u}, \boldsymbol{m})$ is continuous in $(\boldsymbol{u}, \boldsymbol{m}) \in \overline{\mathrm{B}(\varepsilon)} \times T$, and hence it is bounded on $\overline{\mathrm{B}(\varepsilon)} \times T$. The bounded convergence theorem and the continuity of $D(\boldsymbol{u}, \boldsymbol{m})$ yield

$$
\begin{aligned}
\mathbb{E}[\operatorname{Crit}(T)] & \leq \int \mathbf{1}\{\boldsymbol{m} \in T\} \liminf _{\varepsilon \rightarrow 0} \mathbb{E}\left[\delta_{\varepsilon}(\boldsymbol{g}(\boldsymbol{m}))|\operatorname{det} \boldsymbol{H}(\boldsymbol{m})|\right] \mathrm{d} \boldsymbol{m} \\
& =\int \mathbf{1}\{\boldsymbol{m} \in T\} D(\mathbf{0}, \boldsymbol{m}) \mathrm{d} \boldsymbol{m} .
\end{aligned}
$$

Finally, we generalize the above inequality to a general Lebesgue measurable set $E \subseteq V_{\delta}$. This follows by standard measure theory machinery, and we sketch the proof in the following.

For any Lebesgue measurable set $E \subseteq V_{\delta}$, define $\nu_{0}(E)=\mathbb{E}[\operatorname{Crit}(E)]$ and $v(E)=\int \mathbf{1}\{\boldsymbol{m} \in$ $E\} D(\mathbf{0}, \boldsymbol{m}) \mathrm{d} \boldsymbol{m}$. Define

$$
\mathcal{T} \equiv\left\{T \subseteq V_{\delta}: T \text { is closed hyperrectangle }\right\} .
$$


Let $v_{\star}$ be the outer measure generated by the set function $v: \mathcal{T} \rightarrow \mathbb{R}$, that is, for any $E \subset V_{\delta}$,

$$
v_{\star}(E) \equiv \inf \left\{\sum_{i=1}^{\infty} v\left(T_{i}\right): E \subseteq \bigcup_{i} T_{i}, T_{i} \in \mathcal{T}\right\} .
$$

Note that we have shown $v_{0}(T) \leq v(T)$ for any closed hyperrectangle $T \in \mathcal{T}$. Then, for any Lebesgue measurable set $E \subseteq V_{\delta}$,

$$
\begin{aligned}
v_{0}(E) & \stackrel{(i)}{\leq} \inf \left\{\sum_{i=1}^{\infty} v_{0}\left(T_{i}\right): E \subseteq \bigcup_{i} T_{i}, T_{i} \in \mathcal{T}\right\} \\
& \stackrel{(i i))}{\leq} \inf \left\{\sum_{i=1}^{\infty} v\left(T_{i}\right): E \subseteq \bigcup_{i} T_{i}, T_{i} \in \mathcal{T}\right\} \stackrel{(i i i)}{=} v_{\star}(E) .
\end{aligned}
$$

The inequality $(i)$ is given by the definition $v_{0}(E)=\mathbb{E}[\operatorname{Crit}(E)]$, the nonnegativity and additivity of Crit(.) and the linearity of expectation. The inequality $(i i)$ is given by $v_{0}\left(T_{i}\right) \leq v\left(T_{i}\right)$ for each $T_{i} \in \mathcal{T}$. The equality ( $\left.i i i\right)$ is given by the definition $v_{\star}$.

Since $D(\mathbf{0}, \boldsymbol{m})$ is bounded over $\boldsymbol{m} \in V_{\delta}$, we have that $v$ is absolutely continuous with respect to Lebesgue measure and $v_{\star}(E)=v(E)$ for any Lebesgue measurable set $E \subseteq V_{\delta}$ by a standard argument. This concludes the proof.

\section{APPENDIX B: PROOF OF PROPOSITION 3.5}

The proof applies the following version of Varadhan lemma's upper bound which is a small extension of [31], Lemma 4.3.6:

LEMMA B.1. Let $\left\{Z_{n}\right\}_{n=1}^{\infty}$ be random variables taking values in a regular topological space $\mathcal{X}$. Let $I: \mathcal{X} \rightarrow[0, \infty]$ be a lower semicontinuous rate function with compact level sets $\Psi(\alpha) \equiv\{x: I(x) \leq \alpha\}$. For all closed sets $A \subseteq \mathcal{X}$, suppose $Z_{n}$ satisfies the large deviations upper bound

$$
\limsup _{n \rightarrow \infty} \frac{1}{n} \log \mathbb{P}\left[Z_{n} \in A\right] \leq-\inf _{x \in A} I(x) .
$$

Consider an upper semicontinuous function $\phi: \mathcal{X} \rightarrow[-\infty, \infty)$ and a Borel set $A \subseteq \mathcal{X}$ such that $A \cap\{x: \phi(x) \geq-\alpha\}$ is closed for each $\alpha<\infty$, and

$$
C(\gamma) \equiv \limsup _{n \rightarrow \infty} \frac{1}{n} \log \mathbb{E}\left[e^{\gamma n \phi\left(Z_{n}\right)} \mathbf{1}\left\{Z_{n} \in A\right\}\right]<\infty
$$

for some $\gamma>1$. Then,

$$
\limsup _{n \rightarrow \infty} \frac{1}{n} \log \mathbb{E}\left[e^{n \phi\left(Z_{n}\right)} \mathbf{1}\left\{Z_{n} \in A\right\}\right] \leq \sup _{x \in A}(\phi(x)-I(x)) .
$$

PROOF. The proof follows [31], Lemmas 4.3.6 and 4.3.8, with minor modifications. Fixing $\alpha<\infty$ and $\delta>0$, by compactness and semicontinuity we may take a finite open cover $\left\{A_{x_{1}}, \ldots, A_{x_{N}}\right\}$ of $A \cap\{x: \phi(x) \geq-\alpha\} \cap\{x: I(x) \leq \alpha\}$ such that

$$
\inf _{y \in \overline{A_{x_{i}}}} I(y) \geq I\left(x_{i}\right)-\delta, \quad \sup _{y \in \overline{A_{x_{i}}}} \phi(y) \leq \phi\left(x_{i}\right)+\delta .
$$

Then,

$$
\limsup _{n \rightarrow \infty} \frac{1}{n} \log \mathbb{E}\left[e^{n \phi\left(Z_{n}\right)} \mathbf{1}\left\{Z_{n} \in A\right\}\right] \leq \max \left(E_{1}, \ldots, E_{N}, R_{1}, R_{2}\right)
$$


where

$$
\begin{aligned}
& E_{i}=\limsup _{n \rightarrow \infty} \frac{1}{n} \log \mathbb{E}\left[e^{n \phi\left(Z_{n}\right)} \mathbf{1}\left\{Z_{n} \in \overline{A_{x_{i}}}\right\}\right] \\
& R_{1}=\limsup _{n \rightarrow \infty} \frac{1}{n} \log \mathbb{E}\left[e^{n \phi\left(Z_{n}\right)} \mathbf{1}\left\{Z_{n} \in\{x: \phi(x)<-\alpha\}\right\}\right] \\
& R_{2}=\limsup _{n \rightarrow \infty} \frac{1}{n} \log \mathbb{E}\left[e^{n \phi\left(Z_{n}\right)} \mathbf{1}\left\{Z_{n} \in A \cap\{x: \phi(x) \geq-\alpha\} \cap A_{x_{1}}^{c} \cap \cdots \cap A_{x_{N}}^{c}\right\}\right] .
\end{aligned}
$$

Together, (B.1) and (B.3) imply $E_{i} \leq \phi\left(x_{i}\right)-I\left(x_{i}\right)+2 \delta$. Also, by definition, $R_{1} \leq-\alpha$. For $R_{2}$, denote $B=A \cap\{x: \phi(x) \geq-\alpha\} \cap A_{x_{1}}^{c} \cap \cdots \cap A_{x_{N}}^{c}$. Note that $B$ is closed and $I(x)>\alpha$ on $B$. Then, by Holder's inequality and (B.1) and (B.2),

$$
\begin{aligned}
R_{2} & \leq \limsup _{n \rightarrow \infty} \frac{1}{n} \log \left(\mathbb{E}\left[e^{\gamma n \phi\left(Z_{n}\right)} \mathbf{1}\left\{Z_{n} \in A\right\}\right]^{1 / \gamma} \mathbb{P}\left[Z_{n} \in B\right]^{1-1 / \gamma}\right) \\
& \leq \frac{1}{\gamma} C(\gamma)+\left(1-\frac{1}{\gamma}\right) \alpha .
\end{aligned}
$$

The result follows from combining these bounds and then taking $\alpha \rightarrow \infty$ and $\delta \rightarrow 0$.

We now apply this to our setting. We equip $\mathcal{P}$ with the topology of weak convergence and the corresponding Borel $\sigma$-algebra. The following lemmas verify the conditions needed in Lemma B.1:

\section{LEMMA B.2.}

(a) For any constants $\eta>0$ and $\beta>0$, there exists a constant $C \equiv C(\beta, \eta)<\infty$ such that $J(\rho, y) \leq C+y^{2} /\left(4 \beta^{2}\right)$ for all $\rho \in \mathcal{P}$ with $Q(\rho) \geq \eta$.

(b) $J:\{\rho \in \mathcal{P}: Q(\rho)>0\} \times \mathbb{R} \rightarrow[-\infty, \infty)$ is upper semicontinuous.

ProOF. For $(a)$ we apply the inequality $(c+1) a^{2}+\left(c^{-1}+1\right) b^{2} \geq(a+b)^{2}$ with $c=2$, $a=\operatorname{arctanh}(x)-\beta \lambda \varphi+\beta^{2}(1-q) x-y x$ and $b=y x$. Recalling $g(x ; \varphi, q, y)$ from equation (3.6), this yields

$$
\log g(x ; \varphi, q, y) \leq \log \frac{1}{1-x^{2}}-\frac{\left(\operatorname{arctanh}(x)-\beta \lambda \varphi+\beta^{2}(1-q) x\right)^{2}}{6 \beta^{2} q}+\frac{(y x)^{2}}{4 \beta^{2} q} .
$$

By the boundedness of $M(\rho)$ and $Q(\rho)$ and the comparison of $\log 1 /\left(1-x^{2}\right)$ and $\operatorname{arctanh}(x)^{2}$ as $x \rightarrow \pm 1$, we see that, for $\varphi=M(\rho)$ and $q=Q(\rho)$, the first two terms above are together upper-bounded by an $\eta$-dependent constant. Then,

$$
J(\rho, y) \leq C+\int \frac{(y x)^{2}}{4 \beta^{2} Q(\rho)} \rho(\mathrm{d} x)=C+\frac{y^{2}}{4 \beta^{2}}
$$

for a constant $C \equiv C(\beta, \eta)<\infty$.

For $(b)$, fix $y, y_{1}, y_{2}, \ldots \in \mathbb{R}$ such that $y_{i} \rightarrow y$ and $\rho, \rho_{1}, \rho_{2}, \ldots \in \mathcal{P}$ such that $\rho_{i} \rightarrow \rho$ weakly, $Q(\rho)>0$, and $Q\left(\rho_{i}\right)>0$ for all $i$. Then, $Q\left(\rho_{i}\right) \rightarrow Q(\rho)$, so there is a lower bound $\eta>0$ on all $Q\left(\rho_{i}\right)$, as well as a finite upper bound on all $y_{i}^{2}$. Fix a constant $\alpha \in \mathbb{R}$, and define

$$
\begin{aligned}
f_{i, \alpha}(x) & =\max \left(\alpha, \log g\left(x ; M\left(\rho_{i}\right), Q\left(\rho_{i}\right), y_{i}\right)\right), \\
f_{\alpha}(x) & =\max (\alpha, \log g(x ; M(\rho), Q(\rho), y)) .
\end{aligned}
$$

Then, $f_{i, \alpha}$ and $f_{\alpha}$ are uniformly bounded above and below over all $i$. Furthermore, there is a value $\delta>0$ such that $f_{i, \alpha}(x)=f_{\alpha}(x)=\alpha$ for all $x<-1+\delta$ and $x>1-\delta$ and all $i$. As 
$f_{i, \alpha}(x) \rightarrow f_{\alpha}(x)$ uniformly over $x \in[-1+\delta, 1-\delta]$, this implies $f_{i, \alpha}(x) \rightarrow f_{\alpha}(x)$ uniformly over $x \in(-1,1)$. Then,

$$
\begin{aligned}
& \left|\int f_{i, \alpha}(x) \rho_{i}(\mathrm{~d} x)-\int f_{\alpha}(x) \rho(\mathrm{d} x)\right| \\
& \quad \leq \int\left|f_{i, \alpha}(x)-f_{\alpha}(x)\right| \rho_{i}(\mathrm{~d} x)+\left|\int f_{\alpha}(x)\left(\rho_{i}-\rho\right)(\mathrm{d} x)\right| \rightarrow 0 .
\end{aligned}
$$

Hence,

$$
\begin{aligned}
\limsup _{i} J\left(\rho_{i}, y_{i}\right) & \leq \lim _{i}\left(\frac{\beta^{2}\left(1-Q\left(\rho_{i}\right)\right)^{2}}{2}-\frac{1}{2} \log \left(2 \pi \beta^{2} Q\left(\rho_{i}\right)\right)+\int f_{i, \alpha}(x) \rho_{i}(\mathrm{~d} x)\right) \\
& =\frac{\beta^{2}(1-Q(\rho))^{2}}{2}-\frac{1}{2} \log \left(2 \pi \beta^{2} Q(\rho)\right)+\int f_{\alpha}(x) \rho(\mathrm{d} x) .
\end{aligned}
$$

The left side is independent of $\alpha$. As $x \mapsto g(x ; M(\rho), Q(\rho), y)$ is bounded above, the monotone convergence theorem yields, for the right side,

$$
\lim _{\alpha \rightarrow-\infty} \int f_{\alpha}(x) \rho(\mathrm{d} x)=\int \log g(x ; M(\rho), Q(\rho), y) \rho(\mathrm{d} x) .
$$

Then, $\lim \sup _{i} J\left(\rho_{i}, y_{i}\right) \leq J(\rho, y)$, so $J$ is upper semicontinuous.

LemmA B.3. Fix any $\alpha \in(0, \infty)$, and suppose $\rho, \rho_{1}, \rho_{2}, \ldots \in \mathcal{P}$ are such that $\rho_{i} \rightarrow \rho$ weakly and

$$
\int_{-1}^{1} \operatorname{arctanh}(x)^{2} \rho(\mathrm{d} x) \leq \alpha, \quad \int_{-1}^{1} \operatorname{arctanh}(x)^{2} \rho_{i}(\mathrm{~d} x) \leq \alpha
$$

for all $i$. Then, $A\left(\rho_{i}\right) \rightarrow A(\rho)$ and $E\left(\rho_{i}\right) \rightarrow E(\rho)$.

PROOF. As $x^{2}$ and $h(x)$ are continuous and bounded over $(-1,1)$, it suffices to show

$$
\int x \operatorname{arctanh}(x) \rho_{i}(\mathrm{~d} x) \rightarrow \int x \operatorname{arctanh}(x) \rho(\mathrm{d} x) .
$$

For any $\delta \in(0,1)$ such that $-1+\delta$ and $1-\delta$ are continuity points of $\rho$, we have by weak convergence

$$
\int_{[-1+\delta, 1-\delta]} x \operatorname{arctanh}(x) \rho_{i}(\mathrm{~d} x) \rightarrow \int_{[-1+\delta, 1-\delta]} x \operatorname{arctanh}(x) \rho(\mathrm{d} x) .
$$

Denoting $I_{\delta}=(-1,-1+\delta) \cup(1-\delta, 1)$, by Cauchy-Schwarz

$$
\left(\int_{I_{\delta}} x \operatorname{arctanh}(x) \rho(\mathrm{d} x)\right)^{2} \leq \int_{I_{\delta}} x^{2} \rho(\mathrm{d} x) \cdot \int_{I_{\delta}} \operatorname{arctanh}(x)^{2} \rho(\mathrm{d} x) \leq \alpha \int_{I_{\delta}} x^{2} \rho(\mathrm{d} x),
$$

and similarly for $\rho_{i}$. As $\int_{I_{\delta}} x^{2} \rho_{i}(\mathrm{~d} x) \rightarrow \int_{I_{\delta}} x^{2} \rho(\mathrm{d} x)$ also by weak convergence, this yields

$$
\underset{i}{\limsup }\left|\int x \operatorname{arctanh}(x) \rho_{i}(\mathrm{~d} x)-\int x \operatorname{arctanh}(x) \rho(\mathrm{d} x)\right| \leq 2 \alpha \int_{I_{\delta}} x^{2} \rho(\mathrm{d} x) .
$$

Taking $\delta \rightarrow 0$ yields the claim.

LEMMA B.4. For each $\alpha<\infty$, the set

$$
\Xi(\alpha) \equiv\{(\rho, y) \in \mathcal{P} \times \mathbb{R}:(Q(\rho), M(\rho), A(\rho), E(\rho)) \in U, J(\rho, y) \geq-\alpha\}
$$

is closed. 
Proof. Suppose $(\rho, y),\left(\rho_{1}, y_{1}\right),\left(\rho_{2}, y_{2}\right), \ldots$ are such that $\left(\rho_{i}, y_{i}\right) \rightarrow(\rho, y)$ and $\left(\rho_{i}, y_{i}\right) \in \Xi(\alpha)$ for all $i$. Then, $M\left(\rho_{i}\right) \rightarrow M(\rho)$ and $Q\left(\rho_{i}\right) \rightarrow Q(\rho)$ by weak convergence. In particular, $Q(\rho) \geq \eta$, so $J$ is well defined at $\rho$ and $J(\rho, y) \geq \lim \sup _{i} J\left(\rho_{i}, y_{i}\right) \geq-\alpha$ by the upper semicontinuity established in Lemma B.2(b). Applying $(a-b)^{2} \geq\left(a^{2} / 2\right)-b^{2}$ and $\log 1 /\left(1-x^{2}\right) \leq \operatorname{arctanh}(x)^{2} /\left(8 \beta^{2}\right)+c$ for a constant $c \equiv c(\beta)<\infty$, we have

$$
\log g(x ; \varphi, q, y) \leq c+\operatorname{arctanh}(x)^{2}\left(\frac{1}{8 \beta^{2}}-\frac{1}{4 \beta^{2} q}\right)+\frac{\left(\beta \lambda \varphi-\beta^{2}(1-q) x+y x\right)^{2}}{2 \beta^{2} q} .
$$

Since $y_{i} \rightarrow y<\infty$, the above bound and the conditions $J\left(\rho_{i}, y_{i}\right) \geq-\alpha, J(\rho, y) \geq-\alpha$, $Q\left(\rho_{i}\right) \in[\eta, 1]$, and $Q(\rho) \in[\eta, 1]$ imply $\int_{-1}^{1} \operatorname{arctanh}(x)^{2} \rho_{i}(\mathrm{~d} x) \leq \kappa$ and $\int_{-1}^{1} \operatorname{arctanh}(x)^{2} \times$ $\rho(\mathrm{d} x) \leq \kappa$ for all $i$ and some $\kappa<\infty$. Then, Lemma B.3 implies $A\left(\rho_{i}\right) \rightarrow A(\rho)$ and $E\left(\rho_{i}\right) \rightarrow$ $E(\rho)$. As $U$ is closed, this implies $(Q(\rho), M(\rho), A(\rho), E(\rho)) \in U$, so $\Xi(\alpha)$ is closed as desired.

ProOF OF PROPOSITION 3.5. Let $\rho_{n}$ denote the empirical measure of $m_{1}, \ldots, m_{n} \stackrel{i i d}{\sim} \pi_{0}$, where $\pi_{0}$ is the uniform distribution on $[-1,1]$, and let $Y_{n} \sim \mathcal{N}\left(0, \beta^{2} / n\right)$ be independent of $\rho_{n}$. Then,

$$
\begin{aligned}
T\left(U, V_{n}\right)= & 2^{n} \cdot \mathbb{E}\left[\exp \left(n J\left(\rho_{n}, Y_{n}\right)+C_{0} n^{\max (0.9, b)}\right)\right. \\
& \left.\times \mathbb{1}\left\{\left(Q\left(\rho_{n}\right), M\left(\rho_{n}\right), A\left(\rho_{n}\right), E\left(\rho_{n}\right)\right) \in U\right\}\right] .
\end{aligned}
$$

We apply Lemma B.1 with $\mathcal{P} \times \mathbb{R}$ as $\mathcal{X}, J(\rho, y)$ as $\phi(x)$, and $\{(\rho, y) \in \mathcal{P} \times \mathbb{R}$ : $(Q(\rho), M(\rho), A(\rho), E(\rho)) \in U\}$ as $A$. By Sanov's theorem, $\rho_{n}$ satisfies a large deviation principle (LDP) with rate $n$ and rate function $\rho \mapsto H\left(\rho \mid \pi_{0}\right)$. Then, combining with the form of the normal density for $Y_{n},\left(\rho_{n}, Y_{n}\right)$ satisfies the LDP with rate function $I(\rho, y)=$ $H\left(\rho \mid \pi_{0}\right)+y^{2} /\left(2 \beta^{2}\right)$. This rate function is lower semicontinuous with compact level sets. Lemmas B.2(b) and B.4 verify the semicontinuity and closedness conditions required for $\phi(x)$ and $A$ in Lemma B.1. Note further that by Lemma B.2(a), for any $\gamma \in(1,2)$ and some $C \equiv C(\beta, \eta)<\infty$

$$
\begin{gathered}
\limsup _{n \rightarrow \infty} \frac{1}{n} \log \mathbb{E}\left[e^{\gamma n J\left(\rho_{n}, Y_{n}\right)} \mathbf{1}\left\{Q\left(\rho_{n}\right) \geq \eta\right\}\right. \\
\leq \limsup _{n \rightarrow \infty} \frac{1}{n} \log \mathbb{E}\left[e^{\gamma n\left(C+Y_{n}^{2} /\left(4 \beta^{2}\right)\right)}\right]=C .
\end{gathered}
$$

This verifies (B.2), so the result follows by Lemma B.1.

\section{APPENDIX C: PROOF OF PROPOSITION 5.3}

LEMMA C.1. Fix any $\alpha \in(1 / 2,1)$. Then, there exist constants $\lambda_{0}, n_{0}$ and $c_{0}>0$ so that, for all $\lambda \geq \lambda_{0}$ and $n \geq n_{0}$ with probability at least $1-e^{-c_{0} n}$, every $\boldsymbol{m} \in(-1,1)^{n}$ with $\mathcal{F}(\boldsymbol{m}) \leq-\alpha \lambda^{2} / 2$ satisfies

$$
\begin{aligned}
Q(\boldsymbol{m}) & \geq\left(2 \alpha-1-6 / \lambda-4 / \lambda^{2}\right)^{1 / 2}, \\
|M(\boldsymbol{m})| & \geq\left(2 \alpha-1-6 / \lambda-4 / \lambda^{2}\right)^{1 / 4} .
\end{aligned}
$$

ProOF. Note that we have

$$
\begin{aligned}
\mathcal{F}(\boldsymbol{m}) & \geq-1-\lambda /(2 n) \cdot\langle\boldsymbol{m}, \boldsymbol{W} \boldsymbol{m}\rangle-\left(\lambda^{2} / 2\right) M(\boldsymbol{m})^{2}-\left(\lambda^{2} / 4\right)(1-Q(\boldsymbol{m}))^{2} \\
& \geq-1-\lambda\|\boldsymbol{W}\|_{\mathrm{op}} / 2-\left(\lambda^{2} / 2\right) M(\boldsymbol{m})^{2}-\left(\lambda^{2} / 4\right)(1-Q(\boldsymbol{m}))^{2}
\end{aligned}
$$


With probability at least $1-e^{-c_{0} n}$ for $n \geq n_{0}$ and some $n_{0}, c_{0}>0$, we have $\|\boldsymbol{W}\|_{\text {op }}<3$. On this event, for any $\boldsymbol{m}$ such that $\mathcal{F}(\boldsymbol{m}) \leq-\alpha \lambda^{2} / 2$, we have

$$
M(\boldsymbol{m})^{2}+1 / 2 \cdot(1-Q(\boldsymbol{m}))^{2} \geq \alpha-2 / \lambda^{2}-\|\boldsymbol{W}\|_{\mathrm{op}} / \lambda>\alpha-\varepsilon,
$$

where $\varepsilon=2 / \lambda^{2}+3 / \lambda$. By Cauchy-Schwarz, $Q(\boldsymbol{m}) \geq M(\boldsymbol{m})^{2}$. Then,

$$
Q(\boldsymbol{m})+1 / 2 \cdot(1-Q(\boldsymbol{m}))^{2} \geq \alpha-\varepsilon .
$$

As $2 \alpha-1-2 \varepsilon>0$ for any fixed $\alpha>1 / 2$ and for sufficiently large $\lambda_{0}$, we obtain $Q(\boldsymbol{m}) \geq$ $(2 \alpha-1-2 \varepsilon)^{1 / 2}$. Then, also $(1-Q(\boldsymbol{m}))^{2} \leq\left(1-(2 \alpha-1-2 \varepsilon)^{1 / 2}\right)^{2}$, so

$$
M(\boldsymbol{m})^{2} \geq \alpha-\varepsilon-1 / 2 \cdot(1-Q(\boldsymbol{m}))^{2} \geq(2 \alpha-1-2 \varepsilon)^{1 / 2} .
$$

LEMMA C.2. There exist constants $\lambda_{0}, n_{0}, C_{0}, c_{0}>0$ such that, for any $\lambda \geq \lambda_{0}$ and $n \geq n_{0}$, we have

$$
\mathbb{P}\left(\sup _{\boldsymbol{u} \in \mathrm{B}^{n}(\mathbf{0}, 1)}\left[\frac{1}{n} \sum_{i=1}^{n} \mathbf{1}\left\{\left|\left\langle\boldsymbol{g}_{i}, \boldsymbol{u}\right\rangle\right| \geq \lambda\right\}\right] \geq C_{0} / \lambda^{2}\right) \leq \exp \left\{-c_{0} n\right\},
$$

where $\left\{\boldsymbol{g}_{i}\right\}_{i \in[n]} \stackrel{i . i . d .}{\sim} \mathcal{N}\left(0, \mathbf{I}_{n}\right)$ and $\mathrm{B}^{n}(\mathbf{0}, 1)$ is the $n$-dimensional unit ball centered at $\mathbf{0}$.

ProOF. Let $N(\varepsilon)=\left\{\boldsymbol{v}_{1}, \ldots, \boldsymbol{v}_{|N(\varepsilon)|}\right\}$ be an $\varepsilon$-net of $\mathrm{B}^{n}(\mathbf{0}, 1)$, with $|N(\varepsilon)| \leq(3 / \varepsilon)^{n}$. That is, for any $\boldsymbol{v} \in \mathrm{B}^{n}(\mathbf{0}, 1)$, there exists $\boldsymbol{v}_{\star} \in N(\varepsilon)$, such that $\left\|\boldsymbol{v}-\boldsymbol{v}_{\star}\right\|_{2} \leq \varepsilon$. Then, for any $\boldsymbol{u} \in \mathrm{B}^{n}(\mathbf{0}, 1)$, there exists a sequence $\left\{\boldsymbol{u}_{j}\right\}_{j \geq 0} \subseteq N(\varepsilon)$, such that $\boldsymbol{u}=\sum_{j=0}^{\infty} \varepsilon^{j} \boldsymbol{u}_{j}$. As a consequence, for any vector $g$ we have

$$
\mathbf{1}\{|\langle\boldsymbol{g}, \boldsymbol{u}\rangle| \geq \lambda\} \leq \sum_{j=0}^{\infty} \mathbf{1}\left\{\varepsilon^{j}\left|\left\langle\boldsymbol{g}, \boldsymbol{u}_{j}\right\rangle\right| \geq \lambda / 2^{j+1}\right\},
$$

and hence

$$
\sup _{\boldsymbol{u} \in \mathrm{B}^{n}(\mathbf{0}, 1)} \frac{1}{n} \sum_{i=1}^{n} \mathbf{1}\left\{\left|\left\langle\boldsymbol{g}_{i}, \boldsymbol{u}\right\rangle\right| \geq \lambda\right\} \leq \sum_{j=0}^{\infty} \sup _{\boldsymbol{u} \in N(\varepsilon)} \frac{1}{n} \sum_{i=1}^{n} \mathbf{1}\left\{\left|\left\langle\boldsymbol{g}_{i}, \boldsymbol{u}\right\rangle\right| \geq \lambda /\left[2(2 \varepsilon)^{j}\right]\right\} .
$$

We fix $\varepsilon=1 / 3$ throughout the proof.

First, we show that, for any $\chi \geq 4$ and $\delta \leq 1$, we have (for $\varepsilon=1 / 3$ )

$$
\mathbb{P}\left(\sup _{\boldsymbol{u} \in N(\varepsilon)}\left[\frac{1}{n} \sum_{i=1}^{n} \mathbf{1}\left\{\left|\left\langle\boldsymbol{g}_{i}, \boldsymbol{u}\right\rangle\right| \geq \chi\right\}\right] \geq 16 /\left(\delta \chi^{2}\right)\right) \leq \exp \{-n / \delta\} .
$$

For a fixed $\boldsymbol{u}$ with $\|\boldsymbol{u}\|_{2}=1$, applying the Chernoff-Hoeffding inequality and denoting $\phi(\chi)=\mathbb{P}(|G| \geq \chi)$ for $G \sim \mathcal{N}(0,1)$, we have, for all $t>\phi(\chi)$,

$$
\mathbb{P}\left(\frac{1}{n} \sum_{i=1}^{n} \mathbf{1}\left\{\left|\left\langle\boldsymbol{g}_{i}, \boldsymbol{u}\right\rangle\right| \geq \chi\right\} \geq t\right) \leq \exp \left\{-n \mathrm{D}_{\mathrm{kl}}(t \| \phi(\chi))\right\}
$$

where, for $a, b \in(0,1)$, we define $\mathrm{D}_{\mathrm{kl}}(a \| b)=a \log (a / b)+(1-a) \log ((1-a) /(1-b))$ to be the relative entropy of Bernoulli distribution with parameters $a$ and $b$. Taking union bound over $\boldsymbol{u} \in N(\varepsilon)$ and applying $\phi(\chi) \geq \phi\left(\chi /\|\boldsymbol{u}\|_{2}\right)$,

$$
\begin{aligned}
& \mathbb{P}\left(\sup _{\boldsymbol{u} \in N(\varepsilon)}\left[\frac{1}{n} \sum_{i=1}^{n} \mathbf{1}\left\{\left|\left\langle\boldsymbol{g}_{i}, \boldsymbol{u}\right\rangle\right| \geq \chi\right\}\right] \geq t\right) \\
& \leq \sum_{\boldsymbol{u} \in N(\varepsilon)} \exp \left\{-n \mathrm{D}_{\mathrm{kl}}\left(t \| \phi\left(\chi /\|\boldsymbol{u}\|_{2}\right)\right)\right\} \leq \exp \left\{n\left[-\mathrm{D}_{\mathrm{kl}}(t \| \phi(\chi))+\log (3 / \varepsilon)\right]\right\} .
\end{aligned}
$$


Applying $\varepsilon=1 / 3$ and $\phi(\chi) \leq 2 \exp \left\{-\chi^{2} / 2\right\}$, we have

$$
\begin{aligned}
& -\mathrm{D}_{\mathrm{kl}}(t \| \phi(\chi))+\log (3 / \varepsilon) \\
& \quad=t \log (\phi(\chi))-t \log t+(1-t) \log [1+(t-\phi(\chi)) /(1-t)]+\log 9 \\
& \quad \leq t \log (\phi(\chi))-t \log t+(t-\phi(\chi))+\log 9 \\
& \quad \leq t\left(2-\chi^{2} / 2\right)+3 .
\end{aligned}
$$

Now, take $t=16 /\left(\delta \chi^{2}\right) \geq \phi(\chi)$ for $\chi \geq 4$ and $\delta \leq 1$. Then, $-\mathrm{D}_{\mathrm{kl}}(t \| \phi(\chi))+\log (3 / \varepsilon) \leq$ $-1 / \delta$. This proves equation (C.4). Applying equation (C.4) with $\chi_{j}=\lambda /\left[2(2 \varepsilon)^{j}\right]$ (requiring $\lambda \geq 8$ so that $\left.\chi_{j} \geq 4\right)$ and $\delta_{j}=(2 \varepsilon)^{j} \leq 1$ for $j \geq 0$, we have

$$
\mathbb{P}\left(\sup _{\boldsymbol{u} \in N(\varepsilon)}\left[\frac{1}{n} \sum_{i=1}^{n} \mathbf{1}\left\{\left|\left\langle\boldsymbol{g}_{i}, \boldsymbol{u}\right\rangle\right| \geq \lambda /\left[2(2 \varepsilon)^{j}\right]\right\}\right] \geq 64(2 \varepsilon)^{j} / \lambda^{2}\right) \leq \exp \left\{-n /(2 \varepsilon)^{j}\right\} .
$$

Finally, taking a union bound over $j \geq 0$ and applying

$$
\sum_{j=0}^{\infty} \exp \left\{-n /(2 \varepsilon)^{j}\right\} \leq \exp \left\{-c_{0} n\right\}, \quad \sum_{j=0}^{\infty} 64(2 \varepsilon)^{j} / \lambda^{2} \leq C_{0} / \lambda^{2}
$$

for $n \geq n_{0}$ with some $C_{0}, c_{0}, n_{0}>0$ concludes the proof.

LEMMA C.3. Fix any positive integer $k$. Then, there exist $\lambda_{0}, C_{0}>0$ and functions $c_{0}(\lambda), n_{0}(\lambda)>0$ such that, for all $\lambda \geq \lambda_{0}$ and $n \geq n_{0}(\lambda)$, with probability at least $1-e^{-c_{0}(\lambda) n}$, all points $\boldsymbol{m} \in(-1,1)^{n}$ which satisfy $M(\boldsymbol{m})+Q(\boldsymbol{m}) \geq 1.01$ and $\nabla \mathcal{F}(\boldsymbol{m})=\mathbf{0}$ also satisfy $M(\boldsymbol{m}), Q(\boldsymbol{m}) \geq 1-C_{0} / \lambda^{k}$.

Proof. Since $\boldsymbol{W} \sim \operatorname{GOE}(n)$, we can write $\boldsymbol{W}=\left(\boldsymbol{G}+\boldsymbol{G}^{\boldsymbol{\top}}\right) / \sqrt{2}$, where $\boldsymbol{G}=\left\{G_{i j}\right\}_{i, j \in[n]}$ with $G_{i j} \stackrel{i . i . d .}{\sim} \mathcal{N}(0,1 / n)$. Note, for any $\boldsymbol{m} \in(-1,1)^{n}$, we have $\|\boldsymbol{m}-\mathbf{1}\|_{2} / \sqrt{n} \leq 2$. According to Lemma C.2, there exist constants $C_{0}, c_{0}, \lambda_{0}$ and $n_{0}$ such that, for any $n \geq n_{0}$ and $\lambda \geq \lambda_{0}$, we have

$$
\mathbb{P}\left(\sup _{\boldsymbol{m} \in(-1,1)^{n}}\left[\frac{1}{n} \sum_{i=1}^{n} \mathbf{1}\left\{\left|[\boldsymbol{G}(\boldsymbol{m}-\mathbf{1})]_{i}\right| \geq 0.001 \lambda\right\}\right] \geq C_{0} / \lambda^{2}\right) \leq \exp \left\{-c_{0} n\right\} .
$$

Moreover, by a simple Chernoff bound, we have (denoting $\phi(\lambda)=\mathbb{P}(|G| \geq \lambda) \leq$ $2 \exp \left\{-\lambda^{2} / 2\right\}$ for $\left.G \sim \mathcal{N}(0,1)\right)$

$$
\mathbb{P}\left(\frac{1}{n} \sum_{i=1}^{n} \mathbf{1}\left\{\left|[\boldsymbol{G 1}]_{i}\right| \geq 0.001 \lambda\right\} \geq 3 \phi(0.001 \lambda)\right) \leq \exp \{-n \phi(0.001 \lambda)\} .
$$

The same bounds hold for $\boldsymbol{G}^{\top}$ in place of $\boldsymbol{G}$. For any $\boldsymbol{m} \in(-1,1)^{n}$, note that $\nabla \mathcal{F}(\boldsymbol{m})=\mathbf{0}$ implies

$$
\boldsymbol{m}=\tanh \left(\lambda^{2} M(\boldsymbol{m}) \mathbf{1}+\lambda \cdot \boldsymbol{W} \mathbf{1}+\lambda \cdot \boldsymbol{W}(\boldsymbol{m}-\mathbf{1})-\lambda^{2}[1-Q(\boldsymbol{m})] \boldsymbol{m}\right) .
$$

When the bad events in (C.6) and (C.5) do not happen and $M(\boldsymbol{m})+Q(\boldsymbol{m}) \geq 1.01$, then at least $1-2 C_{0} / \lambda^{2}-6 \phi(0.001 \lambda)$ fraction of coordinates of $\boldsymbol{m}$ satisfy

$$
\begin{aligned}
m_{j} & =\tanh \left(\lambda^{2} M(\boldsymbol{m})+\lambda[\boldsymbol{W} \mathbf{1}]_{j}+\lambda[\boldsymbol{W}(\boldsymbol{m}-\mathbf{1})]_{j}-\lambda^{2}(1-Q(\boldsymbol{m})) m_{j}\right) \\
& \geq \tanh \left((M(\boldsymbol{m})+Q(\boldsymbol{m})-1) \lambda^{2}-0.004 \lambda^{2}\right) \geq \tanh \left(0.006 \lambda^{2}\right) \\
& \geq 1-e^{-0.006 \lambda^{2}}
\end{aligned}
$$


and the remaining coordinates of $\boldsymbol{m}$ satisfy $m_{j} \geq-1$. Therefore, for sufficiently large $\lambda_{0}$, we have

$$
\begin{aligned}
M(\boldsymbol{m}) & =\langle\boldsymbol{m}, \mathbf{1}\rangle / n \\
& \geq\left(1-2 C_{0} / \lambda^{2}-6 \phi(0.001 \lambda)\right)\left(1-e^{-0.006 \lambda^{2}}\right)-2 C_{0} / \lambda^{2}-6 \phi(0.001 \lambda) \\
& \geq 1-6 C_{0} / \lambda^{2} .
\end{aligned}
$$

Hence, we also have

$$
\|\boldsymbol{m}-\mathbf{1}\|_{2} / \sqrt{n} \leq(2-2\langle\boldsymbol{m}, \mathbf{1}\rangle / n)^{1 / 2} \leq\left(12 C_{0}\right)^{1 / 2} / \lambda,
$$

with probability at least $1-e^{-c_{1}(\lambda) n}$ for all $n \geq n_{1}(\lambda)$.

In the following we apply the above argument recursively to prove the lemma. Suppose we already know that $\|\boldsymbol{m}-\mathbf{1}\|_{2} / \sqrt{n} \leq K / \lambda^{k}$ for some constants $K>0$ and $k \geq 1$, with probability $1-e^{-c_{k}(\lambda) n}$ for $n \geq n_{k}(\lambda)$. Applying again Lemma C.2, for $\lambda_{0}$ sufficiently large such that $\varepsilon(\lambda) \equiv K / \lambda^{k} \leq 2$ for all $\lambda \geq \lambda_{0}$, we have with probability at most $\exp \left(-c_{0} n\right)$ that

$$
\sup _{\|\boldsymbol{m}-\mathbf{1}\|_{2} / \sqrt{n} \leq \varepsilon(\lambda)}\left[\frac{1}{n} \sum_{i=1}^{n} \mathbf{1}\left\{\left|[\boldsymbol{G}(\boldsymbol{m}-\mathbf{1})]_{i}\right| \geq 0.001 \lambda\right\}\right] \geq C_{0} \varepsilon(\lambda)^{2} / \lambda^{2},
$$

and similarly for $\boldsymbol{G}^{\top}$. When the bad events in (C.6) and (C.9) do not happen, for any $\boldsymbol{m}$ such that $\|\boldsymbol{m}-\mathbf{1}\|_{2} / \sqrt{n} \leq \varepsilon(\lambda)$, by (C.7) at least $1-2 C_{0} \varepsilon(\lambda)^{2} / \lambda^{2}-6 \phi(0.001 \lambda)$ fraction of coordinates of $\boldsymbol{m}$ satisfy (C.8). Therefore, for sufficiently large $\lambda_{0}$ (depending on $K$ and $k$ ), we have

$$
M(\boldsymbol{m})=\langle\boldsymbol{m}, \mathbf{1}\rangle / n \geq 1-6 C_{0} \varepsilon(\lambda)^{2} / \lambda^{2} .
$$

Hence, we have

$$
\|\boldsymbol{m}-\mathbf{1}\|_{2} / \sqrt{n} \leq(2-2\langle\boldsymbol{m}, \mathbf{1}\rangle / n)^{1 / 2} \leq\left(12 C_{0}\right)^{1 / 2} \varepsilon(\lambda) / \lambda=\left(12 C_{0}\right)^{1 / 2} K / \lambda^{k+1} .
$$

This holds with probability at least $1-e^{-c_{k+1}(\lambda) n}$ for $n \geq n_{k+1}(\lambda)$ for some $0<c_{k+1}(\lambda) \leq$ $c_{k}(\lambda)$ and $n_{k+1}(\lambda) \geq n_{k}(\lambda)$.

Thus, for any fixed $k$, there are $k$-dependent constants $\lambda_{0}, C_{0}>0$ and functions $c_{0}(\lambda)$, $n_{0}(\lambda)$ such that $M(\boldsymbol{m}) \geq 1-C_{0} / \lambda^{k}$ with probability at least $1-e^{-c_{0}(\lambda) n}$ for $\lambda \geq \lambda_{0}$ and $n \geq n_{0}(\lambda)$. Applying $Q(\boldsymbol{m}) \geq M(\boldsymbol{m})^{2}$ by Cauchy-Schwarz, we obtain also the statement for $Q(\boldsymbol{m})$.

Proof of Proposition 5.3. Applying Lemma C. 1 with $\alpha=2 / 3$, for any $\lambda \geq \lambda_{0}$ and $n \geq n_{0}$, with probability at least $1-e^{-c_{0} n}$, all $\boldsymbol{m} \in(-1,1)^{n}$ with $\mathcal{F}(\boldsymbol{m}) \leq-\lambda^{2} / 3$ satisfy $Q(\boldsymbol{m}) \geq M(\boldsymbol{m})^{2} \geq\left(1 / 3-6 / \lambda-4 / \lambda^{2}\right)^{1 / 2}$. For $\lambda_{0}$ large enough and $M(\boldsymbol{m}) \geq 0$, this implies $Q(\boldsymbol{m})+M(\boldsymbol{m}) \geq 1.01$. Hence the proposition holds by Lemma C.3.

\section{APPENDIX D: PROOF OF THEOREM 1.3}

PRoOF OF THEOREM 1.3. Let $\boldsymbol{m}_{\star}$ be any critical point of the TAP free energy $\mathcal{F}=\mathcal{F}_{\lambda, \lambda}$, and let $\boldsymbol{m}$ be any critical point of $\mathcal{F}_{\mathrm{MF}}$. By the conditions $\mathbf{0}=\nabla \mathcal{F}\left(\boldsymbol{m}_{\star}\right)$ and $\mathbf{0}=\nabla \mathcal{F}_{\mathrm{MF}}(\boldsymbol{m})$, we have:

$$
\begin{aligned}
\boldsymbol{m}_{\star} & =\tanh \left(\lambda \cdot \boldsymbol{Y} \boldsymbol{m}_{\star}-\lambda^{2}\left[1-Q\left(\boldsymbol{m}_{\star}\right)\right] \boldsymbol{m}_{\star}\right), \\
\boldsymbol{m} & =\tanh (\lambda \cdot \boldsymbol{Y} \boldsymbol{m}) .
\end{aligned}
$$


For constants $C_{0}, t>0$ to be chosen later, consider the event $\mathcal{E}$ where

$$
\mathcal{E}=\left\{\sup _{\boldsymbol{u} \in \mathrm{B}^{n}(\mathbf{0}, 1)}\left[\frac{1}{n} \sum_{i=1}^{n} \mathbf{1}\left\{\left|(\boldsymbol{W} \boldsymbol{u})_{i}\right| \geq t / \sqrt{n}\right\}\right]<C_{0} / t^{2}\right\}
$$

and $B^{n}(\mathbf{0}, 1)$ denotes the unit ball around $\mathbf{0}$. Note that $\boldsymbol{W}=\left(\boldsymbol{G}+\boldsymbol{G}^{\boldsymbol{\top}}\right) / \sqrt{2 n}$ with $G_{i j} \stackrel{i . i . d}{\sim}$. $\mathcal{N}(0,1)$ for $1 \leq i, j \leq n$, so that

$$
\begin{aligned}
& \sup _{\boldsymbol{u} \in \mathrm{B}^{n}(\mathbf{0}, 1)}\left[\frac{1}{n} \sum_{i=1}^{n} \mathbf{1}\left\{\left|(\boldsymbol{W} \boldsymbol{u})_{i}\right| \geq \frac{t}{\sqrt{n}}\right\}\right] \\
& \leq \sup _{\boldsymbol{u} \in \mathrm{B}^{n}(\mathbf{0}, 1)}\left[\frac{1}{n} \sum_{i=1}^{n} \mathbf{1}\left\{\left|(\boldsymbol{G} \boldsymbol{u})_{i}\right| \geq \frac{t}{\sqrt{2}}\right\}\right]+\sup _{\boldsymbol{u} \in \mathrm{B}^{n}(\mathbf{0}, 1)}\left[\frac{1}{n} \sum_{i=1}^{n} \mathbf{1}\left\{\left|\left(\boldsymbol{G}^{\top} \boldsymbol{u}\right)_{i}\right| \geq \frac{t}{\sqrt{2}}\right\}\right] .
\end{aligned}
$$

Then, by Lemma C.2, for some constant $c_{0}, C_{0}>0$ and for any $t$ sufficiently large, $\mathcal{E}$ holds with probability at least $1-e^{-c_{0} n}$. Define the (random) index set

$$
\mathcal{I}=\left\{i \in\{1, \ldots, n\}:\left|(\boldsymbol{W} \boldsymbol{m})_{i}\right|<t \text { and }\left|\left(\boldsymbol{W} \boldsymbol{m}_{\star}\right)_{i}\right|<t\right\} .
$$

As $\boldsymbol{m} / \sqrt{n} \in \mathrm{B}^{n}(\mathbf{0}, 1)$ and similarly for $\boldsymbol{m}_{\star}$, we have on $\mathcal{E}$ that $|\mathcal{I}| / n \geq 1-2 C_{0} / t^{2}$. Applying $\left|(\boldsymbol{Y} \boldsymbol{m})_{i}\right| \leq|\lambda|+\left|(\boldsymbol{W} \boldsymbol{m})_{i}\right|$ by (1.1), for $i \in \mathcal{I}$ we have

$$
\begin{aligned}
\left|\lambda \cdot\left(\boldsymbol{Y} \boldsymbol{m}_{\star}\right)_{i}-\lambda^{2}\left[1-Q\left(\boldsymbol{m}_{\star}\right)\right] m_{\star, i}\right| & \leq 2 \lambda^{2}+\lambda t, \\
\left|\lambda \cdot(\boldsymbol{Y} \boldsymbol{m})_{i}\right| & \leq \lambda^{2}+\lambda t .
\end{aligned}
$$

Then, taking the difference of (D.1) and (D.2) and applying the lower bound $\tanh ^{\prime}(x) \geq$ $c(\lambda, t)$ for all $|x| \leq 2 \lambda^{2}+\lambda t$ and a constant $c(\lambda, t)>0$, we have

$$
\left|m_{\star, i}-m_{i}\right| \geq c(\lambda, t)\left|\lambda \cdot\left(\boldsymbol{Y} \boldsymbol{m}_{\star}-\boldsymbol{Y} \boldsymbol{m}\right)_{i}-\lambda^{2}\left[1-Q\left(\boldsymbol{m}_{\star}\right)\right] m_{\star, i}\right| .
$$

Then,

$$
\lambda^{4}\left[1-Q\left(\boldsymbol{m}_{\star}\right)\right]^{2} m_{\star, i}^{2} \leq 2 c(\lambda, t)^{-2}\left|m_{\star, i}-m_{i}\right|^{2}+2 \lambda^{2} \cdot\left(\boldsymbol{Y} \boldsymbol{m}_{\star}-\boldsymbol{Y} \boldsymbol{m}\right)_{i}^{2} .
$$

Summing over $i \in\{1, \ldots, n\}$ and using the trivial bound $m_{\star, i}^{2} \leq 1$ for $i \notin \mathcal{I}$, we obtain

$$
\begin{aligned}
\lambda^{4} & {\left[1-Q\left(\boldsymbol{m}_{\star}\right)\right]^{2}\left\|\boldsymbol{m}_{\star}\right\|_{2}^{2} } \\
& \leq \lambda^{4}\left[1-Q\left(\boldsymbol{m}_{\star}\right)\right]^{2}(n-|\mathcal{I}|)+2 c(\lambda, t)^{-2}\left\|\boldsymbol{m}_{\star}-\boldsymbol{m}\right\|_{2}^{2}+2 \lambda^{2}\|\boldsymbol{Y}\|_{\mathrm{op}}^{2}\left\|\boldsymbol{m}_{\star}-\boldsymbol{m}\right\|_{2}^{2} .
\end{aligned}
$$

Suppose $\lambda$ is sufficiently large such that the conclusion $\left|Q\left(\boldsymbol{m}_{\star}\right)-q_{\star}\right|<q_{\star} / 4$ from 5.1 holds with probability $1-\delta / 4$. Choose $t$ large enough such that $2 C_{0} / t^{2}<q_{\star} / 2$, so $|\mathcal{I}| / n>1-$ $q_{\star} / 2$ on the event $\mathcal{E}$. Note also that with probability $1-e^{-c_{0} n}$ for some constant $c_{0}>0$, we have $\|\boldsymbol{Y}\|_{\text {op }} \leq \lambda+3$. Then, on a combined event of probability $1-\delta / 2$, for any critical points $\boldsymbol{m}_{\star}$ and $\boldsymbol{m}$ as above, we obtain

$$
\frac{1}{n}\left\|\boldsymbol{m}_{\star}-\boldsymbol{m}\right\|_{2}^{2} \geq c(\lambda)
$$

for some constant $c(\lambda)>0$.

Consider now any global minimizer $\boldsymbol{m}_{\star}$ of $\mathcal{F}$. Note that $-\boldsymbol{m}_{\star}$ is also a global minimizer of $\mathcal{F}$, so on this combined event, we also have $n^{-1}\left\|\boldsymbol{m}_{\star}+\boldsymbol{m}\right\|_{2}^{2} \geq c(\lambda)$. Then,

$$
\begin{aligned}
\frac{1}{n^{2}}\left\|\boldsymbol{m}_{\star} \boldsymbol{m}_{\star}^{\top}-\boldsymbol{m} \boldsymbol{m}^{\top}\right\|_{F}^{2} & =\frac{1}{n^{2}}\left(\left\|\boldsymbol{m}_{\star}\right\|_{2}^{4}+\|\boldsymbol{m}\|_{2}^{4}-2\left|\left\langle\boldsymbol{m}, \boldsymbol{m}_{\star}\right\rangle\right|^{2}\right) \\
& \geq \frac{1}{2 n^{2}}\left\|\boldsymbol{m}_{\star}-\boldsymbol{m}\right\|_{2}^{2}\left\|\boldsymbol{m}_{\star}+\boldsymbol{m}\right\|_{2}^{2} \geq c(\lambda)^{2} / 2 .
\end{aligned}
$$


On the other hand, note that

$$
\mathcal{F}\left(\boldsymbol{m}_{\star}\right) \leq \mathcal{F}(\boldsymbol{x})=-\frac{\lambda^{2}}{2}-\frac{\lambda}{2 n}\left\langle\boldsymbol{W}, \boldsymbol{x} \boldsymbol{x}^{\top}\right\rangle \leq-\frac{\lambda^{2}}{2}+\lambda,
$$

where the last inequality holds with probability at least $1-e^{-n}$ by the fact that $\left\langle\boldsymbol{W}, \boldsymbol{x} \boldsymbol{x}^{\top}\right\rangle \sim$ $\mathcal{N}(0,2 n)$. For $\lambda$ sufficiently large, we have $-\lambda^{2} / 2+\lambda \leq-\lambda^{2} / 3$. Then, Theorem 1.2 implies, with probability at least $1-\delta / 2$,

$$
\frac{1}{n^{2}}\left\|\boldsymbol{m}_{\star} \boldsymbol{m}_{\star}^{\top}-\widehat{\boldsymbol{X}}_{\text {Bayes }}\right\|_{F}^{2}<c(\lambda)^{2} / 8 .
$$

Combining this with (D.3) and setting $\varepsilon(\lambda)=c(\lambda)^{2} / 8$, with probability at least $1-\delta$, for any critical point $\boldsymbol{m}$ of $\mathcal{F}_{\mathrm{MF}}$ we obtain

$$
\frac{1}{n^{2}}\left\|\boldsymbol{m} \boldsymbol{m}^{\top}-\widehat{\boldsymbol{X}}_{\text {Bayes }}\right\|_{F}^{2} \geq \frac{1}{2 n^{2}}\left\|\boldsymbol{m}_{\star} \boldsymbol{m}_{\star}^{\top}-\boldsymbol{m}^{\top}\right\|_{F}^{2}-\frac{1}{n^{2}}\left\|\boldsymbol{m}_{\star} \boldsymbol{m}_{\star}^{\top}-\widehat{\boldsymbol{X}}_{\text {Bayes }}\right\|_{F}^{2}>\varepsilon(\lambda) .
$$

Acknowledgments. This work was partially supported by grants NSF DMS-1613091, NSF CCF-1714305, NSF IIS-1741162, ONR N00014-18-1-2729, and NSF DMS-1916198. ZF was partially supported by a Hertz Foundation Fellowship. SM was partially supported by Office of Technology Licensing Stanford Graduate Fellowship.

\section{REFERENCES}

[1] AdLeR, R. J. and TAYLOR, J. E. (2007). Random Fields and Geometry. Springer Monographs in Mathematics. Springer, New York. MR2319516

[2] Alaoui, A. E., Krzakala, F. and Jordan, M. I. (2017). Finite size corrections and likelihood ratio fluctuations in the spiked Wigner model. arXiv:1710.02903.

[3] Anderson, G. W., Guionnet, A. and Zeitouni, O. (2010). An Introduction to Random Matrices. Cambridge Studies in Advanced Mathematics 118. Cambridge Univ. Press, Cambridge. MR2760897

[4] Andrieu, C., De Freitas, N., Doucet, A. and Jordan, M. I. (2003). An introduction to MCMC for machine learning. Mach. Learn. 50 5-43.

[5] Auffinger, A. (2011). Random Matrices, Complexity of Spin Glasses and Heavy Tailed Processes. ProQuest LLC, Ann Arbor, MI. Thesis (Ph.D.)-New York University. MR2912239

[6] Auffinger, A. and Ben Arous, G. (2013). Complexity of random smooth functions on the highdimensional sphere. Ann. Probab. 41 4214-4247. MR3161473 https://doi.org/10.1214/13-AOP862

[7] Auffinger, A., Ben Arous, G. and ČERnÝ, J. (2013). Random matrices and complexity of spin glasses. Comm. Pure Appl. Math. 66 165-201. MR2999295 https://doi.org/10.1002/cpa.21422

[8] Auffinger, A. and Jagannath, A. (2019). Thouless-Anderson-Palmer equations for generic $p$-spin glasses. Ann. Probab. 47 2230-2256. MR3980920 https://doi.org/10.1214/18-AOP1307

[9] BAIK, J., BEN Arous, G. and PÉChÉ, S. (2005). Phase transition of the largest eigenvalue for nonnull complex sample covariance matrices. Ann. Probab. 33 1643-1697. MR2165575 https://doi.org/10. 1214/009117905000000233

[10] Bansal, N., Blum, A. and Chawla, S. (2004). Correlation clustering. Mach. Learn. 56 89-113. MR3363423 https://doi.org/10.1023/B:MACH.0000033116.57574.95

[11] BARBIER, J. and MACRIS, N. (2019). The adaptive interpolation method: A simple scheme to prove replica formulas in Bayesian inference. Probab. Theory Related Fields 174 1133-1185. MR3980313 https://doi.org/10.1007/s00440-018-0879-0

[12] Bayati, M. and Montanari, A. (2011). The dynamics of message passing on dense graphs, with applications to compressed sensing. IEEE Trans. Inf. Theory 57 764-785. MR2810285 https://doi.org/10. 1109/TIT.2010.2094817

[13] Ben Arous, G., Mei, S., Montanari, A. and Nica, M. (2019). The landscape of the spiked tensor model. Comm. Pure Appl. Math. 72 2282-2330. MR4011861 https://doi.org/10.1002/cpa.21861

[14] Biane, P. (1997). On the free convolution with a semi-circular distribution. Indiana Univ. Math. J. 46 705718. MR1488333 https://doi.org/10.1512/iumj.1997.46.1467

[15] BLEI, D. M. (2012). Probabilistic topic models. Commun. ACM 55 77-84.

[16] Blei, D. M., KuCuKelbiR, A. and MCAuliffe, J. D. (2017). Variational inference: A review for statisticians. J. Amer. Statist. Assoc. 112 859-877. MR3671776 https://doi.org/10.1080/01621459.2017. 1285773 
[17] Bolthausen, E. (2014). An iterative construction of solutions of the TAP equations for the Sherrington-Kirkpatrick model. Comm. Math. Phys. 325 333-366. MR3147441 https://doi.org/10. 1007/s00220-013-1862-3

[18] Boucheron, S., Lugosi, G. and Massart, P. (2013). Concentration Inequalities: A Nonasymptotic Theory of Independence. Oxford Univ. Press, Oxford. With a foreword by Michel Ledoux. MR3185193 https://doi.org/10.1093/acprof:oso/9780199535255.001.0001

[19] Bray, A. J. and Moore, M. A. (1980). Metastable states in spin glasses. J. Phys. C, Solid State Phys. 13 L469.

[20] Bray, A. J., Moore, M. A. and Young, A. P. (1984). Weighted averages of TAP solutions and Parisi's q (x). J. Phys. C, Solid State Phys. 17 L155.

[21] Brown, L. D. and Purves, R. (1973). Measurable selections of extrema. Ann. Statist. 1 902-912. MR0432846

[22] Capitaine, M., Donati-Martin, C., Féral, D. and Février, M. (2011). Free convolution with a semicircular distribution and eigenvalues of spiked deformations of Wigner matrices. Electron. $J$. Probab. 16 1750-1792. MR2835253 https://doi.org/10.1214/EJP.v16-934

[23] Cavagna, A., Giardina, I., Parisi, G. and Mézard, M. (2003). On the formal equivalence of the TAP and thermodynamic methods in the SK model. J. Phys. A 36 1175-1194. MR1960081 https://doi.org/10.1088/0305-4470/36/5/301

[24] Chatterjee, S. (2010). Spin glasses and Stein's method. Probab. Theory Related Fields 148 567-600. MR2678899 https://doi.org/10.1007/s00440-009-0240-8

[25] Chen, W.-K. and Panchenko, D. (2018). On the TAP free energy in the mixed $p$-spin models. Comm. Math. Phys. 362 219-252. MR3833609 https://doi.org/10.1007/s00220-018-3143-7

[26] Crisanti, A., Leuzzi, L., Parisi, G. and Rizzo, T. (2003). Complexity in the Sherrington-Kirkpatrick model in the annealed approximation. Phys. Rev. B 68174401.

[27] Crisanti, A., Leuzzi, L., Parisi, G. and Rizzo, T. (2004). Spin-glass complexity. Phys. Rev. Lett. 92 127203. https://doi.org/10.1103/PhysRevLett.92.127203

[28] Crisanti, A., Leuzzi, L. and Rizzo, T. (2005). Complexity in mean-field spin-glass models: Ising pspin. Phys. Rev. B 71094202.

[29] Davidson, K. R. and SzAREK, S. J. (2001). Local operator theory, random matrices and Banach spaces. In Handbook of the Geometry of Banach Spaces, Vol. 1131.

[30] De Dominicis, C. and Young, A. P. (1983). Weighted averages and order parameters for the infinite range Ising spin glass. J. Phys. A 16 2063-2075. MR0712998

[31] Dembo, A. and Zeitouni, O. (2010). Large Deviations Techniques and Applications. Stochastic Modelling and Applied Probability 38. Springer, Berlin. Corrected reprint of the second (1998) edition. MR2571413 https://doi.org/10.1007/978-3-642-03311-7

[32] Deshpande, Y., Abbe, E. and Montanari, A. (2017). Asymptotic mutual information for the balanced binary stochastic block model. Inf. Inference 6 125-170. MR3671474 https://doi.org/10.1093/imaiai/ iaw017

[33] Dia, M., Macris, N., Krzakala, F., Lesieur, T., Zdeborová, L. et al. (2016). Mutual information for symmetric rank-one matrix estimation: A proof of the replica formula. In Advances in Neural Information Processing Systems 424-432.

[34] Diaconis, P. (2009). The Markov chain Monte Carlo revolution. Bull. Amer. Math. Soc. (N.S.) 46 179-205. MR2476411 https://doi.org/10.1090/S0273-0979-08-01238-X

[35] Füredi, Z. and KomLÓs, J. (1981). The eigenvalues of random symmetric matrices. Combinatorica 1 233-241. MR0637828 https://doi.org/10.1007/BF02579329

[36] Fyodorov, Y. V. (2004). Complexity of random energy landscapes, glass transition, and absolute value of the spectral determinant of random matrices. Phys. Rev. Lett. 92 240601, 4. MR2115095 https://doi.org/10.1103/PhysRevLett.92.240601

[37] GHorbani, B., JaVADi, H. and MontanaRi, A. (2018). An instability in variational inference for topic models. arXiv: 1802.00568 .

[38] Guerra, F. (2003). Broken replica symmetry bounds in the mean field spin glass model. Comm. Math. Phys. 233 1-12. MR1957729 https://doi.org/10.1007/s00220-002-0773-5

[39] Holland, P. W., Laskey, K. B. and Leinhardt, S. (1983). Stochastic blockmodels: First steps. Soc. Netw. 5 109-137. MR0718088 https://doi.org/10.1016/0378-8733(83)90021-7

[40] Javanmard, A., Montanari, A. and Ricci-Tersenghi, F. (2016). Phase transitions in semidefinite relaxations. Proc. Natl. Acad. Sci. USA 113 E2218-E2223. MR3494080 https://doi.org/10.1073/pnas. 1523097113

[41] Kirkpatrick, S. and Sherrington, D. (1978). Infinite-ranged models of spin-glasses. Phys. Rev. B 17 4384. 
[42] Koller, D. and Friedman, N. (2009). Probabilistic Graphical Models: Principles and Techniques. Adaptive Computation and Machine Learning. MIT Press, Cambridge, MA. MR2778120

[43] Lelarge, M. and Miolane, L. (2019). Fundamental limits of symmetric low-rank matrix estimation. Probab. Theory Related Fields 173 859-929. MR3936148 https://doi.org/10.1007/s00440-018-0845-x

[44] Levin, D. A. and Peres, Y. (2017). Markov Chains and Mixing Times. Amer. Math. Soc., Providence, RI. Second edition of [MR2466937], With contributions by Elizabeth L. Wilmer, With a chapter on "Coupling from the past" by James G. Propp and David B. Wilson. MR3726904 https://doi.org/10. 1090/mbk/107

[45] Mézard, M. and Montanari, A. (2009). Information, Physics, and Computation. Oxford Graduate Texts. Oxford Univ. Press, Oxford. MR2518205 https://doi.org/10.1093/acprof:oso/9780198570837. 001.0001

[46] Mézard, M., Parisi, G. and Virasoro, M. A. (1987). Spin Glass Theory and Beyond. World Scientific Lecture Notes in Physics 9. World Scientific Co., Inc., Teaneck, NJ. MR1026102

[47] Miolane, L. (2017). Fundamental limits of low-rank matrix estimation: the non-symmetric case. arXiv:1702.00473.

[48] Mityagin, B. S. (2020). The Zero Set of a Real Analytic Function. Mat. Zametki $107473-475$. MR4070868 https://doi.org/10.4213/mzm12620

[49] Montanari, A. and SEn, S. (2016). Semidefinite programs on sparse random graphs and their application to community detection. In STOC'16-Proceedings of the 48th Annual ACM SIGACT Symposium on Theory of Computing 814-827. ACM, New York. MR3536616 https://doi.org/10.1145/2897518. 2897548

[50] Montanari, A. and Venkataramanan, R. (2020). Estimation of low-rank matrices via approximate message passing. Ann. Statist. To appear. arXiv:1711.01682.

[51] Nishimori, H. (2001). Statistical Physics of Spin Glasses and Information Processing: An Introduction. International Series of Monographs on Physics 111. Oxford Univ. Press, New York. Translated from the 1999 Japanese original. MR2250384 https://doi.org/10.1093/acprof:oso/9780198509417.001.0001

[52] PARISI, G. (1979). Infinite number of order parameters for spin-glasses. Phys. Rev. Lett. 431754.

[53] PARISI, G. (1980). A sequence of approximated solutions to the SK model for spin glasses. J. Phys. A: Math. Gen. 13 L115.

[54] Parisi, G. (1983). Order parameter for spin-glasses. Phys. Rev. Lett. 50 1946-1948. MR0702601 https://doi.org/10.1103/PhysRevLett.50.1946

[55] PARisi, G. and PotTers, M. (1995). On the number of metastable states in spin glasses. Europhys. Lett. 3213.

[56] PAStUR, L. A. (1972). The spectrum of random matrices. Teoret. Mat. Fiz. 10 102-112. MR0475502

[57] PAStUR, L. A. (2005). A simple approach to the global regime of Gaussian ensembles of random matrices. Ukraïn. Mat. Zh. 57 790-817. MR2208456 https://doi.org/10.1007/s11253-005-0241-4

[58] Perry, A., Wein, A. S., Bandeira, A. S. and Moitra, A. (2018). Optimality and sub-optimality of PCA I: Spiked random matrix models. Ann. Statist. 46 2416-2451. MR3845022 https://doi.org/10. 1214/17-AOS1625

[59] PlefKa, T. (1982). Convergence condition of the TAP equation for the infinite-ranged Ising spin glass model. J. Phys. A 15 1971-1978. MR0663708

[60] Sinclair, A. (2012). Algorithms for Random Generation and Counting: A Markov Chain Approach. Springer Science \& Business Media.

[61] Singer, A. (2011). Angular synchronization by eigenvectors and semidefinite programming. Appl. Comput. Harmon. Anal. 30 20-36. MR2737931 https://doi.org/10.1016/j.acha.2010.02.001

[62] Singer, A. and Wu, H. (2011). Orientability and diffusion maps. Appl. Comput. Harmon. Anal. 31 44-58. MR2795874 https://doi.org/10.1016/j.acha.2010.10.001

[63] Subag, E. (2017). The complexity of spherical $p$-spin models-a second moment approach. Ann. Probab. 45 3385-3450. MR3706746 https://doi.org/10.1214/16-AOP1139

[64] Talagrand, M. (2011). Mean Field Models for Spin Glasses. Volume I: Basic Examples. Ergebnisse der Mathematik und Ihrer Grenzgebiete. 3. Folge. A Series of Modern Surveys in Mathematics [Results in Mathematics and Related Areas. 3rd Series. A Series of Modern Surveys in Mathematics] 54. Springer, Berlin. MR2731561 https://doi.org/10.1007/978-3-642-15202-3

[65] Thouless, D. J., Anderson, P. W. and Palmer, R. G. (1977). Solution of 'solvable model of a spin glass'. Philos. Mag. 35 593-601.

[66] Voiculescu, D. (1991). Limit laws for random matrices and free products. Invent. Math. 104 201-220. MR1094052 https://doi.org/10.1007/BF01245072

[67] Wainwright, M. J. and JordAn, M. I. (2008). Graphical models, exponential families, and variational inference. Found. Trends Mach. Learn. 1 1-305.

[68] ZHANG, A. Y. and ZHOU, H. H. (2017). Theoretical and computational guarantees of mean field variational inference for community detection. arXiv:1710.11268. 